\title{
ANÁLISE DE ONDALETAS EM SÉRIES TEMPORAIS
}

\author{
Chang Chiann
}

\section{TESE APRESENTADA}

$\mathrm{AO}$

INSTITUTO DE MATEMÁTICA E ESTATÍSTICA

DA UNIVERSIDADE DE SÃO PAULO

PARA OBTENÇÃO DO GRAU DE DOUTOR

EM

ESTATÍSTICA

Área de Concentração: Estatística

Orientador: Prof. Dr. Pedro Alberto Morettin

Este trabalho foi financiado pelo $\mathrm{CNPq}$

- São Paulo, Junho de 1997 - 


\section{ANÁLISE DE ONDALETAS EM SÉRIES TEMPORAIS}

Este exemplar corresponde à redação final

da tese devidamente corrigida, apresentada por Chang Chiann e aprovada pela Comissão Julgadora.

São Paulo, 31 de Julho de 1997

\section{Banca Examinadora}

Prof. Dr. Pedro Alberto Morettin (Presidente) IME-USP Prof. Dra. Clélia Maria de Castro Toloi IME-USP

Prof. Dr. David R. Brillinger

Prof. Dr. Luiz Koodi Hotta

Prof. Dr. Ronaldo Dias
UNIV. CALIFORNIA IMECC-UNICAMP IMECC-UNICAMP 
A minha família Anil e Edwin 


\section{Agradecimentos}

Graças a Deus por sua presença constante, que me deu forças e saúde para a realização deste trabalho. E também graças a Ele, contei com a ajuda de muitas pessoas, em particular, queremos agradecer:

Ao Prof. Dr. Pedro A. Morettin, orientador, amigo e como um pai, pelo carinho, compreensão, paciência e dedicação com que transmitiu seus conhecimentos.

À Profa. Dra. Clélia M. C. Toloi, grande amiga, pela amizade, pelas sugestões e pelo apoio e incentivo constante.

Aos Prof. Dr. David R. Brillinger, Prof. Dr. Rainer von Sachs e Prof. Dr. Rainer Dahlhaus, pelas sugestões e críticas apresentadas.

Aos Prof. David S. Stoffer e Prof. Miguel A. Arino, pelo fornecimento de dados.

A minha família, pelo apoio e compreensão. 


\section{Summary}

In this work we use wavelets to analyze stationary and locally stationary processes. In the case of stationary processes we introduce a time-scale analysis and in the case of locally stationary processes we develop a method to estimate the coefficients of a time-varying linear system.

The concept of wavelet spectrum of a stationary process is introduced and asymptotic properties of the discrete wavelet transform are derived. The wavelet periodogram is defined as an estimator of the wavelet spectrum and its properties studied. For the time-varying linear system, a wavelet expansion for the coefficients gives a procedure for the estimation of those filter coefficients and properties are derived. Applications to real data are given. 


\section{Sumário}

1 Introdução 3

2 Ondaletas 6

2.1 Introdução . . . . . . . . . . . . . . . . . 6

2.2 Análise de Fourier e de Ondaletas . . . . . . . . . . . . . . 6

2.3 A Transformada de Ondaletas . . . . . . . . . . . . . 12

2.4 Reconstrução de Funções . . . . . . . . . . . . . . . . . . . . . 14

2.5 A Transformada Discreta de Ondaletas . . . . . . . . . 15

2.6 Análise de Multiresolução . . . . . . . . . . . . . . . . . . 17

2.7 Decomposição e Reconstrução de Ondaletas ...... . 20

2.8 Encolhimento de Ondaletas . . . . . . . . . . . . . . . . 27

3 Processos Estacionários e Não-Estacionários 30

3.1 Introdução . . . . . . . . . . . . . . . . . . . . . 30

3.2 Processos Estacionários . . . . . . . . . . . . . . . . . 31

3.3 Processos Não-Estacionários . . . . . . . . . . . . . 35

3.4 Processos Localmente Estacionários . . . . . . . . . . . . . 41

4 Análise Espectral via Ondaletas 45

4.1 Introdução . . . . . . . . . . . . . . . . . . . . 45 
4.2 O Espectro de Ondaletas . . . . . . . . . . . . . . . . . 46

4.3 A Transformada Finita de Ondaletas e Suas Propriedades Assintóticas . . . . . . . . . . . . . . . . 51

4.4 O Periodograma de Ondaletas . . . . . . . . . . . . 57

4.5 O Escalograma ...................... 62

4.6 Encolhimento de Ondaletas . . . . . . . . . . . . 65

4.7 Aplicação . . . . . . . . . . . . . . . . . . . . 66

5 Estimação de Sistemas Lineares Variando no Tempo Utilizando Ondaletas

5.1 Introdução . . . . . . . . . . . . . . . . . . . . . 69

5.2 Bases de Ondaletas Ortonormais e Estimadores de Ondaletas do Espectro Dependente do Tempo . . . . . . . . . . . . 70

5.3 Sistemas Lineares Variando no Tempo . . . . . . . . . . . 73

5.4 Aplicação . . . . . . . . . . . . . . . . . . 85

$\begin{array}{ll}\text { Discussões e Conclusões } & 86\end{array}$

$\begin{array}{ll}\text { Referências } & 87\end{array}$

Apêndice A: Funções de Ondaletas Ortogonais 94

Apêndice B: Figuras Citadas no Capítulo 4 101

Apêndice C: Figuras Citadas no Capítulo 5 103 


\section{Capítulo 1}

\section{Introdução}

A análise de Fourier é uma ferramenta eficaz e bem conhecida para a análise de séries temporais. Depois da publicação do influente trabalho de Blackman e Tukey (1959) e da introdução do algoritmo chamado transformada rápida de Fourier - FF' por Cooley e Tukey (1965), a área de análise espectral de séries temporais experimentou um progresso contínuo. Referências são Brillinger (1975) e Brockwell e Davis (1991) para a parte teórica e Percival e Walden (1993) para a parte de aplicação. É conhecido que a análise de Fourier é bem adequada para analisar séries temporais estacionárias. Para as séries temporais categóricas, uma ferramenta adequada é a análise de Walsh-Fourier e para detalhes, ver Morettin (1981) e Stoffer (1991).

Recentemente, uma nova técnica chamada de ondaleta foi introduzida em diversas áreas, tais como processamento e codificação de sinais e imagens, compressão de dados e imagens, análise numérica, estudo de turbulências, geofísica, estatística, etc, ver Mallat (1989), Grossman e Morlet (1984), Meyer (1990). Em particular, essa técnica é util para lidar com as séries temporais não estacionárias e com os sinais contaminados com ruídos. Isto se deve ao fato de que as funções de ondaleta são localizadas no tempo, ou seja, elas nos permitem analisar uma série no tempo e escala simultaneamente, ao contrário das funções de Fourier que são localizadas apenas nas frequências e não no tempo. Os livros de Daubechies (1992) e Chui (1992) são boas referências matemáticas. Para uma introdução elementar de ondaletas, ver Strang (1989) e Farge (1992).

A teoria de ondaletas inspirou o desenvolvimento de uma metodologia 
poderosa para processar sinais, imagens e outros tipos de dados científicos. Tal metodologia inclue diversas ferramentas, tais como a transformada de ondaletas, análise de multiresolução, análise de tempo-escala, representação de tempo-frequência com bases de ondaletas, etc. $\mathrm{O}$ estudo de ondaletas como uma nova disciplina começou na década de oitenta, mas a sua história está apenas começando. A pesquisa nesta área está progredindo com alta velocidade, novas técnicas importantes se apresentam a cada ano, expandindo rapidamente o domínio de aplicação das ondaletas.

$\mathrm{Na}$ análise de Fourier, o sistema ortonormal das funções exponenciais complexas $\left\{w_{n}(x)=e^{i n x}, n=0, \pm 1, \cdots\right\}$ são obtidas pela dilatação da função $w(x)=e^{i x}$ através de $w_{n}(x)=w(n x)$. Portanto, cada função periódica com período $2 \pi$, que pertence ao $L^{2}(0,2 \pi)$, é gerada pela superposição de $w_{n}(x)$. Estendemos essa idéia para o espaço $L^{2}(\mathcal{R})$, isto é, a partir de uma função denominada $\psi$ geramos as funções de ondaletas, pela translação e dilatação de $\psi$, através da relação

$$
\psi_{j, k}(x)=2^{-j / 2} \psi\left(2^{-j} x-k\right), \quad j, k \in Z,
$$

onde $Z=\{0, \pm 1, \cdots\}$. Estas funções geradas formam uma base ortonormal em $L^{2}(\mathcal{R})$, para algumas escolhas de $\psi$.

Do ponto de vista da estatística, ondaletas são utilizadas na estimação de densidades (Hall e Patil, 1993a,b, Donoho, 1993, Donoho et al, 1996), na análise de regressão não-paramétrica (Nason, 1995, Hall e Patil, 1993c, Donoho e Johnstone, 1995a,b, Donoho et al, 1995) e na estimação da densidade espectral (Gao, 1993, 1997, Moulin, 1994, Neumann, 1996). Brillinger (1994, 1996) utilizou ondaletas para detectar a mudança de níveis em séries hidrológicas e desenvolveu um método de estimação para as funções do nível médio baseado em ondaletas. Nason e Silverman (1994) desenvolveram um software para calcular os coeficientes das transformadas discretas de ondaleta. Este algoritmo requer apenas $O(N)$ operações que é mais rápido que o de FFT, cujo número de operações é de $O\left(N \log _{2} N\right)$. Para séries longas, a economia de tempo é considerável. Bruce e Gao (1996) apresentaram algumas aplicações de análise de ondaleta através de um software chamado S+WAVELETS. Na análise de processos não-estacionários, as referências são Dahlhaus (1996, 1997), von Sachs e Schneider (1996), Neumann e von Sachs (1997) e Priestley (1996).

No Capítulo 2, abordamos alguns tópicos fundamentais de ondaletas, incluindo a transformada de ondaletas, a transformada discreta de ondaletas, a 
aproximação de ondaletas, análise de multiresolução, decomposição e reconstrução de ondaletas e o método de encolhimento.

No Capítulo 3, apresentamos os conceitos necessários sobre processos estacionários, não-estacionários e localmente estacionários.

No Capítulo 4, desenvolvemos uma análise espectral de ondaletas para um processo estacionário discreto. As propriedades assintóticas das transformadas discretas de ondaleta de uma amostra de valores observados desse processo são obtidas e o periodograma de ondaleta é considerado como um estimador do espectro de ondaleta. Apresentamos uma aplicação para uma série real.

No Capítulo 5 analisamos sistemas lineares cujos coeficientes variam no tempo. O objetivo é estimar estas coeficientes e para isso utilizamos expansões dos mesmos em ondaletas. Uma aplicação para dados reais é apresentada. 


\section{Capítulo 2}

\section{Ondaletas}

\subsection{Introdução}

Recentemente, as ondaletas tornaram-se muito populares em diversas áreas científicas, podendo ser vistas como novas bases para representar funções ou, ainda, constituindo-se numa nova técnica para a análise de funções no domínio de tempo-frequência. As ondaletas são uma ferramenta polivalente com rico conteúdo matemático e grande potencial de aplicação.

\subsection{Análise de Fourier e de Ondaletas}

Seja $L^{2}(0,2 \pi)$ a coleção de todas as funções mensuráveis definidas no intervalo $(0,2 \pi) \mathrm{com}$

$$
\int_{0}^{2 \pi}|f(x)|^{2} d x<\infty
$$

Geralmente, as funções que pertencem a $L^{2}(0,2 \pi)$ são estendidas periodicamente para $\mathcal{R}=(-\infty, \infty)$, isto é, $f(x)=f(x-2 \pi)$ para todo $x$. Qualquer função $f$ de $L^{2}(0,2 \pi)$ pode ser decomposta em termos de uma série de Fourier, 


$$
f(x)=\sum_{n=-\infty}^{\infty} C_{n} e^{i n x},
$$

onde as constantes $C_{n}$ são os coeficientes de Fourier de $f$ e são definidos por

$$
C_{n}=\frac{1}{2 \pi} \int_{0}^{2 \pi} f(x) e^{-i n x} d x .
$$

Notamos que $f$ é decomposta numa soma de infinitos componentes ortogonais $g_{n}(x)=C_{n} e^{i n x}$, onde ortogonalidade significa

$$
<g_{m}, g_{n}>=0, \quad m \neq n,
$$

com $<g_{m}, g_{n}>=\frac{1}{2 \pi} \int_{0}^{2 \pi} g_{m}(x) \overline{g_{n}(x)} d x$.

A ortogonalidade de $g_{n}(x)$ se deve ao fato de que

$$
w_{n}(x)=e^{i n x}, \quad n=0, \pm 1, \cdots,
$$

é uma base ortonormal de $L^{2}(0,2 \pi)$ e essa base é gerada por dilatações da função

$$
w(x)=e^{i x}
$$

através de

$$
w_{n}(x)=w(n x) .
$$

Portanto, cada função periódica com período $2 \pi$, que pertence a $L^{2}(0,2 \pi)$, é gerada pela superposição de $w_{n}(x)$.

Pela propriedade de ortonormalidade de $\left\{w_{n}\right\}$, a série de Fourier (2.1) satisfaz a relação de Parseval

$$
\frac{1}{2 \pi} \int_{0}^{2 \pi}|f(x)|^{2}=\sum_{n=-\infty}^{\infty}\left|C_{n}\right|^{2},
$$

$\operatorname{com} \sum_{n=-\infty}^{\infty}\left|C_{n}\right|^{2}<\infty$.

Vimos, então, que a função 


$$
w(x)=e^{i x}=\cos x+i \sin x
$$

(onda senoidal) é a única função necessária para gerar todas as funções de $L^{2}(0,2 \pi)$, portanto cada função de $L^{2}(0,2 \pi)$ é composta de uma superposição de ondas com frequências diferentes.

Agora, consideremos o espaço $L^{2}(\mathcal{R})$ das funções mensuráveis $f$ definidas em $\mathcal{R}=(-\infty, \infty)$ e que satisfaz

$$
\int_{-\infty}^{\infty}|f(x)|^{2} d x<\infty
$$

Estendemos a idéia de que a função $w(x)=e^{i x}$ gera $L^{2}(0,2 \pi)$ para o espaço $L^{2}(\mathcal{R})$, isto é, a partir de uma função chamada $\psi$ geramos todas as funções de $L^{2}(\mathcal{R})$, por translações e dilatações de $\psi$, através da relação

$$
\psi_{a, b}(x)=|a|^{-\frac{1}{2}} \psi\left(\frac{x-b}{a}\right), \quad a, b \in \mathcal{R}, \quad a \neq 0
$$

Podemos notar que cada função em $L^{2}(\mathcal{R})$ decai para zero nos pontos $\pm \infty$, o que não acontece com as funções senoidais $w_{n}$. Por isso, as ondas que geram $L^{2}(\mathcal{R})$ também devem decair para zero, nos pontos $\pm \infty$, rapidamente. Portanto, essas ondas devem ser pequenas, ou seja, ondaletas.

Existem ondaletas "pai" $\phi$ e ondaletas "mãe" $\psi$ tais que,

$$
\int \phi(t) d t=1, \quad \int \psi(t) d t=0 .
$$

Geralmente, as ondaletas "pai" $\phi$ representam bem as partes suaves e de baixa frequência de um sinal e as ondaletas "mãe" $\psi$ representam bem as partes detalhes e de alta frequência de um sinal.

Existem muitas famílias diferentes formadas por pares de ondaletas "pai" e "mãe". Exceto em alguns casos especiais, não existe forma analítica para calcular uma função ondaleta. Para avaliar uma função ondaleta, usamos a equação de dilatação. que é o âmago da análise de ondaletas. Para uma ondaleta "pai" $\phi(x)$, a equação de dilatação é definida por

$$
\phi(x)=\sqrt{2} \sum_{k} h(k) \phi(2 x-k),
$$


e a ondaleta "mãe" $\psi(x)$ pode ser obtida através de

$$
\psi(x)=\sqrt{2} \sum_{k} g(k) \phi(2 x-k),
$$

onde os coeficientes $h(k)$ e $g(k)$ são filtros "low pass" e "high pass", respectivamente. Para detalhes, ver seção 2.6.

Para algumas escolhas especiais de $\psi, a$ e $b$, as funções $\psi_{a, b}(x)$ constituem uma base ortonormal para $L^{2}(\mathcal{R})$. Em particular, se escolhermos $a=2^{j}$, $b=2^{j} k, j, k \in \mathcal{Z}$, então existem funções $\psi$, tal que

$$
\psi_{j, k}(x)=\psi_{a, b}(x)=2^{-\frac{2}{2}} \psi\left(2^{-j} x-k\right), \quad j, k \in \mathcal{Z},
$$

constituem uma base ortonormal para $L^{2}(\mathcal{R})$.

Definição 2.1: A função $\psi \in L^{2}(\mathcal{R})$ é chamada ondaleta ortonormal se a família $\left\{\psi_{j, k}\right\}$, como definida no (2.6) é uma base ortonormal de $L^{2}(\mathcal{R})$, isto é,

$$
<\psi_{j, k}, \psi_{l, m}>=\delta_{j, l} \delta_{k, m}, \quad j, k, l, m \in \mathcal{Z},
$$

onde

$$
\delta_{j, k}= \begin{cases}1, & \text { se } j=k \\ 0, & \text { se } j \neq k\end{cases}
$$

e cada função $f \in L^{2}(\mathcal{R})$ pode ser escrita em termos de uma série de ondaleta

$$
f(x)=\sum_{j, k=-\infty}^{\infty} c_{j, k} \psi_{j, k}(x) .
$$

Analogamente à notação dos coeficientes de Fourier (2.2), os coeficientes de ondaleta $c_{j, k}$ são dados por

$$
c_{j, k}=<f, \psi_{j, k}>
$$

As ondaletas que constituem uma base ortonormal de $L^{2}(\mathcal{R})$ são as funções que satisfazem certos requerimentos, tais como:

(i) $\int_{-\infty}^{\infty} \psi(x) d x=0$; 
(ii) as funções $\psi_{j, k}(x), j, k \in \mathcal{Z}$ são ortonormais,

$$
\int_{-\infty}^{\infty} \psi_{j_{1}, k_{1}}(x) \psi_{j_{2}, k_{2}}(x) d x=\delta_{j_{1}, j_{2}} \delta_{k_{1}, k_{2}}, \quad\left(j_{1}, k_{1}\right) \neq\left(j_{2}, k_{2}\right) ;
$$

(iii) os primeiros $(r-1)$ momentos de $\psi$ são nulos,

$$
\int x^{j} \psi(x) d x=0
$$

para algum $r \geq 1, j=0,1, \cdots, r-1 \mathrm{e}$

$$
\int\left|x^{r} \psi(x)\right| d x<\infty
$$

Existem várias famílias de ondaletas, sendo que as mais utilizadas na prática tem suporte compacto. As ondaletas ortogonais mais utilizadas em geral são:

Haar : a ondaleta de Haar é uma onda quadrática, foi introduzida pelo matemático Haar em 1910 e forneceu a primeira representação em série de ondaleta ortogonal. A ondaleta de Haar tem suporte compacto; é a única ondaleta ortogonal compacta, mas não é contínua. Além disso, possui uma forma analítica:

$$
\psi(x)=\left\{\begin{aligned}
1, & 0 \leq x<1 / 2 \\
-1, & 1 / 2 \leq x<1 \\
0, & \text { caso contrário }
\end{aligned}\right.
$$

$\mathrm{e}$

$$
\phi(x)= \begin{cases}1, & 0 \leq x<1 \\ 0, & \text { caso contrário. }\end{cases}
$$

Daublets : as ondaletas "Daublets" foram o primeiro tipo de ondaletas ortogonais contínuas com suporte compacto. Esse tipo de ondaletas foi introduzido por Ingrid Daubechies.

Symmlets : as ondaletas "Symmlets" também têm suporte compacto e também foram construidas por Daubechies. As "Daublets" são totalmente assimétricas e as "Symmlets" foram construidas para serem as mais simétricas possiveis. 
Coiflets : as ondaletas "Coiflets" também foram construidas por Daubechies para serem quase simétricas e possuem propriedades adicionais (momentos nulos para $\phi$ e $\psi$ ).

Os gráficos dessas ondaletas estão no Apêndice A.

A escolha de uma ondaleta necessita de um compromisso entre diferentes propriedades tais como suavização, localização espacial, localização de frequência, habilidade para representar funções polinomiais locais, ortogonalidade e simetria. Essas propriedades são discutidas a seguir:

Suavização: a suavização da aproximação de ondaletas é uma das propriedades da análise de ondaletas. Para muitas aplicações, a função ondaleta deve ser suficientemente suave para representar eficientemente as características de um sinal. A falta de suavização é uma das principais desvantagens da ondaleta de Haar.

Uma medida de suavização para uma ondaleta é dada pelo número de derivadas da ondaleta. A ondaleta de Haar é discontínua e portanto não é diferenciavel em todo ponto.

Localização espacial: uma característica principal da análise de ondaletas é a habilidade de localizar características de um sinal no tempo e no espaço. O tamanho do suporte de uma ondaleta é relacionado com esta habilidade. Ondaletas muito compactas, tais como a ondaleta de Haar, são muito bem localizadas no tempo e no espaço. Geralmente o tamanho do suporte é relacionado com a suavização, as ondaletas mais suaves têm suportes mais largos.

Momentos nulos: uma ondaleta com um número alto de momentos nulos pode representar melhor os sinais polinomiais de grau mais alto. Os números de momentos nulos são também relacionados com a suavização de uma ondaleta. Quanto maior o número de momentos nulos que essa ondaleta tem, mais suave ela é.

Localização de frequência: ondaletas localizam características de um sinal não somente no tempo e no espaço, mas também na frequência. Em geral, ondaletas mais suaves têm melhores propriedades de localização de frequência. 
Simetria: com exceção da ondaleta de Haar, as ondaletas ortogonais que têm suportes compactos são não simétricas. As "Daublets" são altamente não simétricas e as "Symmlets" e "Coiflets" são bastante simétricas.

Ortogonalidade: a ortogonalidade das transformadas de ondaletas é uma característica principal para algumas aplicações.

\subsection{A Transformada de Ondaletas}

$\mathrm{Na}$ análise de Fourier, a transformada de Fourier $\mathcal{F}$, definida por

$$
(\mathcal{F} f)(w)=\int_{-\infty}^{\infty} e^{-i w x} f(x) d x, \quad f \in L^{2}(\mathcal{R})
$$

não só é uma ferramenta matemática poderosa, como também tem interpretações físicas significantes em aplicações. Se uma função $f \in L^{2}(\mathcal{R})$ é considerada como um sinal, definimos a "energia" dessa função como sendo sua norma

$$
\|f\|_{2}=\int|f(t)|^{2} d t
$$

Segue-se que a transformada de Fourier

$$
\hat{f}(w)=(\mathcal{F} f)(w)
$$

de $f$ representa o espectro desse sinal. Na análise de sinais, estes são definidos no domínio de tempo e as informações espectrais desses sinais são dadas no domínio de frequência. Uma identidade fundamental conhecida como a identidade de Parseval descreve a relação entre as funções em $L^{2}(\mathcal{R})$ e suas transformadas de Fourier e é dada por

$$
<f, g>=\frac{1}{2 \pi}<\hat{f}, \hat{g}>, \quad f, g \in L^{2}(\mathcal{R})
$$

Consequentemente, notamos que a "energia" do sinal $f$ é diretamente proporcional ao seu espectro,

$$
\|f\|_{2}=\frac{1}{2 \pi}\|\hat{f}\|_{2}, \quad f \in L^{2}(\mathcal{R}) .
$$

Esta relação é conhecida como conservação de energia. 
Entretanto, notamos que as informações espectrais $\hat{f}(w)$ de $f(t)$, na frequência $w$, não são bem localizadas no tempo, isto é, essas informações são obtidas por $f$ usando suas informações passadas e futuras, e assim, se um sinal é alterado numa pequena vizinhança de um instante $t$, todas as informações espectrais podem ser afetadas. Como a frequência de um sinal é inversamente proporcional ao tamanho de seu ciclo, o ideal seria usar um intervalo de tempo pequeno para obter os fenômenos de alta frequência com maior precisão e um intervalo de tempo largo para analizar o comportamento em baixa frequência com maior precisão. Isto é, é importante ter uma janela de tempo-frequência flexível que automaticamente se estreita nas altas frequências e se amplia nas baixas frequências. Mas na análise de Fourier, a janela de tempo-frequência é rígida e seu tamanho é fixo em observar qualquer frequência, portanto essa análise não é adequada para analisar os sinais com frequências muito altas e baixas.

A transformada de ondaletas $w_{\psi}$, em $L^{2}(\mathcal{R})$, com respeito a uma base de ondaleta $\psi$, definida por

$$
\left(w_{\psi} f\right)(b, a)=|a|^{-\frac{1}{2}} \int_{-\infty}^{\infty} f(t) \psi\left(\frac{t-b}{a}\right) d t, \quad f \in L^{2}(\mathcal{R}), \quad a, b \in \mathcal{R}, \quad a \neq 0,
$$

fornece uma janela de tempo-frequência flexível no qual automaticamente se estreita quando observamos o comportamento do sinal em alta frequência (com pequeno valor de $a$ ) e se amplia quando estudamos o comportamento em baixa frequência (com grande valor de $a$ )

A relação de Parseval também vale para ondaletas, isto é,

$$
\frac{1}{C_{\psi}} \iint\left|w_{\psi} f(a, b)\right|^{2} d a d b=\int|f(t)|^{2} d t
$$

onde

$$
C_{\psi}=\int_{-\infty}^{\infty} \frac{|\hat{\psi}(w)|^{2}}{|w|} d w<\infty
$$

e $\hat{\psi}(w)$ é a transformada de Fourier de $\psi$ na frequência $w$.

Podemos escrever

$$
E=\frac{1}{C_{\psi}} \iint\left|w_{\psi} f(a, b)\right|^{2} d a d b
$$

como a "energia total" de $f$. Então a energia também se conserva na transformada de ondaletas. 


\subsection{Reconstrução de Funções}

Cada função $f \in L^{2}(\mathcal{R})$ pode ser reconstruida pela transformada de ondaletas. Agora vamos estudar quatro diferentes situações para reconstrução: $\mathcal{R}$;

(a) Reconstrução de $f \in L^{2}(\mathcal{R})$ baseada em $\left(w_{\psi} f\right)(b, a)$ com $a, b \in$

Neste caso, a função $f$ é reconstruida por

$$
f(x)=\frac{1}{C_{\psi}} \iint_{\mathcal{R}^{2}}\left\{\left(w_{\psi} f\right)(b, a)\right\} \psi_{a, b}(x) \frac{d a d b}{a^{2}},
$$

com $\psi$ satisfazendo a condição de admissibilidade:

$$
C_{\psi}=\int_{-\infty}^{\infty} \frac{|\hat{\psi}(w)|^{2}}{|w|} d w<\infty .
$$

(b) Reconstrução de $f \in L^{2}(\mathcal{R})$ baseada em $\left(w_{\psi} f\right)(b, a)$ com $b \in \mathcal{R}$ e $a>0$;

$\mathrm{Na}$ análise de tempo-frequência, usamos uma constante positiva, múltipla de $a^{-1}$ para representar frequência, portanto, como somente as frequências positivas são de interesse, precisamos reconstruir a função $f$ baseada numa classe menor de $\psi$ que satisfaz

$$
\int_{0}^{\infty} \frac{|\hat{\psi}(w)|^{2}}{w} d w=\int_{0}^{\infty} \frac{|\hat{\psi}(-w)|^{2}}{w} d w=\frac{1}{2} C_{\psi}<\infty
$$

e $f$ é reconstruida por

$$
f(x)=\frac{2}{C_{\psi}} \int_{0}^{\infty}\left\{\int_{-\infty}^{\infty}\left[\left(w_{\psi} f\right)(b, a)\right] \psi_{a, b}(x) d b\right\} \frac{d a}{a^{2}} .
$$

Neste caso, comparando com (2.15), a base de ondaleta $\psi$ é mais restrita.

(c) Reconstrução de $f \in L^{2}(\mathcal{R})$ baseada em $\left(w_{\psi} f\right)(b, a) \operatorname{com} b \in \mathcal{R}$ e $a=2^{j}, j \in \mathcal{Z}$;

Tomando $a=2^{j}, f$ pode ser reconstruída por

$$
f(x)=\sum_{j=-\infty}^{\infty} \int_{-\infty}^{\infty}\left\{2^{-\frac{2}{2}}\left(w_{\psi} f\right)\left(b, 2^{j}\right)\right\}\left\{2^{-j} \psi\left(2^{-j}(x-b)\right)\right\} d b,
$$


onde $\psi$ satisfaz a condição de estabilidade:

$$
A \leq \sum_{j=-\infty}^{\infty}\left|\hat{\psi}\left(2^{j} w\right)\right|^{2} \leq B
$$

onde $A$ e $B$, com $0<A \leq B<\infty$, são constantes que independem de $w$. Isto implica que $\psi$ também satisfaz

$$
A \ln 2 \leq \int_{0}^{\infty} \frac{|\hat{\psi}(w)|^{2}}{w} d w, \quad \int_{0}^{\infty} \frac{|\hat{\psi}(-w)|^{2}}{w} d w \leq B \ln 2
$$

ou seja, $2 A \ln 2 \leq C_{\psi} \leq 2 B \ln 2$.

(d) Reconstrução de $f \in L^{2}(\mathcal{R})$ baseada em $\left(w_{\psi} f\right)(b, a) \operatorname{com} b=2^{j} k$ e $a=2^{j}, j, k \in \mathcal{Z}$;

Numa base ortogonal de ondaleta, tomamos $a=2^{j}$ e $b=2^{j} k$, então temos

$$
\begin{aligned}
c_{j, k} & =\left(w_{\psi} f\right)\left(2^{j} k, 2^{j}\right) \\
& =\int_{-\infty}^{\infty} f(x) 2^{-\frac{1}{2}} \psi\left(2^{-j} x-k\right) d x \\
& =<f, \psi_{j, k}>
\end{aligned}
$$

e a função $f$ pode ser reconstruída por

$$
f(x)=\sum_{j, k=-\infty}^{\infty}<f, \psi_{j, k}>\psi_{j, k}(x),
$$

onde $\psi_{j, k}(x)=2^{-\frac{2}{2}} \psi\left(2^{-j} x-k\right)$.

\subsection{A Transformada Discreta de Ondaletas}

A aproximação de série de ondaletas ortogonais para um sinal $f(t)$ em tempo contínuo é dada por

$f(t) \approx \sum_{k} s_{J, k} \phi_{j, k}(t)+\sum_{k} d_{J, k} \psi_{J, k}(t)+\sum_{k} d_{J-1, k} \psi_{J-1, k}(t)+\cdots+\sum_{k} d_{1, k} \psi_{1, k}(t)$,

onde $J$ é o número de componentes de multiresolução e $k$ assume os valores de 1 até o número de coeficientes no componente correspondente. Os coeficientes 
$s_{J, k}, d_{J, k}, \cdots, d_{1, k}$ são os coeficientes da transformada de ondaletas. As funções $\phi_{J, k}(t)$ e $\psi_{j, k}(t)$ são as funções ondaletas ortogonais, isto é,

$$
\begin{gathered}
\int \phi_{J, k}(t) \phi_{J, k^{\prime}}(t) d t=\delta_{k, k^{\prime}}, \\
\int \psi_{j, k}(t) \phi_{J, k^{\prime}}(t)=0, \\
\int \psi_{j, k}(t) \psi_{j^{\prime}, k^{\prime}}(t)=\delta_{j, j^{\prime}} \delta_{k, k^{\prime}} .
\end{gathered}
$$

As funções $\phi_{J, k}(t)$ e $\psi_{j, k}(t)$ são geradas a partir de $\phi$ e $\psi$, por translação e dilatação,

$$
\phi_{J, k}(t)=2^{-J / 2} \phi\left(2^{-J} t-k\right),
$$

e

$$
\psi_{j, k}(t)=2^{-j / 2} \psi\left(2^{-j} t-k\right) .
$$

Os coeficientes de ondaletas são obtidos por

$$
s_{J, k} \approx \int \phi_{J, k}(t) f(t) d t
$$

e

$$
d_{j, k} \approx \int \psi_{j, k}(t) f(t) d t, \quad j=1,2, \cdots, J
$$

A transformada discreta de ondaletas (DWT) calcula os coeficientes de ondaletas $s_{J, k}, d_{J, k}, \cdots, d_{1, k}$ para um sinal discreto $f_{1}, f_{2}, \cdots, f_{n}$ de dimensão finita. A DWT transforma o vetor $f=\left(f_{1}, \cdots, f_{n}\right)^{\prime}$ num vetor de $n$ coeficientes de ondaletas $w_{\psi}=\left(w_{1}, \cdots, w_{n}\right)^{\prime}$.

$\mathrm{O}$ vetor $w_{\psi}$ contém os coeficientes de ondaletas $s_{J, k}$ e $d_{j, k}, j=1,2, \cdots, J$. Os coeficientes $s_{J, k}$ são chamados coeficientes suaves e os coeficientes $d_{j, k}$ são chamados coeficientes de detalhes. Os $s_{J, k}$ representam o comportamento suave dos dados na escala $2^{J}$ e os $d_{J, k}$ fornecem o comportamento detalhado na escala $2^{J}$ e $d_{J-1, k}, \cdots, d_{1, k}$ representam progressivamente o comportamento detalhado nas escalas mais finas.

A DWT é matematicamente equivalente a multiplicação por uma matriz ortogonal $W$, isto é,

$$
w_{\psi}=W f
$$

$\mathrm{Na}$ prática, para calcular os coeficientes da transformada discreta de ondaletas, não fazemos essa multiplicação, usamos uma algoritmo piramidal que 
requer apenas $O(n)$ operações, que é mais rápido que o da transformada de Fourier (FFT) cujo número de operações é de $O\left(n \log _{2} n\right)$. Para detalhes, ver seção 2.7 .

\subsection{Análise de Multiresolução}

Uma análise de multiresolução consiste numa sequência crescente de subespaços fechados $V_{j}, j \rightarrow-\infty$, que aproximam $L^{2}(\mathcal{R})$. Mais precisamente:

$$
\cdots V_{1} \subset V_{0} \subset V_{-1} \subset \cdots
$$

$$
\overline{\cup_{j \in \mathcal{Z}} V_{j}}=L^{2}(\mathcal{R})
$$

(3)

$$
\cap_{j \in \mathcal{Z}} V_{j}=\{\emptyset\}
$$

(4)

$$
f(x) \in V_{j} \Longleftrightarrow f(2 x) \in V_{j-1} \Longleftrightarrow f\left(2^{j} x\right) \in V_{0}, \quad j \in \mathcal{Z} .
$$

Se denotamos por $P_{j}$ a projeção ortogonal sobre $V_{j}$, então (2.24) garante que

$$
\lim _{j \rightarrow-\infty} P_{j} f=f, \quad f \in L^{2}(\mathcal{R})
$$

Um exemplo de espaço $V_{j}$ que satisfaz (2.23)-(2.26) é a análise de multiresolução de Haar:

$$
V_{j}=\left\{f \in L^{2}(\mathcal{R}) ; \forall k \in \mathcal{Z}: f_{\left[2^{\jmath} k, 2^{\jmath}(k+1)[\right.}=\text { constante }\right\} .
$$

Outra propriedade da análise multiresolução é a invariância de $V_{0}$ sobre translação inteira, isto é,

$$
f \in V_{0} \Longleftrightarrow f(\cdot-k) \in V_{0}, \quad k \in \mathcal{Z} .
$$


Agora, suponhamos que exista uma função $\phi$ (função escala). $\phi \in V_{0}$, tal que

$$
\left\{\phi_{0, k}: k \in \mathcal{Z}\right\}
$$

é uma base ortonormal em $V_{0}$, onde, para todo $j, k \in \mathcal{Z}$,

$$
\phi_{j, k}(x)=2^{-\frac{j}{2}} \phi\left(2^{-j} x-k\right) .
$$

(2.26) e (2.28) implicam que $\left\{\phi_{j, k} ; k \in \mathcal{Z}\right\}$ é uma base ortonormal para $V_{j}$ para todo $j \in \mathcal{Z}$.

O princípio básico da análise de multiresolução é que quando uma coleção de subespaços fechados satisfazem (2.23)-(2.28), então existe uma base de ondaletas ortonormal $\left\{\psi_{j, k} ; j, k \in \mathcal{Z}\right\}$ de $L^{2}(\mathcal{R})$,

$$
\psi_{j, k}(x)=2^{-\frac{1}{2}} \psi\left(2^{-j} x-k\right),
$$

tal que, para todo $f \in L^{2}(\mathcal{R})$,

$$
P_{j-1} f=P_{j} f+\sum_{k \in \mathcal{Z}}<f, \psi_{j, k}>\psi_{j, k},
$$

onde $P_{j}$ é a projeção ortogonal em $V_{j}$ e $\left\langle f, \psi_{j, k}>\right.$ são os coeficientes de ondaleta. Além disso, a ondaleta $\psi$ pode ser construída explicitamente.

Para cada $j \in \mathcal{Z}$, definimos $W_{j}$ como o complemento ortogonal de $V_{j}$ em $V_{j-1}$ :

$$
V_{j-1}=V_{j} \oplus W_{j}
$$

e

$$
W_{j} \perp W_{j^{\prime}} \text { se } j \neq j^{\prime} .
$$

Então, para $j<J$, temos

$$
V_{j}=V_{J} \oplus_{k=0}^{J-j-1} W_{J-k},
$$

onde todos esses subespaços são ortogonais. Em virtude de (2.24) e (2.25), temos

$$
L^{2}(\mathcal{R})=\oplus_{j \in \mathcal{Z}} W_{j}
$$

uma decomposição de $L^{2}(\mathcal{R})$ em subespaços mutualmente ortogonais. Além disso, os espaços $W_{j}$ herdam a propriedade (2.26) de $V_{j}$ :

$$
f \in W_{j} \Longleftrightarrow f\left(2^{j} \cdot\right) \in W_{0} .
$$


Por (2.29), temos que, para um $j$ fixo, $\left\{\psi_{j, k} ; k \in \mathcal{Z}\right\}$ constitui uma base ortonormal para $W_{j}$. Logo, a coleção $\left\{\psi_{j, k} ; j, k \in \mathcal{Z}\right\}$ é uma base ortonormal para $L^{2}(\mathcal{R})$.

Como $\phi \in V_{0} \subset V_{-1}$ e $\left\{\phi_{-1, k}\right\}$ é uma base ortonormal em $V_{-1}$, então qualquer função em $V_{0}$ pode ser escrita como uma combinação linear das funções básicas $\phi_{-1, k}$ de $V_{-1}$. Em particular, temos

$$
\phi(x)=\sum_{n} h(n) \phi_{-1, n}=\sum_{n} h(n) \sqrt{2} \phi(2 x-n),
$$

$\operatorname{com} h(n)=<\phi, \phi_{-1, n}>=\sqrt{2} \int \phi(x) \phi(2 x-n) d x$ e $\sum_{n \in \mathcal{Z}}|h(n)|^{2}=1$.

Como $\psi \in W_{0}$ é equivalente ao $\psi \in V_{-1}$ e $\psi \perp V_{0}$, uma possibilidade para a construção da ondaleta $\psi$ é

$$
\psi=\sum_{n} g(n) \phi_{-1, n},
$$

com $g(n)=(-1)^{n} h(-n+1)$, ou seja,

$$
\psi(x)=\sqrt{2} \sum_{n}(-1)^{n} h(-n+1) \phi(2 x-n) .
$$

Para a análise de multiresolução de Haar, temos

$$
\phi(x)= \begin{cases}1, & 0 \leq x<1 \\ 0, & \text { caso contrário. }\end{cases}
$$

Portanto,

$$
h(n)=\left\{\begin{aligned}
\frac{1}{\sqrt{2}}, & \text { se } n=0,1 \\
0, & \text { caso contrário. }
\end{aligned}\right.
$$

Consequentemente,

$$
\psi=\frac{1}{\sqrt{2}} \phi_{-1,0}-\frac{1}{\sqrt{2}} \phi_{-1,1}
$$

ou seja,

$$
\psi(x)=\left\{\begin{aligned}
1, & 0 \leq x<1 / 2 \\
-1, & 1 / 2 \leq x<1 \\
0, & \text { caso contrário }
\end{aligned}\right.
$$


Em termos da aproximação de ondaletas, pela (2.32), podemos decompor um sinal $f(t)$ como uma soma dos componentes ortogonais

$$
S_{J}(t), D_{J}(t), D_{J-1}(t), \cdots, D_{1}(t)
$$

em diferentes escalas,

$$
f(t) \approx S_{J}(t)+D_{J}(t)+\cdots+D_{1}(t),
$$

onde as funções

$$
S_{J}(t)=\sum_{k} s_{J, k} \phi_{J, k}(t)
$$

$\mathrm{e}$

$$
D_{j}(t)=\sum_{k} d_{j, k} \psi_{j, k}(t)
$$

representam os sinais suaves e detalhes, respectivamente e os $s_{J, k}$ e $d_{j, k}$ são definidos em (2.22). Como esses termos nas diferentes escalas representam os componentes do sinal $f(t)$ nas diferentes resoluções, essa aproximação é chamada uma decomposição de multiresolução.

\subsection{Decomposição e Reconstrução de Onda- letas}

A análise de multiresolução conduz naturalmente a um esquema hierárquico e rápido para o cálculo dos coeficientes de ondaletas de uma dada função. Suponha que conhecemos $\left\langle f, \phi_{j, k}\right\rangle$ em alguma escala fina (sem perda de generalidade, assumamos que para essa escala $j=0$ ), então é fácil calcular os coeficientes de ondaletas $\left\langle f, \psi_{j, k}>\right.$ para $j \geq 1$.

Como vimos anteriormente, temos

$$
\psi=\sum_{n} g(n) \phi_{-1, n}
$$

onde $g(n)=<\psi, \phi_{-1, n}>=(-1)^{n} h(-n+1)$, consequentemente,

$$
\begin{aligned}
\psi_{j, k}(x) & =2^{-\frac{2}{2}} \psi\left(2^{-j} x-k\right) \\
& =2^{-\frac{2}{2}} \sum_{n} g(n) 2^{1 / 2} \phi\left(2^{-j+1} x-2 k-n\right)
\end{aligned}
$$




$$
\begin{aligned}
& =\sum_{n} g(n) \phi_{j-1,2 k+n}(x) \\
& =\sum_{n} g(n-2 k) \phi_{j-1, n}(x) .
\end{aligned}
$$

Portanto,

$$
<f, \psi_{1, k}>=\sum_{n} \overline{g(n-2 k)}<f, \phi_{0, n}>.
$$

Analogamente, temos

$$
<f, \psi_{j, k}>=\sum_{n} \overline{g(n-2 k)}<f, \phi_{j-1, n}>
$$

e por $(2.35)$, temos

$$
\phi_{j, k}(x)=\sum_{n} h(n-2 k) \phi_{j-1, n}(x) .
$$

Portanto,

$$
<f, \phi_{j, k}>=\sum_{n} \overline{h(n-2 k)}<f, \phi_{j-1, n}>\text {. }
$$

Resumindo o procedimento,

(1) a partir $\operatorname{dos}\left\langle f, \phi_{0, n}\right\rangle$, calculamos os $\left\langle f, \psi_{1, k}\right\rangle$ usando (2.37) e os $<f, \phi_{1, k}>$ usando (2.39);

(2) repetir o item (1) para calcular os $\left\langle f, \psi_{2, k}\right\rangle,\left\langle f, \phi_{2, k}\right\rangle$ a partir de $<f, \phi_{1, n}>$

e assim recursivamente. Em cada etapa, calculamos não somente os coeficientes de ondaleta $\left\langle f, \psi_{j, k}\right\rangle$ do correspondente nível $j$, mas também os $\left.<f, \phi_{j, k}\right\rangle$ para o mesmo nível $j$, os quais são usados para o cálculo dos coeficientes de ondaletas do próximo nível.

Pela propriedade (2.24), cada função $f \in L^{2}(\mathcal{R})$ pode ser aproximada por uma $f_{J} \in V_{J}$, para algum $J \in \mathcal{Z}$. Começamos com uma escala fina de aproximação para $f$,

$$
f^{0}=P_{0} f
$$

onde $P_{j}$ é a projeção ortogonal em $V_{j}$ e denotamos $Q_{j}$ a projeção ortogonal em $W_{j}$. Agora decompomos

$$
f^{0} \in V_{0}=V_{1} \oplus W_{1}
$$


em

$$
f^{0}=f^{1}+\delta^{1}
$$

onde

$$
f^{1}=P_{1} f^{0}=P_{1} f
$$

é a aproximação seguinte de $f$ na análise de multiresolução e

$$
\delta^{1}=f^{0}-f^{1}=Q_{1} f^{0}=Q_{1} f
$$

é a perda na transição $f^{0} \rightarrow f^{1}$.

Em cada desses espaços $V_{j}, W_{j}$, temos bases ortonormais $\left\{\phi_{j, k} ; k \in \mathcal{Z}\right\}$, $\left\{\psi_{j, k} ; k \in \mathcal{Z}\right\}$, respectivamente, então

$$
f^{0}=\sum_{n} c_{0, n} \phi_{0, n}, \quad f^{1}=\sum_{n} c_{1, n} \phi_{1, n}, \quad \delta^{1}=\sum_{n} d_{1, n} \psi_{1, n},
$$

onde

$$
c_{j, n}=<f, \phi_{j, n}>, \quad d_{j, n}=<f, \psi_{j, n}>.
$$

E por (2.37) e (2.39), temos

$$
c_{1, k}=\sum_{n} \overline{h(n-2 k)} c_{0, n}, \quad d_{1, k}=\sum_{n} \overline{g(n-2 k)} c_{0, n} .
$$

Para uma sequência $a=\left\{a_{n} ; n \in \mathcal{Z}\right\}, \bar{a}=\left\{\overline{a_{-n}} ; n \in \mathcal{Z}\right\}$, os operadores $H$ e $G$ são definidos como

$$
(H a)_{k}=\sum_{n} h(2 k-n) a_{n}
$$

e

$$
(G a)_{k}=\sum_{n} g(2 k-n) a_{n} .
$$

Esses operadores são conhecidos como os filtros "low pass" e "high pass", respectivamente. Podemos reescrever $(2.40)$ como

$$
C_{1}=\bar{H} C_{0}, \quad D_{1}=\bar{G} C_{0},
$$

onde $C_{j}=\left(c_{j, 1}, \cdots, c_{j, n}\right)^{\prime}$ e $D_{j}=\left(d_{j, 1}, \cdots, d_{j, n_{\jmath}}\right)^{\prime}$ e $n_{j}$ é o número de componentes no nivel $j$.

A aproximação

$$
f^{1} \in V_{1}=V_{2} \oplus W_{2}
$$


pode novamente ser decomposta em

$$
f^{1}=f^{2}+\delta^{2}, \quad f^{2} \in V_{2}, \quad \delta^{2} \in W_{2},
$$

com

$$
f^{2}=\sum_{n} c_{2, n} \phi_{2, n}, \quad \delta^{2}=\sum_{n} d_{2, n} \psi_{2, n}
$$

Assim, temos

$$
C_{2}=\bar{H} C_{1}, \quad D_{2}=\bar{G} C_{1} .
$$

Esquematizando o processo, temos

$$
\begin{array}{rllllll}
C_{0} & \stackrel{\bar{H}}{\longrightarrow} & C_{1} & \stackrel{\bar{H}}{\longrightarrow} & C_{2} & \cdots & C_{J} \\
& \searrow \bar{G} & D_{1} & \searrow \bar{G} & D_{2} & \ddots & D_{J}
\end{array}
$$

Isto é, reescrevemos a informação $\left(<f, \phi_{0, n}>\right)_{n \in Z}=C_{0}$ em termos de $D_{1}, D_{2}, \cdots, D_{J}$ e $C_{J}$, ou seja, em termos de $\left(<f, \psi_{j, k}>\right)_{k \in \mathcal{Z}, j=1, \cdots, J}$ e $(<$ $\left.f, \phi_{J, k}>\right)_{k \in \mathcal{Z}}$. Esse processo é conhecido como o algoritmo piramidal.

Para um sinal discreto $f_{1}, \cdots, f_{n}$, a entrada $C_{0}=\left(c_{0,1}, \cdots, c_{0, n}\right)^{\prime}$ consiste nos valores desse sinal:

$$
c_{0, i}=f_{i}, \quad i=1,2, \cdots, n .
$$

Se $n_{j}=\frac{n}{2^{j}}$, a saída do algoritmo é o conjunto de coeficientes de detalhes da transformada discreta de ondaletas (DWT)

$$
D_{j}=\left(d_{j, 1}, \cdots, d_{j, n_{j}}\right)^{\prime}
$$

nos níveis $j=1,2, \cdots, J$, o qual correspondente às escalas $2,4,8, \cdots, 2^{J}$, junto com os coeficientes suaves da DWT

$$
C_{J}=\left(c_{J, 1}, \cdots, c_{J, n_{J}}\right)^{\prime} .
$$

Como os filtros $H$ e $G$ têm um número fixo dé coeficientes, o algoritmo requer $O(n)$ operações.

Como todas as transformações são feitas em bases ortogonais, a operação inversa é dada pelas matrizes adjuntas. Explicitamente,

$$
\begin{aligned}
f^{j-1} & =f^{j}+\delta^{j} \\
& =\sum_{k} c_{j, k} \phi_{j, k}+\sum_{k} d_{j, k} \psi_{j, k}
\end{aligned}
$$


Portanto,

$$
\begin{aligned}
c_{j-1, n} & =<f^{j-1}, \phi_{j-1, n}> \\
& =\sum_{k} c_{j, k}<\phi_{j, k}, \phi_{j-1, n}>+\sum_{k} d_{j, k}<\psi_{j, k}, \phi_{j-1, n}> \\
& =\sum_{k}\left\{h(n-2 k) c_{j, k}+g(n-2 k) d_{j, k}\right\} \\
& =H^{*} C_{j}+G^{*} D_{j},
\end{aligned}
$$

onde os operadores $H^{*}$ e $G^{*}$ são definidos como

$$
\left(H^{*} a\right)_{n}=\sum_{k} h(n-2 k) a_{k}
$$

e

$$
\left(G^{*} a\right)_{n}=\sum_{k} g(n-2 k) a_{k} .
$$

Logo, o algoritmo de reconstrução é dado por

$$
\begin{aligned}
& C_{0} \quad \stackrel{H^{*}}{\longleftarrow} \quad C_{1} \quad \stackrel{H^{*}}{\longleftarrow} \quad C_{2} \quad \cdots \quad C_{J} \\
& \nwarrow G^{*} D_{1} \quad G^{*} D_{2} \quad \ddots \quad D_{J}
\end{aligned}
$$

Esse algoritmo também requer $O(n)$ operações.

Para a ondaleta de Haar, temos

$$
\phi(x)=\phi(2 x)+\phi(2 x-1),
$$

e

$$
\psi(x)=\phi(2 x)-\phi(2 x-1),
$$

e portanto os coeficientes são:

$$
h(0)=h(1)=\frac{1}{\sqrt{2}}, \quad g(0)=g(-1)=\frac{1}{\sqrt{2}}
$$

Exemplo 2.1: Dado um vetor de dados

$$
Y=(1,0,-3,2,1,0,1,2) .
$$

A decomposição de ondaleta desses dados é 


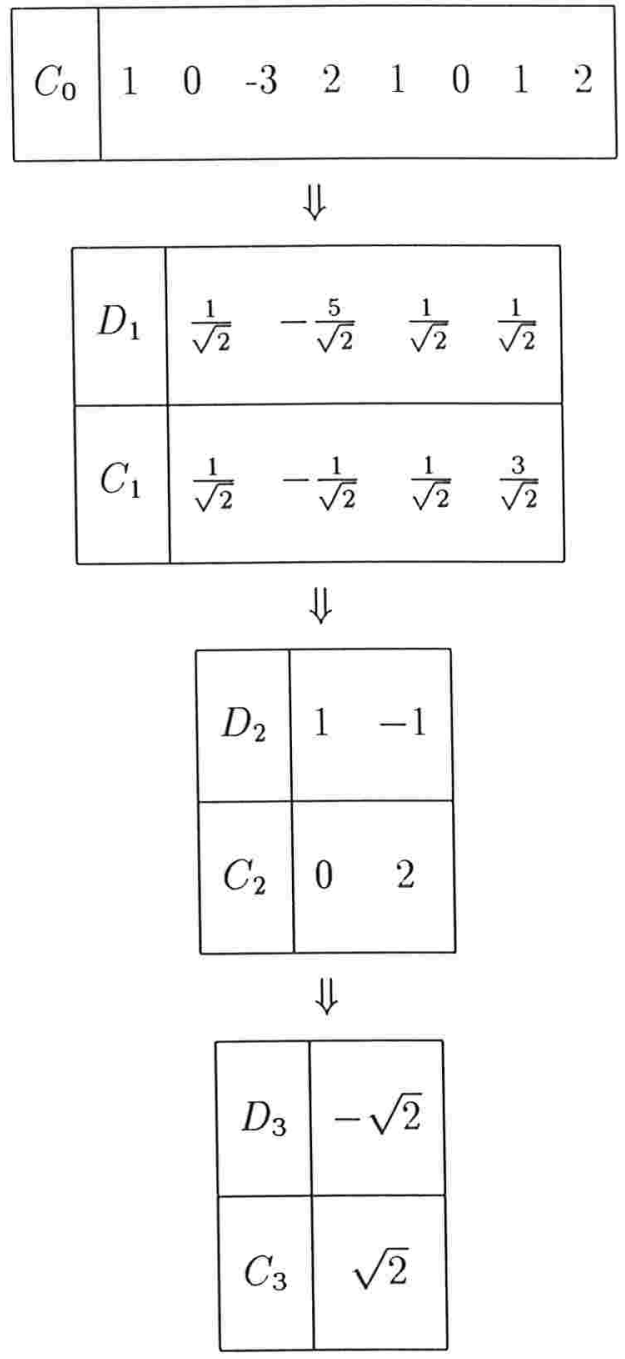

E o processo de reconstrução é dado como 


\begin{tabular}{|c|c|c|c|c|c|c|c|}
\hline & & $C_{3}$ & $\sqrt{2}$ & ${ }^{*} \rightarrow$ & 1 & 1 & \\
\hline & & $D_{3}$ & $-\sqrt{2}-$ & $\rightarrow$ & -1 & 1 & \\
\hline & & $C_{2}$ & & & 0 & 2 & \\
\hline & & & $\swarrow$ & & & & \\
\hline$C_{2}$ & 0 & 2 & $-H^{*} \rightarrow$ & 0 & 0 & $\frac{2}{\sqrt{2}}$ & $\frac{2}{\sqrt{2}}$ \\
\hline$D_{2}$ & & -1 & $-G^{*} \rightarrow$ & $\frac{1}{\sqrt{2}}$ & $-\frac{1}{\sqrt{2}}$ & $-\frac{1}{\sqrt{2}}$ & $\frac{1}{\sqrt{2}}$ \\
\hline$C_{1}$ & & & & $\frac{1}{\sqrt{2}}$ & $-\frac{1}{\sqrt{2}}$ & $\frac{1}{\sqrt{2}}$ & $\frac{3}{\sqrt{2}}$ \\
\hline
\end{tabular}

\begin{tabular}{|l|cccc|c|ccccccccc|}
\hline$C_{1}$ & $\frac{1}{\sqrt{2}}$ & $-\frac{1}{\sqrt{2}}$ & $\frac{1}{\sqrt{2}}$ & $\frac{3}{\sqrt{2}}$ & $-H^{*} \rightarrow$ & $\frac{1}{2}$ & $\frac{1}{2}$ & $-\frac{1}{2}$ & $-\frac{1}{2}$ & $\frac{1}{2}$ & $\frac{1}{2}$ & $\frac{3}{2}$ & $\frac{3}{2}$ \\
\hline$D_{1}$ & $\frac{1}{\sqrt{2}}$ & $-\frac{5}{\sqrt{2}}$ & $\frac{1}{\sqrt{2}}$ & $-\frac{1}{\sqrt{2}}$ & $-G^{*} \rightarrow$ & $\frac{1}{2}$ & $-\frac{1}{2}$ & $-\frac{5}{2}$ & $\frac{5}{2}$ & $\frac{1}{2}$ & $-\frac{1}{2}$ & $-\frac{1}{2}$ & $\frac{1}{2}$ \\
\hline \hline$C_{0}$ & & & & & & 1 & 0 & -3 & 2 & 1 & 0 & 1 & 2 \\
\hline
\end{tabular}




\subsection{Encolhimento de Ondaletas}

Nos problemas de regressão não-paramétrica, queremos estimar um sinal desconhecido $f(t)$ a partir de alguns dados $y_{i}$. Suponha que temos o modelo

$$
y_{i}=f_{i}+\epsilon_{i}, \quad i=1, \cdots, n
$$

onde $f_{i}$ é um sinal discreto e $\epsilon_{i}$ são erros normais, independentes e identicamente distribuidos, $\epsilon_{i} \sim N\left(0, \sigma^{2}\right)$. A regressão paramétrica usual precisa de um modelo particular para $f_{i}$ e na regressão não-paramétrica fazemos algumas suposições mínimas sobre a natureza exata de $f_{i}$. Donoho e co-autores (Donoho, 1993, Donoho e Johnstone, 1990, 1994, Donoho, Johnstone, Kerkyacharian e Picard, 1995, Donoho, 1995 e Donoho e Johnstone, 1995b) desenvolveram uma metodologia interessante para regressão não-paramétrica e suavização baseada no princípio de encolhimento de ondaletas, que se refere a remover os valores pequenos dos coeficientes de ondaletas que estão tendendo a zero, os quais são considerados como os ruídos no processo.

O encolhimento de ondaletas se refere a estimadores obtidos nas seguintes etapas:

1. aplicar a transformada discreta de ondaletas;

2. truncar os coeficientes de ondaletas pequenos (tendendo a zero);

3. aplicar a transformada discreta inversa de ondaletas.

O encolhimento de ondaletas é capaz de remover os ruídos de um sinal enquanto preserva as características desse sinal. A metodologia original de Donoho e co-autores focaliza em erros Gaussianos independentes e identicamente distribuidos. Essa metodologia foi estendida para uma classe maior de modelos e situações, tais como as situações com ruídos correlacionados (Johnstone e Silverman, 1994, Neumann e von Sachs, 1995), estimação de densidade de probabilidade (Johnstone, Kerkyacharian e Picard, 1992, Delyon e Juditsky, 1993, Tribouley, 1995, Vannucci e Vidakovic, 1995), estimação de densidade espectral (Moulin, 1992, 1994, Gao, 1993, 1997, Percival, 1993, von Sachs e Schneider, 1996, Neumann e von Sachs, 1995, Walden, McCoy e Percival, 1995), problemas de inversão (Donoho, 1991, Miller e Willsky, 1994, Xia, Kuo e Suter, 1994, Kolaczyk, 1994, Lina e Mayrand, 1995), análise discriminante e 
de classificação (Saito e Coifman, 1994, Buckheit e Donoho, 1995), análise fatorial (Wickerhauser, 1994) e problema de mudança de pontos (Wang, 1995).

A estimação usando encolhimento de ondaletas se baseia em três princípios:

1. as características de um sinal podem ser representadas somente por poucos coeficientes de ondaletas;

2. os ruídos afetam todos os coeficientes de ondaletas;

3. truncando os coeficientes de ondaletas que estão tendendo a zero, os ruídos podem ser removidos e as características de sinal são preservadas.

O processo de encolhimento de ondaletas se resume em três etapas:

1. aplicar a transformada de ondaletas com $J$ níveis para um sinal $Y$, obtendo os coeficientes de ondaletas $D_{1}, \cdots, D_{J}, C_{J}$;

2. truncar os coeficientes, nas escalas mais finas $j, D_{1}, \cdots, D_{j}$, para obter novos coeficientes

$$
\tilde{D}_{1}=\delta_{\lambda_{1} \sigma_{1}}\left(D_{1}\right), \cdots, \tilde{D}_{j}=\delta_{\lambda_{j} \sigma_{j}}\left(D_{j}\right) .
$$

A função $\delta_{c}(x)$ trunca $x$ que tende a zero e é parametrizada por $c=\lambda \sigma$, onde $\lambda$ é o valor do truncamento e $\sigma^{2}$ é a variância amostral dos ruídos;

3. aplicar a transformada inversa de ondaletas usando os coeficientes

$$
\tilde{D}_{1}, \cdots, \tilde{D}_{j}, D_{j+1}, \cdots, D_{J}
$$

para obter as estimativas de $\hat{f}$.

Os resultados teóricos mostram que para certa escolha de $\lambda_{j}$, o estimador $\hat{f}_{\text {ws }}$ de encolhimento de ondaletas pode alcançar o risco minimax sobre uma classe ampla de funções $\mathcal{F}$, ou seja,

$$
R\left(\hat{f}_{w s}, f\right) \approx i n f_{\hat{f}} s u p_{f \in \mathcal{F}} R(\hat{f}, f),
$$

onde $R$ é o risco do estimador $\hat{f}$ de $f$. Esse resultado significa que encolhimento de ondaletas fornece o melhor estimador possível de $f(t)$ fazendo uma suposição mínima sobre a natureza de $\mathcal{F}$. 
O procedimento de encolhimento de ondaletas pode ser dividido em duas etapas: escolhe-se o tipo de suavização (uma função $\delta_{c}(x)$ ) e escolhe-se o valor do truncamento $\left(\lambda_{j}\right)$. Duas formas básicas para a função $\delta_{c}(x)$ são "soft shrinkage" e "hard shrinkage" definidas por

$$
\delta_{c}^{s}(x)=\left\{\begin{aligned}
0, & \text { se }|x| \leq \lambda \\
\operatorname{sign}(x)(|x|-\lambda), & \text { se }|x|>c
\end{aligned}\right.
$$

e

$$
\delta_{c}^{h}(x)= \begin{cases}0, & \text { se }|x| \leq \lambda \\ x, & \text { se }|x|>\lambda\end{cases}
$$

respectivamente.

Existe uma discontinuidade na função "hard shrinkage" e a "soft shrinkage" é uma função contínua. A motivação para uso de "soft shrinkage" vem do princípio que o ruído afeta todos os coeficientes de ondaletas, além disso, a continuidade da função a torna preferível por razões estatísticas. Em algumas aplicações sobre a redução de vícios nos problemas de estimação, "hard shrinkage" é preferível.

Existem várias regras para selecionar o valor do truncamento $\lambda_{j}$. Algumas dessas regras são motivadas por teorias estatísticas e oferecem uma série de formas para truncar os coeficientes de ondaletas. Na prática, a mais utilizada é a universal, que é definida por $\lambda_{j}=\sqrt{2 \log (n)}$, onde $n$ é o tamanho da amostra.

Para outros tipos de $\lambda_{j}$, ver Donoho e Johnstone (1994), Bruce e Gao (1995, 1996). 


\section{Capítulo 3}

\section{Processos Estacionários e Não-Estacionários}

\subsection{Introdução}

$\mathrm{Na}$ análise de séries temporais, os processos estacionários desempenham um papel fundamental. As técnicas estatísticas para estes processos (métodos baseados na densidade espectral ou nos modelos paramétricos, tais como modelos ARMA) são bem desenvolvidas e são utilizadas frequentemente nas aplicações. Em geral, a suposição básica para desenvolver uma teoria assintótica é a estacionariedade devido ao fato de que esta garante que as estruturas do processo no presente contêm as informações do futuro do mesmo.

Não há uma generalização natural da definição de processo estacionário para processo não-estacionário. Um dos maiores problemas encontrados quando trabalhamos com um processo não-estacionário é como estabelecer uma teoria assintótica adequada. Considerações assintóticas são necessárias na análise de séries temporais para simplificar a análise, uma vez que os resultados obtidos baseados numa amostra de tamanho finito são normalmente viciados. Se $X_{1}, \cdots, X_{T}$ são observações de um processo não-estacionário arbitrário, então quando $T \rightarrow \infty$, isto é, estendendo o processo para o futuro, não se pode obter, em geral, nenhuma informação do comportamento do processo no intervalo inicial. Portanto, precisamos estabelecer um conceito assintótico diferente. 
Neste capítulo, introduzimos os conceitos necessários sobre processos estacionários e não-estacionários. Serão também apresentados alguns exemplos desses processos.

\subsection{Processos Estacionários}

Definição 3.1: Um processo estocástico $X=\{X(t, \omega), t \in T, \omega \in \Omega\}$ é uma família de variáveis aleatórias definidas num espaço de probabilidade $(\omega, \Lambda, P)$ e indexadas pelos elementos de um conjunto de parâmetros $T$.

$\mathrm{Na}$ realidade, um processo estocástico é uma funçào de dois argumentos, $X(t, \omega), t \in T, \omega \in \Omega$. Para cada $t \in T$, a função $X(t, \cdot)$ é mensurável relativamente a $\Lambda$. Por outro lado, para cada $\omega \in \Omega$, fixado, obteremos uma função $X(\cdot, \omega)$ de $t: T \rightarrow \mathcal{R}$, que é chamada trajetória, realização, função amostral do processo ou série temporal.

Para facilitar a notação, representaremos $X(t, \omega)$ por $X(t)$.

Definição 3.2: Dado um processo estocástico $\left\{X^{r}(t), t \in T\right\}$, definimos suas distribuições finito-dimensionais por

$$
F\left(x_{1}, \cdots, x_{n} ; t_{1}, \cdots, t_{n}\right)=\operatorname{Prob}\left(X\left(t_{1}\right) \leq x_{1}, \cdots, X\left(t_{n}\right) \leq x_{n}\right),
$$

para todo $n \geq 1$ e $t_{1}, \cdots, t_{n} \in T$.

Definição 3.3: As funções média e covariância de um processo $X(t)$ são dadas por

$$
\begin{aligned}
& E X(t)=\int_{-\infty}^{\infty} x d F(x ; t)=\mu_{x}(t) \\
& \operatorname{Cov}\left[X\left(t_{1}\right), X\left(t_{2}\right)\right]=\iint\left[x_{1}-\mu_{x}\left(t_{1}\right)\right]\left[x_{2}-\mu_{x}\left(t_{2}\right)\right] d F\left[x_{1}, x_{2} ; t_{1}, t_{2}\right] \\
&=E\left[X\left(t_{1}\right) X\left(t_{2}\right)\right]-E\left[X\left(t_{1}\right)\right] E\left[X\left(t_{2}\right)\right] \\
&=\gamma_{x x}\left(t_{1}, t_{2}\right) .
\end{aligned}
$$

Em particular, se $t_{1}=t_{2}$, temos a variância do processo

$$
\operatorname{Var} X(t)=\int\left[x-\mu_{x}(t)\right]^{2} d F(x ; t)=E\left[X^{2}(t)\right]-E^{2}\left[X^{\prime}(t)\right]=\gamma_{x x}(t, t) .
$$


Definição 3.4: Um processo estocástico $\{X(t), t \in T\}$ é estritamente estacionário se todas as distribuições finito-dimensionais (3.1) permanecem as mesmas sob uma translação do tempo, ou seja,

$$
F\left(x_{1}, \cdots, x_{n} ; t_{1}+\tau, \cdots, t_{n}+\tau\right)=F\left(x_{1}, \cdots, x_{n} ; t_{1}, \cdots, t_{n}\right),
$$

para quaisquer $t_{1}, \cdots, t_{n}, \tau \in T$.

Exemplo 3.1: (Série de Ruído Puro) Seja $\epsilon(t), t=0, \pm 1 \cdots$ uma sequência de variáveis aleatórias independentes, identicamente distribuidas. Essa série forma uma série temporal estritamente estacionária.

Exemplo 3.2: Seja $\epsilon(t)$ uma série de ruído puro. Então a série $X(t), t=$ $0, \pm 1, \cdots$ definida como

$$
X(t)=f[\epsilon(t), \epsilon(t-1), \epsilon(t+1), \cdots],
$$

é uma série temporal estritamente estacionária, onde $f(\cdot)$ é uma função deterministica.

Definição 3.5: Um processo estocástico $\{X(t), t \in T\}$ é estacionário de segunda ordem, ou fracamente estacionário, ou ainda estacionário em sentido amplo se

(i) $E[X(t)]=\mu_{x}$, constante, $\forall t \in T$;

(ii) $E\left[X^{2}(t)\right]<\infty, \forall t \in T$;

(iii) $\gamma_{x x}\left(t_{1}, t_{2}\right)=\gamma_{x x}\left(t_{1}-t_{2}\right)$ só depende da defasagem $\left|t_{1}-t_{2}\right|, \forall t_{1}, t_{2} \in T$.

Segue-se que $\operatorname{Cov}\{X(t), X(t+u)\}$ depende somente de $u$ e escrevemos

$$
\operatorname{Cov}\{X(t), X(t+u)\}=\gamma_{x x}(u), \quad u=0, \pm 1, \pm 2, \cdots
$$

e $\gamma_{x x}(u)$ é chamada a função de autocovariância de $\{X(t)\}$.

Definição 3.6: Um processo estocástico $\{X(t), t \in T\}$ é Gaussiano se, para quaisquer $t_{1}, \cdots, t_{n}$, o conjunto de variáveis aleatórias $\left\{X\left(t_{1}\right), \cdots, X\left(t_{n}\right)\right\}$ tem uma distribuição normal multivariada.

Exemplo 3.3: (Cossenoide) Suponha que $X(t)$ seja uma série de dimensão $r$ com componentes

$$
X_{j}(t)=R_{j} \cos \left(\omega_{j} t+\phi_{j}\right)
$$


para $j=1, \cdots, r$, onde $R_{1}, \cdots, R_{r}$ são constantes e $\phi_{1}, \cdots, \phi_{r-1}$ são uniforme em $(-\pi, \pi)$, e $\phi_{1}+\cdots+\phi_{r}=0$. Esta série é estacionária.

Exemplo 3.4: (Série Gaussiana Estacionária) Uma série de dimensão $r$ $X(t), t=0, \pm 1, \cdots$ é uma série Gaussiana se todas suas distribuições finitodimensionais são Gaussianas multivariadas (normais). Se $E X(t)=\mu \mathrm{e}$ $E X(t) X^{\prime}(u)=R(t-u)$ para todo $t, u$, então $X(t)$ é estacionária. Notamos que se $X(t)$ é uma série Gaussiana de dimensão $r$, então

$$
Y(t)=\sum_{u} a(t-u) X(u),
$$

para um filtro somável $\{a(t)\} s \times r$, é uma série Gaussiana estacionária de dimensão $s$.

Definição 3.7: (a) Seja $\left\{X_{1}, \cdots, X_{r}\right\}$ uma variável aleatória r-dimensional com $E\left|X_{j}\right|^{r}<\infty, j=1, \cdots, r$, onde $X_{j}$ são reais ou complexas. $O$ cumulante conjunto de ordem $r$ é dado por

$$
\operatorname{Cum}\left(X_{1}, \cdots, X_{r}\right)=\sum_{\nu}(-1)^{p-1}(p-1) !\left(E \prod_{j \in \nu_{1}} X_{j}\right) \cdots\left(E \prod_{j \in \nu_{p}} X_{j}\right),
$$

onde a soma é sobre todas as partições $\nu=\left(\nu_{1}, \cdots \nu_{p}\right)$ dos inteiros $(1, \cdots, r)$.

(b) Seja uma série temporal $\{X(t), t=0, \pm 1, \cdots\}$ satisfazendo $E|X(t)|^{k}<\infty$, então

$$
C_{x}\left(t_{1}, \cdots, t_{r}\right)=\operatorname{Cum}\left(X_{t_{1}}, \cdots, X_{t_{r}}\right)
$$

é denominada função cumulante conjunta de ordem $\mathrm{r}$ da série $X(t)$.

Resultado: $\mathrm{O} C u m\left(X_{1}, \cdots, X_{r}\right)$ é dado pelo coeficiente de

$$
i^{r} t_{1} \cdots t_{r}
$$

na série de Taylor de $\log \left(\operatorname{Exp}\left(i \sum_{j=1}^{r} X_{j} t_{j}\right)\right)$.

Definição 3.8: Suponha a série temporal $\{X(t), t=0, \pm 1, \cdots\}$, com função de autocovariância $\gamma_{x x}(u)$ satisfazendo

$$
\sum_{u=-\infty}^{\infty}\left|\gamma_{x x}(u)\right|<\infty
$$


então a função densidade espectral de $X(t)$ (ou simplesmente o espectro de $X(t))$, na frequência $\omega$, é dada por

$$
f(\omega)=\frac{1}{2 \pi} \sum_{u=-\infty}^{\infty} \gamma_{x x}(u) e^{-i \omega u}, \quad-\pi \leq \omega \leq \pi .
$$

A relação (3.9) pode ser invertida e a função de autocovariância $\gamma_{x x}(u)$ pode ser escrita como

$$
\gamma_{x x}(u)=\int_{-\pi}^{\pi} e^{i \omega u} f(\omega) d \omega, \quad u=0, \pm 1, \cdots .
$$

No caso particular $u=0$, temos

$$
\operatorname{Var}\{X(t)\}=\int_{-\pi}^{\pi} f(\omega) d \omega .
$$

No caso que a condição (3.8) não esteja satisfeita, podemos ainda representar $\gamma_{x x}(u)$ numa forma similar a (3.10), usando a integral de RiemannStieltjes,

$$
\gamma_{x x}(u)=\int_{-\pi}^{\pi} e^{i \omega u} d F(\omega),
$$

onde $F(\omega)$ é uma função não decrescente $\operatorname{com} F(-\pi)=0, F(\pi)=\sigma^{2}$, denominada o espectro integrado ou função distribuição espectral. Invertendo (3.12), temos

$$
F(\omega)=\sigma^{2}\left(\frac{\omega+\pi}{2 \pi}\right)+\frac{1}{2 \pi}\left[\sum_{u=-\infty}^{-1}+\sum_{u=1}^{\infty}\right] \frac{e^{-i u \omega}}{-u i} \gamma_{x x}(u) .
$$

Quando $f(\omega)$ existe, temos $d F(\omega)=f(\omega) d \omega$, tal que

$$
F(\omega)=\int_{-\pi}^{\omega} f(\theta) d \theta .
$$

Analogamente a (3.12), existe uma representação para $X(t)$, quando $E[X(t)]=$ 0 , isto é,

$$
X(t)=\int_{-\pi}^{\pi} e^{i \omega t} d Z(\omega)=\int_{-\pi}^{\pi} A(\omega) e^{i \omega t} d \zeta(\omega),
$$

para $t=0, \pm 1, \cdots$, onde $d Z(\omega)$ e $d \zeta(\omega)$ são processos estocásticos com incrementos ortogonais e ortonormais, respectivamente. Além disso, temos

$$
E\left[|d Z(\omega)|^{2}\right]=d F(\omega) .
$$


Se $F(\omega)$ for absolutamente contínua (com respeito à medida de Lebesgue),

$$
d F(\omega)=f(\omega) d \omega
$$

A representação (3.14) é conhecida como a representação espectral de $X(t)$. Na realidade, (3.14) nos diz que qualquer série estacionária com parâmetro discreto pode ser representada como uma soma de senos e cossenos involvendo um intervalo contínuo de frequências sobre $(-\pi, \pi)$, com amplitudes aleatórias, $|d Z(\omega)|$, e fases aleatórias, $\arg \{d Z(\omega)\}$. Podemos notar por (3.15), que $d F(\omega)$ é a amplitude quadrática média da componente em $X(t)$ com frequência $\omega$.

Para um processo estocástico contínuo $\{X(t),-\infty<t<\infty\}$ com média zero, sua representação espectral é

$$
X(t)=\int_{-\infty}^{\infty} e^{i t \omega} d Z(\omega)
$$

onde $\left\{d Z(\omega\}\right.$ um processo ortogonal sobre $(-\infty, \infty)$, com $E\left[|d Z(\omega)|^{2}\right]=$ $d F(\omega)$.

\subsection{Processos Não-Estacionários}

Seja $\{X(t),-\infty<t<\infty\}$ um processo estocástico com média zero e variância finita, isto é,

$$
E[X(t)]=0, \quad E\left[|X(t)|^{2}\right]<\infty,
$$

para todo $t$. Então a covariância é definida por

$$
\gamma(s, t)=E[\overline{X(s)} X(t)] .
$$

Se $\{X(t)\}$ for estacionário, isto é, se $\gamma(s, t)$ somente for uma função de $|s-t|$, então $\gamma(s, t)$ admite uma representação similar a (3.12),

$$
\gamma(s, t)=\int_{-\infty}^{\infty} e^{i \omega(t-s)} d F(\omega)
$$

onde $F(\omega)$, o espectro integrado, tem as propriedades de uma função distribuição no intervalo $(-\infty, \infty)$, e $X(t)$ admite a representação espectral (3.16). 
Se $X(t)$ for não-estacionário, ele não pode ser representado na forma (3.16) e, da mesma maneira $\gamma(s, t)$ também não pode ser representada na forma (3.18). Mas podemos representar $\gamma(s, t)$ numa forma similar a (3.18), substituindo a função $\left\{e^{i \omega t}\right\}$ por uma família de funções mais gerais, $\left\{\phi_{t}(\omega)\right\}$.

Agora consideremos a classe de processos para a qual existe uma família $\mathcal{F}$ de funções $\left\{\phi_{t}(\omega)\right\}$ definidas na reta real e indexadas por $t$, e uma medida $\mu(\omega)$ na reta real, tal que para cada $s, t, \gamma(s, t)$ admite uma representação da forma

$$
\gamma(s, t)=\int_{-\infty}^{\infty} \overline{\phi_{s}(\omega)} \phi_{t}(\omega) d \mu(\omega)
$$

e o processo $X(t)$ admite a representação

$$
X(t)=\int_{-\infty}^{\infty} \phi_{t}(\omega) d Z(\omega)
$$

onde $Z(\omega)$ é um processo ortogonal com

$$
E\left[|d Z(\omega)|^{2}\right]=d \mu(\omega) .
$$

Aqui, a medida $\mu(\omega)$ assume a mesma função que o espectro integrado $F(\omega)$ no caso estacionário.

Parzen (1959) ressaltou que se existe uma representação de $\{X(t)\}$ da forma (3.20), então existe um grande número de representações diferentes do processo, cada representação é baseada numa família diferente de funções. Quando o processo é estacionário, uma representação possível é

$$
\phi_{t}(\omega)=e^{i \omega t}
$$

Se desejarmos introduzir a noção de frequência na análise de processos não-estacionários, devemos procurar novas famílias de funções que embora sejam não-estacionárias, têm uma forma oscilatória na qual a noção de frequência ainda é dominante. Uma família de funções que possui a estrutura requerida pode ser obtida como segue. Ver Priestley $(1965,1981)$.

Suponha que, para cada $\omega$ fixada, o valor máximo absoluto das transformadas de Fourier de $\phi_{t}(\omega)$ dá-se na frequência $\theta(\omega)$. Então podemos considerar $\phi_{t}(\omega)$ como uma senóide com amplitude modulada, com frequência $\theta(\omega)$, e $\phi_{t}(\omega)$ pode ser escrita na forma

$$
\phi_{t}(\omega)=A_{t}(\omega) e^{i \theta(\omega) t},
$$


onde a função $A_{t}(\omega)$ é tal que o valor máximo absoluto de suas transformadas de Fourier é na origem. isto é, $\theta(\omega)=0$.

A função $\phi_{t}(\omega)$ em $t$ será chamada uma função oscilatória se, para alguma $\theta(\omega)$, ela pode ser escrita na forma (3.22) onde $A_{t}(\omega)$ é da forma

$$
A_{t}(\omega)=\int_{-\infty}^{\infty} e^{i t \theta} d K_{\omega}(\theta)
$$

com máximo de $\left|d K_{\omega}(\theta)\right|$ em $\theta=0$.

Se a função $\left\{\phi_{t}(\omega)\right\}$ é tal que $\theta(\omega)$ é uma função de $\omega$, então (3.19) pode ser escrita na forma

$$
\gamma(s, t)=\int_{-\infty}^{\infty} \overline{A_{s}(\omega)} A_{t}(\omega) e^{i \omega(t-s)} d \mu(\omega)
$$

$\mathrm{e}$

$$
X(t)=\int_{-\infty}^{\infty} A_{t}(\omega) e^{i \omega t} d Z(\omega),
$$

onde $E\left[|d Z(\omega)|^{2}\right]=d \mu(\omega)$.

Se existe uma família de funções oscilatórias $\left\{\phi_{t}(\omega)\right\}$ tal que o processo $\{X(t)\}$ tem uma representação da forma (3.20), então $X(t)$ é chamado um processo oscilatório.

Se qualquer processo oscilatório tiver uma representação da forma (3.25), onde a família $A_{t}(\omega)$ satisfaz (3.23), então podemos escrever essas famílias de funções oscilatórias na forma

$$
\phi_{t}(\omega)=A_{t}(\omega) e^{i \omega t}
$$

Como (3.21) é um caso particular de (3.22) (com $A_{t}(\omega)=1$, para todo $t, \omega$ e $\theta(\omega)=\omega)$, a classe de processos oscilatórios certamente inclui todos os processos estacionários de segunda ordem.

A partir de (3.24) temos

$$
\operatorname{Var}\{X(t)\}=\gamma(t, t)=\int_{-\infty}^{\infty}\left|A_{t}(\omega)\right|^{2} d \mu(\omega) .
$$

Como $\operatorname{Var}\{X(t)\}$ pode ser interpretada como uma medida da potência total do processo no tempo $t$, (3.26) fornece uma decomposição da potência total no qual a contribuição da frequência $\omega$ é $\left\{\left|A_{t}(\omega)\right|^{2} d \mu(\omega)\right\}$. 
Seja $\mathcal{F}$ uma família particular de funções oscilatórias $\left\{\phi_{t}(\omega)\right\}=\left\{A_{t}(\omega) e^{i \omega t}\right\}$ e $\{X(t)\}$ um processo oscilatório tendo uma representação da forma (3.25) em termos da família $\mathcal{F}$. Priestley (1981) definiu o espectro evolucionário no tempo $t$ com respeito à família $\mathcal{F}, d F_{t}(\omega)$, por

$$
d F_{t}(\omega)=\left|A_{t}(\omega)\right|^{2} d \mu(\omega) \text {. }
$$

Note que quando $\{X(t)\}$ é estacionário e $\mathcal{F}$ é escolhida para ser a família $\left\{e^{i \omega t}\right\}, d F_{t}(\omega)$ reduz-se à definição padrão do espectro. O espectro evolucionário tem a mesma interpretação física que o espectro de um processo estacionário, isto é, ele descreve uma distribuição da potência sobre frequências. Mas no processo estacionário, o espectro é determinado pelo comportamento do processo sobre todo tempo e no processo não-estacionário, (3.27) representa especificamente o espectro do processo na vizinhança do instante $t$.

Embora $d F_{t}(\omega)$ dependa de $\mathcal{F}$, a partir de (3.26), temos

$$
\operatorname{Var}\{X(t)\}=\int_{-\infty}^{\infty} d F_{t}(\omega)
$$

isto é, $\operatorname{Var}\{X(t)\}$ independe de $\mathcal{F}$, e para todas as famílias, ela representa a potência total do processo no tempo $t$.

É conveniente padronizar a função $A_{t}(\omega)$, para que $A_{0}(\omega)=1$, para todo $\omega$, isto é, incorporamos $\left|A_{0}(\omega)\right|$ na medida $\mu(\omega)$. Com essa convenção, $d \mu(\omega)$ representa o espectro evolucionário no tempo $t=0$, e $\left|A_{t}(\omega)\right|^{2}$ representa a mudança no espectro em relação a $t=0$. Temos, para cada $\omega$,

$$
\int_{-\infty}^{\infty} d K_{\omega}(\theta)=1
$$

para que as transformadas de Fourier de $\left\{A_{t}(\omega)\right\}$ sejam normalizadas.

Quando $\mu(\omega)$ é absolutamente contínua com respeito à medida de Lebesgue, podemos escrever, para cada $t$,

$$
d F_{t}(\omega)=f_{t}(\omega) d \omega
$$

e $f_{t}(\omega)$ é a função densidade espectral evolucionária.

Para um processo oscilatório com parâmetro discreto, $X(t)$ tem uma representação da forma (3.25) com os limites de integral em $(-\pi, \pi)$. 
Além do espectro evolucionário de Priestley, varias outras definições do espectro dependendo do tempo para processos não-estacionários foram propostas na literatura. Algumas delas são:

\section{O espectro instantâneo de Page.}

Page (1952) introduziu a noção de espectro instantâneo. Dado um processo $\{X(t),-\infty<t<\infty\}$, Page introduziu a quantidade

$$
g_{T}(\omega)=\left|\int_{0}^{T} X(t) e^{-i \omega t} d t\right|^{2}
$$

isto é, proporcional ao periodograma das observações no intervalo $(0, T)$ e definiu o espectro $f(\omega)$ do processo como

$$
f(\omega)=\lim _{T \rightarrow \infty} E\left\{g_{T}(\omega)\right\} .
$$

Então o espectro instantâneo, $\rho_{t}(\omega)$, satisfaz a relação

$$
E\left\{g_{T}(\omega)\right\}=\int_{0}^{T} \rho_{t}(\omega) d t
$$

para que

$$
\rho_{t}(\omega)=\frac{d}{d t} E\left\{g_{t}(\omega)\right\}
$$

$\mathrm{e}$

$$
f(\omega)=\int_{0}^{\infty} \rho_{t}(\omega) d t
$$

\section{O espectro de Wigner-Ville}

Seja $\{X(t),-\infty<t<\infty\}$ um processo não-estacionário, com covariância $\gamma(s, t)$. O espectro de Wigner-Ville, $\Psi_{t}(\omega)$, é definido como a transformada de Fourier de $\gamma(t-\tau / 2, t+\tau / 2) \operatorname{com} t$ fixo. Então, $\Psi_{t}(\omega)$ é dado por

$$
\Psi_{t}(\omega)=\frac{1}{2 \pi} \int_{-\infty}^{\infty} \gamma(t-\tau / 2, t+\tau / 2) e^{-i \omega \tau} d \tau .
$$

\section{Aproximação de Tjøstheim.}

Tjøstheim (1976) propõe uma definição do espectro dependendo do tempo baseado na representação de um processo em termos de suas inovações. 
Dado um processo $\{X(t), t=0, \pm 1, \cdots\}$, ele tem a representação linear da forma

$$
X(t)=\sum_{u=0}^{\infty} a_{t}(u) \epsilon_{t-u},
$$

onde $\left\{\epsilon_{t}\right\}$ é uma sequência de ruído branco, com variância $\sigma_{\epsilon}^{2}$. Então Tjøstheim definiu o espectro no tempo $t$ por

$$
P_{t}(\omega)=\frac{\sigma_{\epsilon}^{2}}{2 \pi}\left|\sum_{u=0}^{\infty} a_{t}(u) e^{-i \omega u}\right|^{2} .
$$

Ver Flandrin(1989) para detalhes.

Agora, consideremos um processo oscilatório bivariado com parâmetro contínuo $\{X(t), Y(t)\}$, onde

$$
X(t)=\int_{-\infty}^{\infty} A_{t, X}(\omega) e^{i \omega t} d Z_{X}(\omega)
$$

$\mathrm{e}$

$$
Y(t)=\int_{-\infty}^{\infty} A_{t, Y}(\omega) e^{i \omega t} d Z_{Y}(\omega)
$$

com

$$
\begin{gathered}
E\left[d Z_{X}(\omega) \overline{d Z_{X}\left(\omega^{\prime}\right)}\right]=E\left[d Z_{Y}(\omega) \overline{d Z_{Y}\left(\omega^{\prime}\right)}\right]=E\left[d Z_{X}(\omega) \overline{d Z_{Y^{\prime}}\left(\omega^{\prime}\right)}\right]=0, \quad \omega \neq \omega^{\prime}, \\
E\left[\left|d Z_{X}(\omega)\right|^{2}\right]=d \mu_{X X}(\omega), \\
E\left[\left|d Z_{Y}(\omega)\right|^{2}\right]=d \mu_{Y Y}(\omega), \\
E\left[d Z_{X}(\omega) \overline{d Z_{Y}(\omega)}\right]=d \mu_{X Y}(\omega) .
\end{gathered}
$$

Priestley (1965) definiu o espectro evolucionário cruzado no tempo $t$ com respeito às famílias $\mathcal{F}_{\mathcal{X}}$ e $\mathcal{F}_{\mathcal{Y}}, d F_{t, X Y}(\omega)$, por

$$
d F_{t, X Y}(\omega)=A_{t, X}(\omega) \overline{A_{t, Y}(\omega)} d \mu_{X Y}(\omega),
$$

onde $\mathcal{F}_{\mathcal{X}}$ e $\mathcal{F}_{y}$ são famílias de funções oscilatórias $\left\{\phi_{t, X}(\omega)=A_{t, X}(\omega) e^{i \omega t}\right\}$, $\left\{\phi_{t, Y}(\omega)=A_{t, Y}(\omega) e^{i \omega t}\right\}$, respectivamente. 


\subsection{Processos Localmente Estacionários}

Na seção anterior, Priestley (1981) considerou processos que têm uma representação espectral da forma:

$$
X_{t}=\int_{-\pi}^{\pi} \exp (i \omega t) A_{t}(\omega) d Z(\omega) .
$$

Dentro do enfoque dele, não é possivel estabelecer a inferência assintótica desses processos, isto é, ferramentas assintóticas (consistência, normalidade assintótica, eficiência, etc) não podem ser aplicadas no tratamento teórico dos procedimentos estatísticos para tais processos.

Para superar esse problema. Dahlhaus (1997) introduziu uma classe de processos não-estacionários chamados localmente estacionários.

Definição 3.9: Uma sequência de processos estocásticos $\left\{X_{t, T}, t=1, \cdots, T\right\}$ é chamada localmente estacionária com função de transferência $A^{\circ}$ e tendência $\mu$ se existe uma representação

$$
X_{t, T}=\mu\left(\frac{t}{T}\right)+\int_{-\pi}^{+\pi} \exp (i \omega t) A_{t, T}^{o}(\omega) d \xi(\omega)
$$

tal que:

(i) $\xi(\omega)$ é um processo estocástico em $[-\pi, \pi] \operatorname{com} \overline{\xi(\omega)}=\xi(-\omega)$ e

$$
\operatorname{cum}\left\{d \xi\left(\omega_{1}\right), \cdots, d \xi\left(\omega_{k}\right)\right\}=\eta\left(\sum_{j=1}^{k} \omega_{j}\right) g_{k}\left(\omega_{1}, \cdots, \omega_{k-1}\right) d \omega_{1} \cdots d \omega_{k}
$$

onde $\operatorname{cum}\{\cdots\}$ denota o cumulante de k-ésima ordem, $g_{1}(\omega)=0$, $g_{2}(\omega)=1,\left|g_{k}\left(\omega_{1}, \cdots, \omega_{k-1}\right)\right| \leq$ const $_{k}$ para todo $k$ e $\eta(\omega)=\sum_{j=-\infty}^{\infty} \delta(\omega+$ $2 \pi j$ ) é uma extensão da função delta de Dirac com período $2 \pi$.

(ii) Existe um constante $K$, e uma função periódica com período $2 \pi, A$ : [0, 1$]$ $\mathrm{x} \mathcal{R} \rightarrow \mathcal{C} \operatorname{com} A(u,-\omega)=\overline{A(u, \omega)} \mathrm{e}$

$$
\sup _{t, \omega}\left|A_{t, T}^{o}(\omega)-A\left(\frac{t}{T}, \omega\right)\right| \leq K T^{-1},
$$

para todo $T$. Supomos que $A(u, \omega)$ e $\mu(u)$ sejam contínuas em $u$. 
A suavização ("smoothness") de $A$ em u garante que o processo tem um comportamento localmente estacionário. A quantidade

$$
f(u, \omega)=|A(u, \omega)|^{2}
$$

é chamada espectro dependente do tempo do processo e Dahlhaus (1996) provou que $f(u, \omega)$ é univocamente determinada.

Exemplo 3.5: Suponha $Y_{t}$ seja um processo estacionário e $\mu, \sigma:[0,1] \rightarrow R$ são contínuos. Então

$$
X_{t, T}=\mu\left(\frac{t}{T}\right)+\sigma\left(\frac{t}{T}\right) Y_{t}
$$

é localmente estacionário com $A_{t, T}^{o}(\omega)=A\left(\frac{t}{T}, \omega\right)$.

Exemplo 3.6: Suponha $X_{t, T}$ um processo de ARMA com coeficientes variando no tempo,

$$
\sum_{j=0}^{p} a_{j}\left(\frac{t}{T}\right) X_{t-j, T}=\sum_{j=0}^{q} b_{j}\left(\frac{t}{T}\right) \sigma\left(\frac{t-j}{T}\right) \epsilon_{t-j},
$$

onde $\epsilon_{t}$ são independentes, identicamente distribuidos com média zero e variância 1 e $a_{0}(u)=b_{0}(u)=1$. Então $X_{t, T}$ é localmente estacionário com espectro dependente do tempo

$$
f(u, \omega)=\frac{\sigma^{2}(u)\left|\sum_{j=0}^{q} b_{j}(u) \exp (i \omega j)\right|^{2}}{2 \pi\left|\sum_{j=0}^{p} a_{j}(u) \exp (i \omega j)\right|^{2}} .
$$

Dahlhaus (1996) definiu a covariância local de lag $k$ e tempo $u$ como

$$
C(u, k)=\int_{-\pi}^{\pi} f(u, \omega) \exp (i \omega k) d \omega
$$

e provou que

$$
\begin{aligned}
\operatorname{Cov}\left(X_{[u T], T}, X_{[u T]+k, T}\right) & =\int_{-\pi}^{\pi} \exp (i \omega k) A_{[u T]+k, T}^{o}(\omega) A_{[u T], T}^{o}(-\omega) d \omega \\
& =C(u, k)+O\left(T^{-1}\right)
\end{aligned}
$$

uniformemente em $u$ e $k$, onde $u=\frac{t}{T}$.

Dados os valores $X_{1, T}, \cdots, X_{T, T}$, o periodograma segmentado é definido sobre um segmento de tamanho $N$ com ponto médio $[u T]$,

$$
I_{N}(u, \omega)=\frac{1}{2 \pi H_{N}}\left|d_{N}(u, \omega)\right|^{2}
$$

onde 
(i) $d_{N}(u, \omega)=\sum_{s=0}^{N-1} h\left(\frac{s}{N}\right) X_{[u T]-\frac{N}{2}+s+1, T} \exp (-i \omega s)$,

(ii) $h:[0,1] \rightarrow \mathcal{R}$ é uma janela de dados ("taper"),

(iii) $H_{N}=\sum_{s=0}^{N-1}\left\{h\left(\frac{s}{N}\right)\right\}^{2}$.

Cada segmento é composto por $N$ pontos. O primeiro segmento será o intervalo $[0, N]$ com ponto médio $t_{1}=\frac{N}{2}$. O j-ésimo segmento será o intervalo $[(j-1) S ; N+(j-1) S]$ com ponto médio $t_{j}=(j-1) S+\frac{N}{2}$, para $j=1, \cdots, M$, onde $T=S(M-1)+N$.

O periodograma suavizado é definido como

$$
\hat{f}(u, \omega)=\left(b_{f}\right)^{-1} \int K_{f}\left(\frac{\omega-\mu}{b_{f}}\right) I_{N}(u, \mu) d \mu
$$

onde

(i) $K_{f}: R \rightarrow[0, \infty)$ é um núcleo com $K_{f}(x)=0$, para $x \notin[-1 / 2,1 / 2]$;

(ii) $K_{f}(x)=K_{f}(-x)$;

(iii) $\int K_{f}(x) d x=1$;

(iv) $b_{f}$ é a largura da faixa na direção de frequência.

A covariância local $C(u, k)$ pode ser estimada por

$$
\hat{C}_{T}(u, k)=\left(b_{T} T\right)^{-1} \sum_{t} K\left(\frac{u-(t+k / 2) / T}{b_{T}}\right) X_{t, T} X_{t+k, T}
$$

onde

(a) $K: R \rightarrow[0, \infty)$ é um núcleo com $K^{\prime}(x)=K^{\prime}(-x)$,

(b) $\int K(x) d x=1$,

(c) $K(x)=0$ para $x \notin[-1 / 2,1 / 2]$,

(d) $b_{T}$ é a largura da faixa no tempo.

Dahlhaus (1996) provou alguns resultados assintóticos sobre estes estimadores, a saber: 
(1) $\hat{C}_{T}(u, k)$ é um estimador assintoticamente não viciado de $C(u, k)$;

(2) o periodograma $I_{N}(u, \lambda)$ é um estimador assintoticamente não viciado de $f(u, \omega)$, mas não consistente.

(3) o periodograma suavizado $\hat{f}(u, \omega)$ também é um estimador assintoticamente não viciado de $f(u, \omega)$. Além disso, ele é um estimador consistente. 


\section{Capítulo 4}

\section{Análise Espectral via Ondaletas}

\subsection{Introdução}

Uma das maneiras de descrever séries temporais é através de modelos não-paramétricos, ou seja, modelos com um número infinito de parâmetros. Um dos modelos não-paramétricos mais utilizados é a análise espectral via exponenciais (análise de Fourier), no domínio de frequência, ou a análise da função de autocovariância, no domínio do tempo.

Em geral, podemos dizer que a análise de Fourier de uma série temporal consiste em decompor esta série em uma soma de funções senos e cossenos, a partir de registros de comprimento finito.

$\mathrm{Na}$ análise de Fourier, a característica funtamental é o espectro, que é a transformada de Fourier da função de autocovariância. Logo, sob o ponto de vista da quantidade de informação probabilística que fornecem, o espectro e a função de autocovariância são ferramentas equivalentes. A análise de Fourier é fundamental em áreas onde o interesse básico é a procura de periodicidades nos dados e tem aplicações nos campos de engenharia elétrica, comunicações, fisica, economia, medicina, meteorologia, oceanografia, etc.

Recentemente, surgiu uma nova técnica chamada de ondaletas, com aplicações em processamento e codificação de sinais e imagens, compressão de dados e imagens, análise numérica, estudo de turbulência, etc. Neste capítulo, desenvolvemos uma análise espectral via ondaletas para um processo estacionário discreto. Diferente da análise de Fourier, esta análise é feita em 
tempo-escala. Ou seja, na análise de Fourier temos um parâmetro, a frequência, e na análise de ondaletas temos dois parâmetros, tempo e escala. $\mathrm{Na}$ seção 4.2 apresentamos o conceito de espectro de ondaletas e alguns exemplos. Na seção 4.3 provamos algumas propriedades assintóticas da transformada finita de ondaletas. O periodograma de ondaletas é discutido na seção 4.4. A seção 4.5 apresenta o método do escalograma e uma série temporal harmônica é construida para ilustrar esse método e na seção 4.6 o procedimento de encolhimento de ondaletas é desenvolvido. Finalmente, na seção 4.7 apresentamos uma aplicação.

\subsection{O Espectro de Ondaletas}

Nesta seção definimos o espectro de ondaletas de uma série temporal estacionária $\{X(t), t \in Z\}$, com respeito a uma ondaleta $\psi$.

Suposição 4.1: Assumimos que $\{X(t), t=0, \pm 1, \cdots\}$ seja uma série temporal discreta, com média zero, estacionária de segunda ordem. Seja $\gamma(u)=$ $E\{X(t) X(t+u)\}, u=0, \pm 1, \cdots$, a função de autocovariância de $X(t)$.

Em nossas considerações, tomaremos $X(0), X(1), \cdots, X(T-1)$ com $T=2^{M}$, como uma amostra da série $\{X(t), t \in Z\}$, onde $M$ é um inteiro positivo.

\section{Suposição 4.2:}

$$
\sum_{u=-\infty}^{\infty}[1+|u|]|\gamma(u)|<\infty .
$$

Suponha que as suposições 4.1 e 4.2 estão satisfeitas, então o espectro de ondaletas da série $\{X(t), t=0, \pm 1, \cdots\}$, em $(j, k)$, com respeito a $\psi$, é definido como

$$
\eta_{j, k}^{(\psi)}=\sum_{u=-\infty}^{\infty} \gamma(u) \Psi_{j, k}(u), \quad j, k \in Z
$$

onde

$$
\Psi_{j, k}(u)=\sum_{t=0}^{\infty} \psi_{j, k}(t) \psi_{j, k}(t+|u|)
$$


é chamada a função de autocorrelação de ondaletas, em $(j, k)$, e $\psi_{j, k}(\cdot)$ é definida em (2.6).

Observação: $O$ espectro de ondaletas $\eta_{j, k}^{(\psi)}$ mede a potência local na decomposição de variância-covariância do processo $\{X(t)\}$ numa certa escala $j$ e uma localização de tempo $2^{j} k$. Para $j \geq 0$ fixo, essa quantidade é constante sobre a localização de tempo $2^{j} k$, isto é, a distribuição de $\eta_{j, k}^{(\psi)}$ é independente de $k$.

Teorema 4.1: Sob as suposições 4.1 e 4.2, $\eta_{j, k}^{(\psi)}$ é limitado e não negativo.

\section{Demonstração:}

Como $\psi$ é limitada e tem suporte compacto, então existe $A \in \Re$, um limite finito, tal que $|\psi| \leq A$ e $\mid$ support $\psi \mid<\infty$. Mas sabemos que $\psi_{j, k}(t)=$ $2^{-j / 2} \psi\left(2^{-j} t-k\right)$, portanto temos

$$
\left|\psi_{j, k}(\cdot)\right| \leq 2^{-j / 2} A
$$

e

$$
\mid \text { support } \psi_{j, k}\left|=2^{j}\right| \text { support } \psi \mid \text {. }
$$

Denotamos por $V$ a variação de $\psi$.

Agora, seja

$$
S_{T}^{(\psi)}(j, k)=\sum_{u=-T}^{T} \sum_{t=0}^{T} \gamma(u) \psi_{j, k}(t) \psi_{j, k}(t+|u|), \quad j, k \in Z
$$

Para $u>0$, (o caso de $u<0$ segue-se da mesma maneira),

$$
\begin{aligned}
& \left|\sum_{t} \psi_{j, k}(t+u) \psi_{j, k}(t)-\sum_{t} \psi_{j, k}(t) \psi_{j, k}(t)\right| \\
& \quad \leq \sum_{t}\left|\psi_{j, k}(t)\right|\left|\psi_{j, k}(t+u)-\psi_{j, k}(t)\right| \\
& \quad \leq 2^{-j / 2} A \sum_{t} \mid \psi_{j, k}(t+u)-\psi_{j, k}(t+u-1)+\psi_{j, k}(t+u-1)-\psi_{j, k}(t+u-2)+ \\
& \quad+\psi_{j, k}(t+u-2)-\cdots+\psi_{j, k}(t+1)-\psi_{j, k}(t) \mid
\end{aligned}
$$




$$
\begin{aligned}
& \leq 2^{-j / 2} A \sum_{t}\left\{\left|\psi_{j, k}(t+u)-\psi_{j, k}(t+u-1)\right|+\left|\psi_{j, k}(t+u-1)-\psi_{j, k}(t+u-2)\right|+\right. \\
& \left.+\cdots+\left|\psi_{j, k}(t+1)-\psi_{j, k}(t)\right|\right\} \\
& \leq 2^{-j / 2} A 2^{-j / 2} V|u| \\
& =2^{-j} A V|u| .
\end{aligned}
$$

Portanto,

$$
\begin{aligned}
S_{T}^{(\psi)}(j, k) & =\sum_{u=-T}^{T} \gamma(u) \sum_{t=0}^{T} \psi_{j, k}(t) \psi_{j, k}(t+|u|) \\
& =\sum_{u=-T}^{T} \gamma(u) \sum_{t=0}^{T} \psi_{j, k}(t) \psi_{j, k}(t)+\epsilon_{T} \\
& \leq \sum_{u=-T}^{T} \gamma(u) A^{2} \mid \text { support } \psi \mid+\epsilon_{T},
\end{aligned}
$$

pois,

$$
\begin{aligned}
\sum_{t=0}^{T} \psi_{j, k}(t) \psi_{j, k}(t) & \leq\left(2^{-j / 2} A\right)^{2} \mid \text { support } \psi_{j, k} \mid \\
& \leq 2^{-j} A^{2} 2^{j} \mid \text { support } \psi \mid \\
& \leq A^{2} \mid \text { support } \psi \mid
\end{aligned}
$$

Também,

$$
\begin{aligned}
\left|\epsilon_{T}\right| & \leq \sum_{u=-T}^{T}|\gamma(u)| 2^{-j} A V|u| \\
& \leq 2^{-j} A V \sum_{u=-T}^{T}|u \| \gamma(u)| .
\end{aligned}
$$

Daí, pela suposição 4.2 , temos que

$$
\eta_{j, k}^{(\psi)}=\lim _{T \rightarrow \infty} S_{T}^{(\psi)}(j, k)<\infty .
$$

Agora, seja $I_{j, k}^{(\psi)}=\left[\sum_{t=0}^{T-1} X(t) \psi_{j, k}(t)\right]^{2} \geq 0$. Então, 


$$
\begin{aligned}
E\left[I_{j, k}^{(\psi)}\right] & =\sum_{t=0}^{T-1} \sum_{s=0}^{T-1} E[X(t) X(s)] \psi_{j, k}(t) \psi_{j, k}(s) \\
& =\sum_{t=0}^{T-1} \sum_{s=0}^{T-1} \gamma(t-s) \psi_{j, k}(t) \psi_{j, k}(s) \\
& =\sum_{u=-(T-1)}^{T-1} \gamma(u) \sum_{t=0}^{T-|u|} \psi_{j, k}(t) \psi_{j, k}(t+|u|) .
\end{aligned}
$$

Portanto, como $T \rightarrow \infty, E\left[I_{j, k}^{(\psi)}\right] \rightarrow \eta_{j, k}^{(\psi)}$ pois a suposição 4.2 está satisfeita, daí $\eta_{j, k}^{(\psi)} \geq 0$.

Observação: $I_{j, k}^{(\psi)}=\left[\sum_{t=0}^{T-1} X(t) \psi_{j, k}(t)\right]^{2}$ é chamado o periodograma de ondaletas dos valores $X(0), \cdots, X(T-1)$ com respeito a $\psi$. Ele é um estimador assintoticamente não viciado do espectro $\eta_{j, k}^{(\psi)}$.

Agora apresentamos alguns exemplos de espectro de ondaletas com respeito à ondaleta de Haar.

Exemplo 4.1: Se $\{\varepsilon(t), t=0,1, \cdots\}$ é uma sequência de ruído branco, com média zero e variância $\sigma^{2}$, então,

$$
\begin{aligned}
\eta_{j, k}^{(H)} & =\sum_{u=-\infty}^{\infty} \gamma(u) \Psi_{j, k}^{(H)}(u) \\
& =\sum_{u=-\infty}^{\infty} \gamma(u) \sum_{t=0}^{\infty} \psi_{j, k}^{(H)}(t) \psi_{j, k}^{(H)}(t+|u|) \\
& =\sigma^{2} \sum_{t=0}^{\infty}\left[\psi_{j, k}^{(H)}(t)\right]^{2} \\
& =\left[2^{-j} I_{\left\{j<0, k \equiv 0\left(\bmod 2^{-\jmath}\right)\right\}}+I_{\{j \geq 0\}}\right] \sigma^{2} I_{\{k \geq 0\}} .
\end{aligned}
$$

Portanto, para $j \geq 0, k \geq 0, \eta_{j, k}^{(H)}=\sigma^{2}$, isto é, constante. Isso sugere que se acharmos um espectro constante para $j \geq 0, k \geq 0$, podemos estar na presença de uma série de ruído branco.

A figura B.1 mostra $\eta_{j, k}^{(H)}$ de $\varepsilon(t), \operatorname{com} \sigma=1$.

Exemplo 4.2: Vamos considerar um modelo de médias móveis de primeira 
ordem, MA(1),

$$
X(t)=\varepsilon(t)-\beta \varepsilon(t-1),
$$

$\operatorname{com} \varepsilon_{t} \sim R B\left(0, \sigma^{2}\right)$. Obtemos imediatamente

$$
\gamma(u)=\left\{\begin{array}{rc}
\left(1+\beta^{2}\right) \sigma^{2}, & u=0 \\
-\beta \sigma^{2}, & |u|=1 \\
0, & |u| \geq 2
\end{array}\right.
$$

então,

$$
\begin{aligned}
\eta_{j, k}^{(H)} & =\sum_{u=-\infty}^{\infty} \gamma(u) \Psi_{j, k}^{(H)}(u) \\
& =\sum_{u=-\infty}^{\infty} \gamma(u) \sum_{t=0}^{\infty} \psi_{j, k}^{(H)}(t) \psi_{j, k}^{(H)}(t+|u|) \\
& =\gamma(0) \sum_{t=0}^{\infty} \psi_{j, k}^{(H)}(t) \psi_{j, k}^{(H)}(t)+2 \gamma(1) \sum_{t=0}^{\infty} \psi_{j, k}^{(H)}(t) \psi_{j, k}^{(H)}(t+1) \\
& =\left\{\gamma(0)\left[2^{-j} I_{\left\{j<0, k \equiv 0\left(\bmod 2^{-\jmath}\right)\right\}}+I_{\{j \geq 0\}}\right]+2 \gamma(1)\left[1-\frac{3}{2^{j}}\right] I_{\{j \geq 1\}}\right\} I_{\{k \geq 0\}} .
\end{aligned}
$$

Em particular, com $\beta=0.7$, temos $\eta_{j, k}^{(H)}=0.09 \sigma^{2}$, quando $j \rightarrow \infty$ e $k \geq 0$.

A figura B.2 mostra $\gamma(u)$ e $\eta_{j, k}^{(H)}, \operatorname{com} \beta=0.7$ e $\sigma=1$.

Exemplo 4.3: Vamos considerar um modelo de auto-regressivo de primeira ordem, AR(1):

$$
X(t)=\alpha X(t-1)+\varepsilon(t), \quad|\alpha|<1,
$$

onde $\varepsilon_{t} \sim R B\left(0, \sigma^{2}\right)$. Portanto obtemos

$$
\gamma(u)= \begin{cases}\frac{1}{1-\alpha^{2}} \sigma^{2}, & u=0 \\ \alpha^{u} \gamma(0), & u \geq 1\end{cases}
$$

Então,

$$
\eta_{j, k}^{(H)}=\sum_{u=-\infty}^{\infty} \gamma(u) \Psi_{j, k}^{(H)}(u)
$$




$$
\begin{aligned}
= & \sum_{u=-\infty}^{\infty} \gamma(u) \sum_{t=0}^{\infty} \psi_{j, k}^{(H)}(t) \psi_{j, k}^{(H)}(t+|u|) \\
= & \gamma(0) \sum_{t=0}^{\infty} \psi_{j, k}^{(H)}(t) \psi_{j, k}^{(H)}(t)+2 \gamma(1) \sum_{t=0}^{\infty} \psi_{j, k}^{(H)}(t) \psi_{j, k}^{(H)}(t+1) \\
& +2 \gamma(2) \sum_{t=0}^{\infty} \psi_{j, k}^{(H)}(t) \psi_{j, k}^{(H)}(t+2)+\cdots \\
= & \gamma(0)\left\{\left[2^{-j} I_{\left\{j<0, k \equiv 0\left(\bmod 2^{-\jmath}\right)\right\}}+I_{\{j \geq 0\}}\right]+2 \alpha\left[1-\frac{3}{2^{j}}\right] I_{\{j=1\}}\right. \\
& \left.+\left\{2 \sum_{u=0}^{2^{(j-1)}} \alpha^{u}\left[1-\frac{3 u}{2^{j}}\right]-2 \sum_{u=2^{(j-1)}+1}^{2^{\jmath}-1} \alpha^{u}\left[1-\frac{u}{2^{j}}\right]\right\} I_{\{j>1\}}\right\} I_{\{k \geq 0\}} .
\end{aligned}
$$

Em particular, com $\alpha=0.8$, temos $\eta_{j, k}^{(H)}=25 \sigma^{2}$ quando $j \rightarrow \infty$ e $k \geq 0$.

A figura B.3 mostra $\gamma(u)$ e $\eta_{j, k}^{(H)}$, com $\alpha=0.8$ e $\sigma=1$.

\subsection{A Transformada Finita de Ondaletas e Suas Propriedades Assintóticas}

Nesta seção desenvolvemos algumas propriedades estocásticas da transformada finita de ondaletas da sequência $X(t), t=0,1, \cdots, T-1$. Aqui, $\{X(t), t \in Z\}$ é um processo estacionário discreto com média zero e $T=2^{M}$.

Para $j=1,2, \cdots, M$ e $k=0,1, \cdots,\left(2^{(M-j)}-1\right)$, definimos a transformada finita de ondaletas com respeito a $\psi$ como

$$
d_{j, k}^{(\psi)}=\sum_{t=0}^{T-1} X(t) \psi_{j, k}(t) .
$$

De (4.4) temos que,

$$
E\left\{d_{j, k}^{(\psi)}\right\}=0
$$

$\mathrm{e}$

$$
\begin{aligned}
\operatorname{Var}\left\{d_{j, k}^{(\psi)}\right\} & =\sum_{t=0}^{T-1} \sum_{s=0}^{T-1} \gamma(t-s) \psi_{j, k}(t) \psi_{j, k}(s) \\
& =\sum_{u=-(T-1)}^{T-1} \gamma(u) \sum_{t=0}^{T-1-|u|} \dot{\psi}_{j, k}(t) \psi_{j, k}(t+|u|) .
\end{aligned}
$$


Sob a suposição $4.2, \operatorname{Var}\left\{d_{j, k}^{(\psi)}\right\} \rightarrow \eta_{j, k}^{(\psi)}$, quando $T \rightarrow \infty$.

Para a ondaleta de Haar, as $d_{j, k}^{(H)}$ são dadas por

$$
d_{j, k}^{(\psi)}=2^{-j / 2}\left[\sum_{t=k 2^{\jmath}}^{2^{\jmath}(k+1 / 2)-1} X(t)-\sum_{t=2^{\jmath}(k+1 / 2)}^{2^{\jmath}(k+1)-1} X(t)\right],
$$

que são diferenças re-escaladas de médias locais de $X(t)$.

Agora vamos investigar a estrutura de covariância da transformada discreta de ondaletas. O próximo resultado é imediato.

Teorema 4.2: Sob a suposição $4.1 \mathrm{e}$

$$
\sum_{u=-\infty}^{\infty}|\gamma(u)|<\infty
$$

definindo

$$
\eta_{\left(j, j^{\prime}\right),\left(k, k^{\prime}\right)}^{(\psi)}=\sum_{u=-\infty}^{\infty} \sum_{t=0}^{\infty} \gamma(u) \psi_{j, k}\left(t+|u| I_{\{u>0\}}\right) \psi_{j^{\prime}, k^{\prime}}\left(t+|u| I_{\{u \leq 0\}}\right)
$$

para $j, j^{\prime}, k, k^{\prime} \in Z$, temos

i) $E\left\{d_{j, k}^{(\psi)} d_{j^{\prime}, k^{\prime}}^{(\psi)}\right\} \rightarrow \eta_{\left(j, j^{\prime}\right),\left(k, k^{\prime}\right)}^{(\psi)}$, quando $T \rightarrow \infty$;

ii) se $j=j^{\prime}, k=k^{\prime}$, então $\eta_{\left(j, j^{\prime}\right),\left(k, k^{\prime}\right)}^{(\psi)}=\eta_{j, k}^{(\psi)}$.

Observação: $\eta_{\left(j, j^{\prime}\right),\left(k, k^{\prime}\right)}^{(\psi)}$ é chamada covariância assintótica das transformadas de ondaletas com respeito a $\psi$.

Teorema 4.3: Sob as suposições 4.1 e 4.2, temos

$$
E\left\{d_{j, k}^{(\psi)} d_{j^{\prime}, k^{\prime}}^{(\psi)}\right\}=O(1), \quad \text { quando } \quad T \rightarrow \infty
$$

para $T=2^{M}$ e $\forall(j, k),\left(j^{\prime}, k^{\prime}\right)$.

\section{Demonstração:}


Usando (4.4),

$$
\begin{aligned}
E\left\{d_{j, k}^{(\psi)} d_{j^{\prime}, k^{\prime}}^{(\psi)}\right\} & =\sum_{t, s=0}^{T-1} \gamma(t-s) \psi_{j, k}(t) \psi_{j^{\prime}, k^{\prime}}(s) \\
& =\sum_{u=-(T-1)}^{T-1} \gamma(u) \sum_{t=0}^{T-1-|u|} \psi_{j, k}\left(t+|u| I_{\{u>0\}}\right) \psi_{j^{\prime}, k^{\prime}}\left(t+|u| I_{\{u \leq 0\}}\right) .
\end{aligned}
$$

Em seguida, com $u>0$, (o caso de $u<0$ segue-se da mesma maneira),

$$
\begin{aligned}
& \left|\sum_{t} \psi_{j, k}(t+u) \psi_{j^{\prime}, k^{\prime}}(t)-\sum_{t} \psi_{j, k}(t) \psi_{j^{\prime}, k^{\prime}}(t)\right| \\
& \quad \leq \sum_{t}\left|\psi_{j^{\prime}, k^{\prime}}(t)\right|\left|\psi_{j, k}(t+u)-\psi_{j, k}(t)\right| \\
& \quad \leq 2^{-j^{\prime} / 2} A \sum_{t} \mid \psi_{j, k}(t+u)-\psi_{j, k}(t+u-1)+\psi_{j, k}(t+u-1)-\psi_{j, k}(t+u-2)+ \\
& \quad+\psi_{j, k}(t+u-2)-\cdots+\psi_{j, k}(t+1)-\psi_{j, k}(t) \mid \\
& \quad \leq 2^{-j^{\prime} / 2} A \sum_{t}\left\{\left|\psi_{j, k}(t+u)-\psi_{j, k}(t+u-1)\right|+\left|\psi_{j, k}(t+u-1)-\psi_{j, k}(t+u-2)\right|+\right. \\
& \left.\quad+\cdots+\left|\psi_{j, k}(t+1)-\psi_{j, k}(t)\right|\right\} \\
& \quad \leq 2^{-j^{\prime} / 2} A 2^{-j / 2} V|u| \\
& \quad=2^{-\left(j^{\prime}+j\right) / 2} A V|u|,
\end{aligned}
$$

onde $V$ é a variação de $\psi$. Portanto,

$$
\begin{aligned}
E\left\{d_{j, k}^{(\psi)} d_{j^{\prime}, k^{\prime}}^{(\psi)}\right\} & =\sum_{u=-(T-1)}^{T-1} \gamma(u) \sum_{t=0}^{T-1-|u|} \psi_{j, k}\left(t+|u| I_{\{u>0\}}\right) \psi_{j^{\prime}, k^{\prime}}\left(t+|u| I_{\{u \leq 0\}}\right) \\
& =\sum_{u=-(T-1)}^{T-1} \gamma(u) \sum_{t=0}^{T-1-|u|} \psi_{j, k}(t) \psi_{j^{\prime}, k^{\prime}}(t)+\epsilon_{T} \\
& =O(1),
\end{aligned}
$$

quando $T \rightarrow \infty$, pois pela suposição 4.2 , temos

$$
\left|\sum_{u} \gamma(u) \sum_{t} \psi_{j, k}(t) \psi_{j^{\prime}, k^{\prime}}(t)\right|
$$




$$
\begin{aligned}
& \leq \sum_{u}|\gamma(u)| \sum_{t}\left|\psi_{j, k}(t) \psi_{j^{\prime}, k^{\prime}}(t)\right| \\
& \leq \sum_{u}|\gamma(u)| 2^{j / 2} A 2^{j^{\prime} / 2} A \mid\left[\text { support }_{j, k}\right] \cap\left[\text { support }_{j^{\prime}, k^{\prime}}\right] \mid \\
& \leq \sum_{u}|\gamma(u)| 2^{\left(j+j^{\prime}\right) / 2} A^{2} \mid\left[\text { support }_{j, k}\right] \cap\left[\text { support }_{j^{\prime}, k^{\prime}}\right] \mid<\infty,
\end{aligned}
$$

$\mathrm{e}$

$$
\begin{aligned}
\left|\epsilon_{T}\right| & \leq \sum_{u=-(T-1)}^{T-1}|\gamma(u)| 2^{-\left(j^{\prime}+j\right) / 2} A V|u| \\
& \leq 2^{-\left(j^{\prime}+j\right) / 2} A V \sum_{u=-(T-1)}^{T-1}|u \| \gamma(u)|<\infty .
\end{aligned}
$$

Pelo Teorema 4.3 vemos que a covariância assintótica das transformadas finitas de ondaletas em dois pares distintos $(j, k)$ e $\left(j^{\prime}, k^{\prime}\right)$ não é necessariamente zero, diferente do caso da transformada de Fourier, onde as covariâncias são assintoticamente zero, sob algumas suposições. Entretanto, em algumas situações essa propriedade pode valer.

Teorema 4.4: Se $\psi(t)$ tem suporte $\left[K_{1}, K_{2}\right]$, onde $K_{1} \geq 0$ e $K_{2} \geq 0$ e $X(t)$ é uma série temporal estacionária discreta, com média zero, $\gamma(u) \neq 0$ para $|u| \leq U, U<<T$, então, $E\left(d_{j, k_{1}}^{(\psi)} d_{j, k_{2}}^{(\psi)}\right)=0$ para $\left|k_{1}-k_{2}\right|>K_{2}-K_{1}+\frac{U}{2^{\jmath}}$, $j=1, \cdots, M$ e $k=0,1, \cdots,\left(2^{M-j}-1\right)$.

Isto é, escolhendo uma ondaleta com valor grande para $K_{2}$ e valor pequeno para $K_{1}$, poucos coeficientes de ondaletas satisfazem a condição $\left|k_{1}-k_{2}\right|>$ $K_{2}-K_{1}+\frac{U}{2^{\jmath}}$, e então as $d_{j, k}^{(\psi)}$ são não correlacionadas somente sobre um conjunto menor.

\section{Demonstração:}

Temos que

$$
E\left(d_{j, k_{1}}^{(\psi)} d_{j, k_{2}}^{(\psi)}\right)=\sum_{u=-(T-1)}^{T-1} \gamma(u) \sum_{t=0}^{T-1-|u|} \psi_{j, k_{1}}\left(t+|u| I_{\{u>0\}}\right) \psi_{j, k_{2}}\left(t+|u| I_{\{u \leq 0\}}\right)
$$

e então,

$$
\left[\text { support }_{j, k_{1}}\right]=\left[2^{j}\left(K_{1}+k_{1}\right)-|u| I_{\{u>0\}} ; 2^{j}\left(K_{2}+k_{1}\right)-|u| I_{\{u>0\}}\right]
$$




$$
\left[\text { support } \psi_{j, k_{2}}\right]=\left[2^{j}\left(K_{1}+k_{2}\right)-|u| I_{\{u \leq 0\}} ; 2^{j}\left(K_{2}+k_{2}\right)-|u| I_{\{u \leq 0\}}\right] .
$$

Segue-se que

$$
\left[\text { support } \psi_{j, k_{1}}\right] \cap\left[\text { support }_{j, k_{2}}\right]
$$

é vazio se $\left|k_{1}-k_{2}\right|>K_{2}-K_{1}+\frac{U}{2^{3}}$.

Agora vamos ver um teorema limite central para a transformada de ondaletas. A demonstração baseia-se em Hannan(1973).

Teorema 4.5: Suponha que $X(t)$ seja estritamente estacionário de média zero, $E|X(t)|^{2}<\infty$ e seja $\mathcal{M}_{t}$ a $\sigma$-álgebra gerada por $(X(j), j \leq t)$. Defina

$$
\alpha_{j}=\left[E\left\{E\left(X(t) / \mathcal{M}_{t-j}\right)-E\left(X(t) / \mathcal{M}_{t-j-1}\right)\right\}^{2}\right]^{1 / 2}, \quad j \geq 0 .
$$

Assumimos que $\mathcal{M}_{-\infty}$ é trivial e que $\sum_{j=0}^{\infty} \alpha_{j}<\infty$.

(i) Para $\left(j_{1}, k_{1}\right), \cdots,\left(j_{p}, k_{p}\right)$ e $m, n=1, \cdots, p$, suponha que

$$
E\left\{d_{j_{m}, k_{m}}^{(\psi)} d_{j_{n}, k_{n}}^{(\psi)}\right\} \rightarrow \eta_{\left(j_{m}, j_{n}\right),\left(k_{m}, k_{n}\right)}^{(\psi)}, \quad \text { quando } \quad T \rightarrow \infty .
$$

Então,

$$
\Phi \stackrel{\mathcal{D}}{\rightarrow} \mathcal{N}_{p}(0, \Delta)
$$

onde

$$
\Delta=\left(\eta_{\left(j_{m}, j_{n}\right),\left(k_{m}, k_{n}\right)}^{(\psi)}\right)
$$

e

$$
\Phi=\left(d_{j_{1}, k_{1}}^{(\psi)}, \cdots, d_{j_{p}, k_{p}}^{(\psi)}\right)^{\prime} .
$$

(ii) Em particular, temos

$$
d_{j, k}^{(\psi)} \stackrel{\mathcal{D}}{\rightarrow} \mathcal{N}\left(0, \eta_{j, k}^{(\psi)}\right) .
$$

\section{Demonstração:}


(i) Seja $Y^{(T)}(t)=\sqrt{T} \psi_{j, k}(t)$ e $d^{2}(T)=\sum_{t=0}^{T-1}\left\{Y^{(T)}(t)\right\}^{2}$. Então,

$$
\begin{aligned}
d^{2}(T) & =\sum_{t=0}^{T-1}\left\{Y^{(T)}(t)\right\}^{2} \\
& =T \sum_{t=0}^{T-1}\left[\psi_{j, k}(t)\right]^{2} \\
& =T+O(1),
\end{aligned}
$$

pois

$$
\sum_{t=0}^{T-1}\left[\psi_{j, k}(t)\right]^{2}=1+O\left(T^{-1}\right) .
$$

Agora temos,

. $\lim _{T \rightarrow \infty} d^{2}(T)=\infty$;

. $\lim _{T \rightarrow \infty} \frac{\left|Y^{(T)}(T)\right|}{d(T)}=\lim _{T \rightarrow \infty} \frac{\sqrt{T}\left|\psi_{, k}(T)\right|}{\sqrt{T}}=0$, pois $\mid$ support $\psi_{j, k} \mid<\infty$;

$. \lim _{T \rightarrow \infty} \frac{\left\{\sum_{m=1}^{T-t} Y^{(T)}(m) Y^{(T)}(m+t)\right\}}{d^{2}(T)}=\lim _{T \rightarrow \infty} \frac{T\left\{\sum_{s=0}^{T-1-t} \psi_{j, k}(s) \psi_{j, k}(s+t)\right\}}{T}=\rho(t)$.

Portanto temos,

$$
\rho(t)=\lim _{T \rightarrow \infty}\left\{\sum_{s=0}^{T-1-t} \psi_{j, k}(s) \psi_{j, k}(s+t)\right\} .
$$

Agora,

$$
\begin{aligned}
\eta_{j, k}^{(\psi)} & =\sum_{t=0}^{\infty} \sum_{s=0}^{\infty} \gamma(t-s) \psi_{j, k}(t) \psi_{j, k}(s) \\
& =\lim _{T \rightarrow \infty} \sum_{t=0}^{T-1} \sum_{s=0}^{T-1} \gamma(t-s) \psi_{j, k}(s) \psi_{j, k}(t) \\
& =\lim _{T \rightarrow \infty} \sum_{u=-(T-1)}^{T-1} \gamma(u) \sum_{s=0}^{T-1-|u|} \psi_{j, k}(s) \psi_{j, k}(s+|u|) \\
& =\sum_{u=-\infty}^{\infty} \gamma(u)\left\{\lim _{T \rightarrow \infty} \sum_{s} \psi_{j, k}(s) \psi_{j, k}(s+|u|)\right\} \\
& =\sum_{u=-\infty}^{\infty} \gamma(u) \rho(|u|) .
\end{aligned}
$$


Portanto, (i) pode ser provada como no Teorema 1(i) de Hannan (1973).

(ii) Imediata.

Observação: O teorema 4.5 é provado sob uma forma de "strong mixing condition", isto é, uma independência assintótica.

Outro teorema limite central pode ser provado, sob uma forma diferente de "mixing assumption", baseado em cumulantes. Defina

$$
C_{x}\left(u_{1}, \cdots, u_{k-1}\right)=C u m\left\{X\left(t+u_{1}\right), \cdots, X\left(t+u_{k-1}\right), X(t)\right\}
$$

como o cumulante de ordem $k$ de $X(t), u_{1}, \cdots, u_{k-1}=0, \pm 1, \cdots, k=2,3 \cdots$ e $C_{x}\left(u_{1}\right)=\gamma\left(u_{1}\right)$.

Sob a suposição que

$$
\sum_{u_{1}} \cdots \sum_{u_{k-1}}\left|u_{j}\right|\left|C_{x}\left(u_{1}, \cdots, u_{k-1}\right)\right|<\infty,
$$

pode-se provar que $d_{j, k}^{(\psi)}$ é assintoticamente normal com média zero e variância $\sum_{u} \gamma(u)$. Note que $\sum_{u} \gamma(u)=2 \pi f(0)$, onde $f(\lambda)$ é o espectro de Fourier de $X(t)$. A demonstração é similar a de Brillinger (1996).

\subsection{O Periodograma de Ondaletas}

Temos uma sequência observada de valores $X(0), \cdots, X(T-1)$ e baseados nesses valores queremos estimar $\eta_{j, k}^{(\psi)}$.

Em primeiro lugar, calculamos a transformada finita de ondaletas com respeito a $\psi$ :

$$
d_{j, k}^{(\psi)}=\sum_{t=0}^{T-1} X(t) \psi_{j, k}(t) .
$$

Provamos que $d_{j, k}^{(\psi)}$ é assintoticamente normal $\mathcal{N}\left(0, \eta_{j, k}^{(\psi)}\right)$, daí segue-se que $\eta_{j, k}^{(\psi)}$ pode ser estimado pela estatística

$$
I_{j, k}^{(\psi)}=\left(d_{j, k}^{(\psi)}\right)^{2}=\left[\sum_{t=0}^{T-1} \mathrm{X}(t) \psi_{j, k}(t)\right]^{2} .
$$


A estatística $I_{j, k}^{(\psi)}$ de (4.12) é chamada o periodograma de segunda ordem de ondaletas, ou simplesmente o periodograma de ondaletas dos valores $X(0), \cdots$, $X(T-1)$.

Observação: Vamos definir a " energia" numa série temporal como $\sum_{t=0}^{T-1} X^{2}(t)$, então, usando o teorema de Parseval, podemos decompor a energia numa série temporal com respeito a escala $j$ e tempo $k$ por $I_{j, k}^{(\psi)}$ para $j=1, \cdots, M$, $k=0,1, \cdots, 2^{(M-j)}-1$ e $T=2^{M}$.

No Teorema 4.1, mostramos que $I_{j, k}^{(\psi)}$ é assintoticamente não viciado. Também temos o resultado seguinte.

Teorema 4.6: Sob as suposições 4.1 e 4.2,

$$
E\left[I_{j, k}^{(\psi)}\right]=\eta_{j, k}^{(\psi)}+O\left(T^{-1}\right) .
$$

\section{Demonstração:}

$\operatorname{Temos} I_{j, k}^{(\psi)}=\left[d_{j, k}^{(\psi)}\right]^{2}, \operatorname{logo}$

$$
\begin{aligned}
E\left\{I_{j, k}^{(\psi)}\right\} & =\sum_{t=0}^{T-1} \sum_{s=0}^{T-1} \gamma(t-s) \psi_{j, k}(t) \psi_{j, k}(s) \\
& =\sum_{u=-(T-1)}^{T-1} \gamma(u) \sum_{t=0}^{T-1-|u|} \psi_{j, k}(t) \psi_{j, k}(t+|u|) \\
& =\sum_{u=-\infty}^{\infty} \gamma(u) \sum_{t=0}^{\infty} \psi_{j, k}(t) \psi_{j, k}(t+|u|)+\eta_{T} \\
& =\eta_{j, k}^{(\psi)}+\eta_{T},
\end{aligned}
$$

onde

$$
\begin{aligned}
\left|\eta_{T}\right|= & \mid \sum_{u=T}^{\infty} \gamma(u) \sum_{t=0}^{\infty} \psi_{j, k}(t) \psi_{j, k}(t+|u|)+\sum_{u=-\infty}^{-T} \gamma(u) \sum_{t=0}^{\infty} \psi_{j, k}(t) \psi_{j, k}(t+|u|) \\
& +\sum_{u=-(T-1)}^{T-1} \gamma(u) \sum_{t=T}^{\infty} \psi_{j, k}(t-|u|) \psi_{j, k}(t) \mid \\
\leq & \left|\sum_{u=T}^{\infty} \gamma(u) \sum_{t=0}^{\infty} \psi_{j, k}(t) \psi_{j, k}(t+|u|)\right|+\left|\sum_{u=-\infty}^{-T} \gamma(u) \sum_{t=0}^{\infty} \psi_{j, k}(t) \psi_{j, k}(t+|u|)\right|
\end{aligned}
$$




$$
\begin{aligned}
& +\left|\sum_{u=-(T-1)}^{T-1} \gamma(u) \sum_{t=T}^{\infty} \psi_{j, k}(t-|u|) \psi_{j, k}(t)\right| \\
& \leq\left|S_{1}\right|+\left|S_{2}\right|+\left|S_{3}\right| .
\end{aligned}
$$

Agora,

$$
\begin{aligned}
\left|S_{1}\right| & \leq \sum_{u=T}^{\infty} \frac{|u|}{T}|\gamma(u)| \sum_{t=0}^{\infty}\left|\psi_{j, k}(t) \psi_{j, k}(t+|u|)\right| \\
& \leq \frac{1}{T} \sum_{u=-\infty}^{\infty}|u \| \gamma(u)| \sum_{t=0}^{\infty}\left|\psi_{j, k}(t) \psi_{j, k-2-\jmath}\right| u|(t)| \\
& \leq \frac{1}{T} \sum_{-\infty}^{\infty}|u \| \gamma(u)| 2^{-j} A \mid\left[\text { support }_{j, k}\right] \cap\left[\text { support }_{j, k-2^{-\jmath}|u|}\right] \mid .
\end{aligned}
$$

Portanto, $S_{1}=O\left(T^{-1}\right)$.

Da mesma maneira, $S_{2}=O\left(T^{-1}\right)$.

Agora,

$$
\begin{aligned}
& \left|S_{3}\right| \leq \sum_{u=-(T-1)}^{T-1}|\gamma(u)| \sum_{t=T}^{\infty} \frac{|t|}{T}\left|\psi_{j, k}(t-|u|) \psi_{j, k}(t)\right| \\
& \leq \frac{1}{T} \sum_{u=-\infty}^{\infty}|\gamma(u)| \sum_{t=T}^{\infty}|t|\left|\psi_{j, k+2^{-\jmath}|u|}(t) \psi_{j, k}(t)\right| \\
& \leq \frac{1}{T} \sum_{u=-\infty}^{\infty}|\gamma(u)| 2^{-j} A \mid\left[\text { support }_{j, k+2^{-\jmath}|u|}\right] \cap\left[\text { support }_{j, k}\right] \mid M_{\psi} \text {, }
\end{aligned}
$$

onde $-\infty<M_{\psi}=\sup _{j, k}\left\{\left[\right.\right.$ support $\left._{j, k+2^{-\jmath}|u|}\right] \cap\left[\right.$ support $\left.\left._{j, k}\right]\right\}<\infty$. Portanto, $S_{3}=O\left(T^{-1}\right)$.

A estrutura de covariância do periodograma é dada a seguir.

Teorem 4.7: Sob as suposições 4.1 e 4.2 e

$$
\sum_{l, m, n=-\infty}^{\infty}\left|C_{x}(l, m, n)\right|<\infty
$$


onde $C_{x}(l, m, n)=C u m\{X(0), X(l), X(m), X(n)\}$ é o cumulante de quarta ordem de $X(t)$, temos

$$
\operatorname{Cov}\left\{I_{j, k}^{(\psi)}, I_{j^{\prime}, k^{\prime}}^{(\psi)}\right\}=2\left\{\eta_{\left(j, j^{\prime}\right),\left(k, k^{\prime}\right)}^{(\psi)}\right\}^{2}+O(1), \quad \text { quando } \quad T \rightarrow \infty
$$

\section{Demonstração:}

$$
\begin{aligned}
I_{j, k}^{(\psi)} I_{j^{\prime}, k^{\prime}}^{(\psi)} & =\left(d_{j, k}^{(\psi)}\right)^{2}\left(d_{j^{\prime}, k^{\prime}}^{(\psi)}\right)^{2} \\
& =\left[\sum_{t_{1}=0}^{T-1} X\left(t_{1}\right) \psi_{j, k}\left(t_{1}\right)\right]^{2}\left[\sum_{t_{2}=0}^{T-1} X\left(t_{2}\right) \psi_{j^{\prime}, k^{\prime}}\left(t_{2}\right)\right]^{2} \\
& =\sum_{t_{1}, s_{1}, t_{2}, s_{2}=0}^{T-1} X\left(t_{1}\right) X\left(s_{1}\right) X\left(t_{2}\right) X\left(s_{2}\right) \psi_{j, k}\left(t_{1}\right) \psi_{j, k}\left(s_{1}\right) \psi_{j^{\prime}, k^{\prime}}\left(t_{2}\right) \psi_{j^{\prime}, k^{\prime}}\left(s_{2}\right),
\end{aligned}
$$

e daí,

$$
E\left\{I_{j, k}^{(\psi)} I_{j^{\prime}, k^{\prime}}^{(\psi)}\right\}=\sum_{t_{1}, s_{1}, t_{2}, s_{2}=0}^{T-1} E\left\{X\left(t_{1}\right) X\left(s_{1}\right) X\left(t_{2}\right) X\left(s_{2}\right)\right\} \psi_{j, k}\left(t_{1}\right) \psi_{j, k}\left(s_{1}\right) \psi_{j^{\prime}, k^{\prime}}\left(t_{2}\right) \psi_{j^{\prime}, k^{\prime}}\left(s_{2}\right) .
$$

Baseado em Hannan (1970, pag.23), temos,

$$
\begin{aligned}
& E\left\{I_{j, k}^{(\psi)} I_{j^{\prime}, k^{\prime}}^{(\psi)}\right\}=\sum_{t_{1}, s_{1}, t_{2}, s_{2}=0}^{T-1}\left\{\gamma\left(t_{1}-s_{1}\right) \gamma\left(t_{2}-s_{2}\right)+\gamma\left(t_{1}-s_{2}\right) \gamma\left(s_{1}-t_{2}\right)+\gamma\left(s_{1}-s_{2}\right) .\right. \\
& \left.\gamma\left(t_{1}-t_{2}\right)+C_{x}\left(s_{1}-t_{1}, t_{2}-t_{1}, s_{2}-t_{1}\right)\right\} \psi_{j, k}\left(t_{1}\right) \psi_{j, k}\left(s_{1}\right) \psi_{j^{\prime}, k^{\prime}}\left(t_{2}\right) \psi_{j^{\prime}, k^{\prime}}\left(s_{2}\right) \\
& =S_{1}+S_{2}+S_{3}+S_{4} \text {. } \\
& S_{1}=\sum_{t_{1}, s_{1}, t_{2}, s_{2}=0}^{T-1} \gamma\left(t_{1}-s_{1}\right) \gamma\left(t_{2}-s_{2}\right) \psi_{j, k}\left(t_{1}\right) \psi_{j, k}\left(s_{1}\right) \psi_{j^{\prime}, k^{\prime}}\left(t_{2}\right) \psi_{j^{\prime}, k^{\prime}}\left(s_{2}\right) \\
& =\left\{\sum_{t_{1}, s_{1}=0}^{T-1} \gamma\left(t_{1}-s_{1}\right) \psi_{j, k}\left(t_{1}\right) \psi_{j, k}\left(s_{1}\right)\right\}\left\{\sum_{t_{2}, s_{2}=0}^{T-1} \gamma\left(t_{2}-s_{2}\right) \psi_{j^{\prime}, k^{\prime}}\left(t_{2}\right) \psi_{j^{\prime}, k^{\prime}}\left(s_{2}\right)\right\} \\
& =E\left(I_{j, k}^{(\psi)}\right) E\left(I_{j^{\prime}, k^{\prime}}^{(\psi)}\right) \text {. }
\end{aligned}
$$




$$
\begin{aligned}
S_{2} & =\sum_{t_{1}, s_{1}, t_{2}, s_{2}=0}^{T-1} \gamma\left(t_{1}-s_{2}\right) \gamma\left(s_{1}-t_{2}\right) \psi_{j, k}\left(t_{1}\right) \psi_{j, k}\left(s_{1}\right) \psi_{j^{\prime}, k^{\prime}}\left(t_{2}\right) \psi_{j^{\prime}, k^{\prime}}\left(s_{2}\right) \\
& =\left\{\sum_{t_{1}, s_{2}=0}^{T-1} \gamma\left(t_{1}-s_{2}\right) \psi_{j, k}\left(t_{1}\right) \psi_{j^{\prime}, k^{\prime}}\left(s_{2}\right)\right\}\left\{\sum_{t_{2}, s_{1}=0}^{T-1} \gamma\left(s_{1}-t_{2}\right) \psi_{j, k}\left(s_{1}\right) \psi_{j^{\prime}, k^{\prime}}\left(t_{2}\right)\right\} \\
& \rightarrow\left\{\eta_{\left(j, j^{\prime}\right),\left(k, k^{\prime}\right)}^{(\psi)}\right\}^{2}, \quad \text { quando } \quad T \rightarrow \infty . \\
S_{3} & =\sum_{t_{1}, s_{1}, t_{2}, s_{2}=0}^{T-1} \gamma\left(s_{1}-s_{2}\right) \gamma\left(t_{1}-t_{2}\right) \psi_{j, k}\left(t_{1}\right) \psi_{j, k}\left(s_{1}\right) \psi_{j^{\prime}, k^{\prime}}\left(t_{2}\right) \psi_{j^{\prime}, k^{\prime}}\left(s_{2}\right) \\
& =\left\{\sum_{s_{1}, s_{2}=0}^{T-1} \gamma\left(s_{1}-s_{2}\right) \psi_{j, k}\left(s_{1}\right) \psi_{j^{\prime}, k^{\prime}}\left(s_{2}\right)\right\}\left\{\sum_{t_{1}, t_{2}=0}^{T-1} \gamma\left(t_{1}-t_{2}\right) \psi_{j, k}\left(t_{1}\right) \psi_{j^{\prime}, k^{\prime}}\left(t_{2}\right)\right\} \\
& \rightarrow\left\{\eta_{\left(j, j^{\prime}\right),\left(k, k^{\prime}\right)}^{(\psi)}\right\}^{2}, \quad \text { quando } T \rightarrow \infty .
\end{aligned}
$$

Finalmente,

$$
\left|S_{4}\right|=\left|\sum_{t_{1}, s_{1}, t_{2}, s_{2}=0}^{T-1} C_{x}\left(s_{1}-t_{1}, t_{2}-t_{1}, s_{2}-t_{1}\right) \psi_{j, k}\left(t_{1}\right) \psi_{j, k}\left(s_{1}\right) \psi_{j^{\prime}, k^{\prime}}\left(t_{2}\right) \psi_{j^{\prime}, k^{\prime}}\left(s_{2}\right)\right|<\infty
$$

se (4.7) estiver satisfeita. Então $S_{4}=O(1)$.

Portanto,

$$
\operatorname{Cov}\left(I_{j, k}^{(\psi)}, I_{j^{\prime}, k^{\prime}}^{(\psi)}\right)=2\left\{\eta_{\left(j, j^{\prime}\right),\left(k, k^{\prime}\right)}^{(\psi)}\right\}^{2}+O(1), \quad \text { quando } \quad T \rightarrow \infty .
$$

Em particular, se $j=j^{\prime}, k=k^{\prime}$, obtemos a variância

$$
\operatorname{Var}\left[I_{j, k}^{(\psi)}\right]=2\left\{\eta_{j, k}^{(\psi)}\right\}^{2}+O(1)
$$

Observação: O Teorema 4.7 mostra que o periodograma de ondaletas é um estimador não consistente, dado que a variância não decresce para zero, quando $T \rightarrow \infty$.

A distribuição assintótica do periodograma de ondaletas é obtida imediatamente a partir do Teorema 4.5. 
Teorema 4.8: Sob as suposições do Teorema 4.5, $I_{j, k}^{(\psi)}$ é uma variável assintoticamente $\eta_{j, k}^{(\psi)} \chi_{1}^{2}$.

\section{Demonstração:}

Como $d_{j, k}^{(\psi)}$ é assintoticamente $\mathcal{N}\left(0, \eta_{j, k}^{(\psi)}\right)$, então

$$
\frac{d_{j, k}^{(\psi)}}{\left[\eta_{j, k}^{(\psi)}\right]^{1 / 2}}
$$

é assintoticamente $\mathcal{N}(0,1)$, portanto seu quadrado é assintoticamente $\chi_{1}^{2}$.

Lembramos que o periodograma de Fourier $I(\lambda)=\frac{1}{2 \pi T}\left|\sum_{t=0}^{T-1} X(t) e^{-i \lambda t}\right|^{2}$ tem, para cada frequência $-\pi<\lambda<\pi, \lambda \neq 0$, uma distribuição assintoticamente $f(\lambda) \chi_{2}^{2}$, onde $f(\lambda)$ é o (Fourier) espectro de $X(t)$. O Teorema 4.8 nos diz que para cada $j=1, \cdots, M$ e cada $k=0, \cdots,\left(2^{(M-j)}-1\right), I_{j, k}^{(\psi)}$ é uma variável assintoticamente $\eta_{j, k}^{(\psi)} \chi_{1}^{2}$. Portanto, intervalos de confiança aproximada para $\eta_{j, k}^{(\psi)}$ podem ser construídos, do mesmo modo que são para $f(\lambda)$.

\subsection{O Escalograma}

Em (4.6) definimos o periodograma de ondaletas com respeito a $\psi$. Note que $I_{j, k}^{(\psi)}$ é definido por cada $(j, k), j=1, \cdots, M$ e $k=0, \cdots, 2^{(M-j)}-1$, $T=2^{M}$.

Podemos definir também o escalograma na escala $j$ como sendo

$$
S^{(\psi)}(j)=\sum_{k=0}^{2^{(M-\jmath)}-1}\left[d_{j, k}^{(\psi)}\right]^{2}=\sum_{k=0}^{2^{(M-\jmath)}-1} I_{j, k}^{(\psi)}, \quad j=1, \cdots, M .
$$

Podemos pensar $S^{(\psi)}(j)$ como a energia dos coeficientes de ondaletas na escala (ou nível) $j$. Ver Scargle (1993) e Arino e Vidakovic (1995).

Teorema 4.9: Sob as suposições do Teorema 4.7, temos

$$
E\left\{S^{(\psi)}(j)\right\}=\eta_{j, \cdot}^{(\psi)}+O\left(2^{(M-j)} T^{-1}\right)
$$

$\mathrm{e}$

$$
\operatorname{Cov}\left\{S^{(\psi)}(j), S^{(\psi)}\left(j^{\prime}\right)\right\}=2 \sum_{k=0}^{2^{(M-\jmath)}-1} \sum_{k^{\prime}=0}^{2^{\left(M-j^{\prime}\right)}-1}\left\{\eta_{\left(j, j^{\prime}\right),\left(k, k^{\prime}\right)}^{(\psi)}\right\}^{2}+O(1),
$$


onde

$$
\eta_{j, \cdot}^{(\psi)}=\sum_{k=0}^{2^{(M-\jmath)}-1} \eta_{j, k}^{(\psi)} .
$$

\section{Demonstração:}

Temos

$$
E\left\{S^{(\psi)}(j)\right\}=\sum_{k} E\left\{I_{j, k}^{(\psi)}\right\}=\sum_{k} \eta_{j, k}^{(\psi)}+O\left(2^{(M-j)} T^{-1}\right)=\eta_{j, \cdot}^{(\psi)}+O\left(2^{(M-j)} T^{-1}\right),
$$

$\mathrm{e}$

$$
\begin{aligned}
\operatorname{Cov}\left\{S^{(\psi)}(j), S^{(\psi)}\left(j^{\prime}\right)\right\} & =E\left\{S^{(\psi)}(j) S^{(\psi)}\left(j^{\prime}\right)\right\}-E\left\{S^{(\psi)}(j)\right\} E\left\{S^{(\psi)}\left(j^{\prime}\right)\right\} \\
& =E\left\{\sum_{k} I_{j, k}^{(\psi)} \sum_{k^{\prime}} I_{j^{\prime}, k^{\prime}}^{(\psi)}\right\}-E\left\{\sum_{k} I_{j, k}^{(\psi)}\right\} E\left\{\sum_{k^{\prime}} I_{j^{\prime}, k^{\prime}}^{(\psi)}\right\} \\
& =\sum_{k} \sum_{k^{\prime}}\left\{E\left(I_{j, k}^{(\psi)} I_{j^{\prime}, k^{\prime}}^{(\psi)}\right)-E\left(I_{j, k}^{(\psi)}\right) E\left(I_{j^{\prime}, k^{\prime}}^{(\psi)}\right)\right\} \\
& =\sum_{k} \sum_{k^{\prime}} \operatorname{Cov}\left(I_{j, k}^{(\psi)}, I_{j^{\prime}, k^{\prime}}^{(\psi)}\right) \\
& =\sum_{k} \sum_{k^{\prime}} 2\left\{\eta_{\left(j, j^{\prime}\right),\left(k, k^{\prime}\right)}^{(\psi)}\right\}^{2}+O(1) .
\end{aligned}
$$

$\eta_{j, \cdot}^{(\psi)}$ pode ser visto como a energia espectral associada com a escala $j$ e $S^{(\psi)}(j)$ como seu estimador.

Baseado no Teorema 4.8, $I_{j, k}^{(\psi)}$ são distribuídos como uma variável $\eta_{j, k}^{(\psi)} \chi_{1}^{2} \mathrm{e}$ daí, usando as propriedades da distribuição de qui-quadrado, a distribuição de $S^{(\psi)}(j)$ pode ser aproximada por $a \chi_{b}^{2}$, onde $a$ e $b$ são parâmetros a ser determinados.

Teorema 4.10: Sob as suposições dos Teoremas 4.7 e 4.8, $S^{(\psi)}(j)$ é uma variável assintoticamente $a \chi_{b}^{2}$ com

$$
a \approx \frac{\left\{\sum_{k} \sum_{k^{\prime}} \eta_{(j, j),\left(k, k^{\prime}\right)}^{(\psi)}\right\}^{2}}{\eta_{j, \cdot}^{(\psi)}}
$$

$\mathrm{e}$

$$
b \approx \frac{\left\{\eta_{j, \cdot}^{(\psi)}\right\}^{2}}{\sum_{k} \sum_{k^{\prime}}\left\{\eta_{(j, j),\left(k, k^{\prime}\right)}^{(\psi)}\right\}^{2}} .
$$




\section{Demonstração:}

Os dois primeiros momentos da variável $a \chi_{b}^{2}$ são

$$
E\left\{a \chi_{b}^{2}\right\}=a b,
$$

$\mathrm{e}$

$$
\operatorname{Var}\left\{a \chi_{b}^{2}\right\}=2 a^{2} b .
$$

A distribuição de $S^{(\psi)}(j)$ pode ser aproximada por $a \chi_{b}^{2}$ pois $I_{j, k}^{(\psi)}$ é uma variável assintoticamente qui-quadrada. Então as constantes $a$ e $b$ podem ser calculadas a partir de

e

$$
b \approx \frac{2\left\{E\left(S^{(\psi)}(j)\right)\right\}^{2}}{\operatorname{Var}\left\{S^{(\psi)}(j)\right\}}
$$

$$
a \approx \frac{E\left\{S^{(\psi)}(j)\right\}}{b}
$$

as quais fornecem os parâmetros da distribuição aproximada de qui-quadrado em termos dos dois primeiros momentos de $S^{(\psi)}(j)$.

Agora a partir de (4.9) e (4.10) obtemos (4.12) e (4.13).

Como uma ilustração, consideremos uma série temporal harmônica dada pela soma de duas séries periódicas com períodos de 5 e 24 ,

para $t=0, \pm 1, \cdots, 2^{10}-1$.

$$
X(t)=\sin \left(\frac{\pi}{12} t\right)+2 \sin \left(\frac{2 \pi}{5} t\right)
$$

Observação: Esta série não satisfaz as suposições 4.1 e 4.2, mas calculamos o escalograma do mesmo modo que calculamos o periodograma usual de uma série com espectro misto, para detectar periodicidades.

Parte desta série $X(t)$ é apresentada na figura B.4 e o escalograma, como definido em (4.8), é mostrado na figura B.5. A presença de um pico no escalograma de uma série temporal num nível baixo $j$ significa que um ciclo de frequência alta (ou período curto) está presente na série. Por outro lado, quando existe um pico num nível alto $j$, isto indica que um ciclo de frequência baixa (ou período longo) está presente na série. Por exemplo, na figura B.5, existe um pico no nível 2 (provavelmente correspondente ao período de 5 unidades) e um pico no nível 4 (provavelmente correspondente ao período de 24 unidades). Os coeficientes da transformada de ondaletas estão mostrados na figura B.6 e o periodograma na figura B.7. 


\subsection{Encolhimento de Ondaletas}

Encolhimento é básico no trabalho estatístico com ondaletas. O uso de encolhedores (redutores) para melhorar estimadores é comum, ver Thompson (1968) e King (1972). Encolhimento de ondaletas funciona como um suavizador. A idéia é a remoção de coeficientes pequenos de ondaletas que são considerados como ruídos. Este método remove os valores pequenos de $d_{j, k}^{(\psi)}$ e conserva os valores grandes de $d_{j, k}^{(\psi)}$ que podem ser usados para melhorar os estimadores.

O procedimento em geral de encolhimento de ondaletas pode ser descrito em três etapas:

1) use a transformada de ondaletas dos dados (uma série temporal) para obter os coeficientes de ondaletas;

2) trunque os coeficientes de ondaletas através de um limiar;

3) inverta os coeficientes da transformada de ondaletas truncados.

Existem várias maneiras para fazer truncamento. Basicamente, o processo de truncar os coeficientes de ondaletas pode ser dividido em duas etapas: escolhe-se o tipo de suavização (uma função de truncamento $\delta$ ) e escolhe-se o valor de truncamento $(\lambda)$.

Limiarização dura ("Hard thresholding") e limiarização suave (" soft thresholding") são dois tipos de suavização usuais:

$$
\delta_{h}\left(d_{j, k}^{(\psi)}, \lambda\right)=d_{j, k}^{(\psi)} I_{\left\{\left|d_{j, k}^{(\psi)}\right|>\lambda\right\}}, \quad \delta_{s}\left(d_{j, k}^{(\psi)}, \lambda\right)=\operatorname{sign}\left(d_{j, k}^{(\psi)}\right)\left(\left|d_{j, k}^{(\psi)}\right|-\lambda\right)_{+},
$$

respectivamente, onde $\lambda$ é o valor de truncamento.

A segunda etapa é a escolha de um valor de truncamento. A escolha do valor de truncamento, tanto quanto a escolha do tipo de suavização, é um dos temas mais importantes em encolhimento de ondaletas. Donoho e Johnstone $(1994,1995 \mathrm{a}, \mathrm{b})$ e Donoho et al (1995) apresentaram várias propostas no contexto de regressão não-paramétrica, com ruído $N\left(0, \sigma^{2}\right)$. Em particular, o truncamento universal, dado por $\lambda=\sigma \sqrt{2 \log T}$, é de uso frequente. Neste caso, o parâmetro $\lambda$ não depende do nível $j$. Donoho at el (1996) propõem um valor de truncamento $\lambda=\sqrt{j}$ para estimação de densidade. Nason (1995) 
propõe um procedimento muito interessante de seleção do valor de truncamento através de validação cruzada. O valor de truncamento é selecionado minimizando um estimador " cross-validatory" do erro quadrático médio integrado. Gao (1997) propõe o uso dos $\lambda_{j, T}$ dependendo no tamanho da amostra $T$, da ondaleta base e do nível de resolução $j$ para estimar o $\log$-espectro de uma série temporal estacionária Gaussiana. O propósito desse nível de truncamento é fazer o $\log$-espectro reconstruído livre de ruído. Outro procedimento, chamado "SureShrink" foi sugerido, onde o valor de truncamento é selecionado minimizando o estimador não viciado do risco de Stein (SURE) para recuperar uma função desconhecida a partir dos dados amostrais.

Em geral, os métodos de encolhimento de ondaletas apresentam enormes vantagens teóricas, incluindo erro quadrático médio aproximadamente ótimo e adaptação espacial aproximadamente ideal. Para detalhes, ver artigos citados acima.

Uma possibilidade é usar o estimador de encolhimento não-linear

$$
I_{j, k}^{(\psi, \lambda)}=\delta_{h}\left(I_{j, k}^{(\psi)}, \lambda\right)
$$

com finalidade de melhorar o periodograma de ondaleta, onde

$$
\lambda=\lambda_{j, k}=\sigma_{j, k} \log T
$$

ou

$$
\lambda=\lambda_{j}=\sigma_{j} \log T,
$$

$\operatorname{com} \sigma_{j, k}^{2}=\operatorname{Var}\left\{I_{j, k}^{(\psi)}\right\}$ e $\sigma_{j}^{2}=\operatorname{Var}\left\{S^{(\psi)}(j)\right\}$, respectivamente. Basicamente, o uso de $\log T$ no lugar de $\sqrt{\log T}$ tem a ver com as possiveis caudas longas da distribuição (qui-quadrado) de $I_{j, k}^{(\psi)}$ ou $S^{(\psi)}(j)$. Estimadores de $\sigma_{j, k}^{2}$ e $\sigma_{j}^{2}$ são usados em (4.15) e (4.16).

\subsection{Aplicação}

Nesta seção a transformada de ondaletas com respeito à ondaleta de Haar é usada para investigar os componentes espectrais de eletroencefalograma (EEG) durante o estado de sono de bebês cujas mães se abstiveram de bebidas alcoólicas durante a gravidez e bebês cujas mães consumiram (de forma moderada) bebidas alcoólicas durante a gravidez. Os dados foram coletados 
para um estudo sobre efeitos do consumo de álcool maternal no EEG durante o estado de sono de crianças recém-nascidas. A descrição detalhada do planejamento de estudo, as metodologias usadas para medir o uso de álcool e a gravação dos registros de EEG durante o estado de sono, podem ser encontrados em Stoffer et al (1988).

A figura B.8 mostra o EEG categorizado durante o estado de sono (120 minutos) de um bebê aproximadamente 24-36 horas depois de nascimento, cuja mãe não consumiu bebidas alcoólicas durante sua gravidez. O correspondende EEG durante o estado de sono de um bebê cuja mãe consumiu bebidas alcoólicas durante sua gravidez é mostrado na figura B.9. Aqui, o estado de sono é categorizado (por minuto) em um dos seis possíveis estados: estado 1: sono profundo - sinal alternado; estado 2: sono profundo - alta voltagem; estado 3: sono indeterminado; estado 4: sono ativo - baixa voltagem; estado 5: sono ativo - misto; estado 6: acordado.

Denotamos por unexposed os dados mostrados na figura B.8 e exposed os dados mostrados na figura B.9. As transformadas de ondaletas de unexposed e exposed estão presentadas nas figuras B.10 e B.11, respectivamente. Consequentemente, as figuras B.12 e B.13 mostram seus periodogramas de ondaletas. Aqui, nessas figuras, "Resolution Level" corresponde ao nível $(M-j)$; nesse caso, $M=7$. Imagens desses periodogramas são representadas nas figuras B.18 e B.19. Esses periodogramas nos dizem como a energia numa série temporal é decomposta através das escalas, em tempos diferentes. As figuras B.18 e B.19 nos mostram, por exemplo, que o periodograma nas escalas mais altas contribuem mais para a energia em nossa série temporal que escalas mais baixas. Além disso, os menores valores dos periodogramas são apresentados na escala 1, os quais indicam a presença de ruído em série temporal. Por outro lado, comparando os dois periodogramas, note que existem valores maiores do periodograma nas escalas mais altas na figura B.19 que na figura B.18, e a distribuição da energia para cada nível $j$ na figura B.19 é mais uniforme que na figura B.18. Além disso, em níveis mais altos, a energia na figura B.18 é concentrada em valores pequenos de $k$, ao contrário que acontece na figura B.19, a qual é quase uniforme para cada $k$.

Agora vejamos o que acontece com o estad'o de sono reconstruído quando 
aplicamos limiar aos coeficientes de ondaletas ilustrados nas figuras B.10 e B.11, para remover os ruídos presentes em nossa série temporal. Aqui, usamos o valor de truncamento "universal". Seus periodogramas de ondaletas truncados estão mostrados nas figuras B.14 e B.15. Aqui notamos que os coeficientes dos periodogramas na escala 1 foram removidos, que é consistente com a análise apresentada acima. A figura B.16 mostra o estado de sono reconstruido de unexposed exposed.

Os escalogramas são apresentados na figura B.17. Notamos que existe um pico num nível alto $(j=5)$ nessas duas figuras. indicando que um periodo longo está presente nessas duas séries. No entanto, o pico apresentado em unexposed é muito mais forte que em exposed, indicando que a energia dessa série é concentrada em nível 5 e valores pequenos de $k$, de acordo com a análise acima. Também notamos os diferentes comportamentos dos escalogramas em escalas altas e baixas. A análise de Walsh-Fourier desses dados é dada em Stoffer et al (1988). Para detectar se álcool afeta o sono dos bebês ou não, sugerimos usar uma análise de variância usando ondaletas para comparar os dois grupos de bebês. 


\section{Capítulo 5}

\section{Estimação de Sistemas Lineares Variando no Tempo Utilizando Ondaletas}

\subsection{Introdução}

$\mathrm{Na}$ teoria dos processos estacionários, os sistemas lineares invariantes no tempo são de grande interesse. Se $X_{t}$ e $Y_{t}, t \in\{0, \pm 1, \cdots\}$, são dois processos estacionários, com média zero, então para as observações $\left\{X_{t}, Y_{t}, 1 \leq t \leq N\right\}$ queremos estimar os pesos do filtro $w_{u}$ no modelo

$$
Y_{t}=\sum_{u=-\infty}^{\infty} w_{u} X_{t-u}+\epsilon_{t},
$$

onde $\epsilon_{t}$ é um processo estacionário com média zero, ortogonal a $X_{t}$. Em particular, a soma infinita em (5.1) é substituida por uma finita. Para detalhes, ver Robinson (1979).

A estimação de $w_{u}$ envolve o espectro $f_{x x}(\lambda)$ de $X_{t}$, o espectro cruzado entre $X_{t}$ e $Y_{t}, f_{x y}(\lambda)$ e é implementada estimando em primeiro lugar a função de transferência $W(\lambda)=\sum_{u} w_{u} \exp (-i \lambda u)$. A estimação do espectro dos erros $f_{\epsilon \epsilon}(\lambda)$ também é de interesse. Ver Brillinger (1975, capítulo 8), para detalhes. 
Neste capítulo, consideramos os sistemas lineares variando no tempo, ou seja, onde os coeficientes do filtro são funções do tempo, isto é, $w_{u}(t)$. Para esta situação, os processos aparecendo em (5.1) vão ser considerados como localmente estacionários, no sentido de Dahlhaus (1997), definidos em (3.31) no Capítulo 3. Aqui, usaremos a expansão de ondaletas dos coeficientes variando no tempo para nossa aproximação.

A representação (3.31) é baseada numa sequência de funções $A_{t, T}^{o}(\lambda)$. Para facilitar a notação, usaremos neste capítulo a função $A(u, \lambda)$ no lugar de $A_{t, T}^{o}(\lambda)$, tal que a diferença $\left|A_{t, T}^{o}(\lambda)-A\left(\frac{t}{T}, \lambda\right)\right|$ é de $O\left(T^{-1}\right)$ e todos os resultados vão continuar valendo.

\subsection{Bases de Ondaletas Ortonormais e Esti- madores de Ondaletas do Espectro De- pendente do Tempo}

Vimos no Capítulo 2 que $\left\{\phi_{j, k} ; k \in Z\right\}$ é uma base ortonormal para $V_{j}$, $j \in Z$, o subespaço de $L^{2}(\mathcal{R})$ e $\left\{\psi_{j, k} ; k \in Z\right\}$ é uma base ortonormal para $W_{j}$, o complemento ortogonal de $V_{j}$ em $V_{j-1} ; \operatorname{logo},\left\{\psi_{j, k} ; j, k \in Z\right\}$ também é uma base ortonormal para $L^{2}(\mathcal{R})$.

Suponhamos que $\left\{\phi_{j, k} ; k \in Z\right\}$ geram $V_{j}$, o subespaço de $L^{2}[0,1]$ e $\left\{\tilde{\phi}_{j, k} ; k \in\right.$ $Z\}$ geram $\tilde{V}_{j}$, o subespaço de $L^{2}[-\pi, \pi]$, então

$$
L^{2}([0,1] \times[-\pi, \pi])=\bigcup_{j=l}^{\infty} V_{j} \otimes \tilde{V}_{j}
$$

que mostra a possibilidade de construir uma base $\mathcal{B}=\left\{\mathcal{U}_{I}(u, \lambda)\right\}$ de $L^{2}([0,1] \times$ $[-\pi, \pi])$ como sendo o produto de duas bases unidimensionais $\left\{\phi_{l, k} ; k \in Z\right\} \cup$ $\left\{\psi_{j, k} ; j \geq l, k \in Z\right\}$ (na direção de tempo) e $\left\{\tilde{\phi}_{l, k} ; k \in Z\right\} \cup\left\{\tilde{\psi}_{j, k} ; j \geq l, k \in Z\right\}$ (na direção de frequência), como proposto em Daubechies (1992).

Para facilitar a notação escrevemos $\psi_{l-1, k}$ e $\tilde{\psi}_{l-1, k}$ no lugar de $\phi_{l, k}$ e $\tilde{\phi}_{l, k}$, respectivamente e usaremos $\mathcal{I}=\left(j_{1}, j_{2}, k_{1}, k_{2}\right)$.

Agora, suponhamos que temos uma sequência de observações $\left\{X_{1, T}, \cdots, X_{T, T}\right\}$ e baseadas nessas observações queremos estimar o espectro dependente do tempo $f(u, \lambda)$, definido por (3.32). 
Neumann e von Sachs (1997) introduziram uma estatística chamada periodograma, $I_{t, T}, 1 \leq t \leq T$,

$$
I_{t, T}(\lambda)=\frac{1}{2 \pi} \sum_{|s| \leq \min \{t-1, T-t\}} X_{[t-s / 2], T} X_{[t+s / 2], T} \exp (-i \lambda s),
$$

que pode ser considerada como um estimador preliminar de $f(u, \lambda)$.

Os verdadeiros coeficientes de ondaleta $\Theta_{\mathcal{I}}$ de $f(u, \lambda)$ são definidos como

$$
\Theta_{\mathcal{I}}=\int_{U \times \Pi} f(u, \lambda) \mathcal{U}_{\mathcal{I}}(u, \lambda) d u d \lambda=\int_{U \times \Pi} f(u, \lambda) \psi_{j_{1}, k_{1}}(u) \tilde{\psi}_{j_{2}, k_{2}}(\lambda) d u d \lambda
$$

onde $U \times \Pi=[0,1] \times[-\pi, \pi]$.

Portanto, temos

$$
f(u, \lambda)=\sum_{\mathcal{I}} \Theta_{\mathcal{I}} \psi_{j_{1}, k_{1}}(u) \tilde{\psi}_{j_{2}, k_{2}}(\lambda)
$$

Agora definimos os coeficientes empíricos de ondaleta como

$$
\hat{\Theta}_{\mathcal{I}}=\sum_{t=1}^{T} \int_{\frac{t-1}{T}}^{\frac{t}{T}} \psi_{j_{1}, k_{1}}(u) d u \int_{-\pi}^{\pi} \tilde{\psi}_{j_{2}, k_{2}}(\lambda) I_{t, T}(\lambda) d \lambda
$$

de modo que um estimador de $f(u, \lambda)$ pode ser tomado como

$$
\hat{f}(u, \lambda)=\sum_{\mathcal{I} \in \mathcal{I}_{T}} \hat{\Theta}_{\mathcal{I}} \psi_{j_{1}, k_{1}}(u) \tilde{\psi}_{j_{2}, k_{2}}(\lambda),
$$

$\operatorname{com} \mathcal{I}_{T}=\left\{\mathcal{I}: 2^{j_{1}+j_{2}} \leq T^{1-\delta}\right\}$, para alguma $0<\delta<1$.

Para obter as propriedades assintóticas dos coeficientes $\hat{\Theta}_{\mathcal{I}}$, precisamos algumas suposições.

Definição 5.1: A variação total em $U \times \Pi=[0,1] \times[-\pi, \pi]$ é dada por

$T V_{U \times \Pi}(f)=\sup \sum_{i} \sum_{j}\left|f\left(u_{i}, \lambda_{j}\right)-f\left(u_{i}, \lambda_{j-1}\right)-f\left(u_{i-1}, \lambda_{j}\right)+f\left(u_{i-1}, \lambda_{j-1}\right)\right|$,

onde o supremo é tomado sobre todas as partições de $U \times \Pi$.

\section{Suposições:}

(S1) $A(u, \lambda)$ tem variação total limitada em $U \times \Pi$; 
(S2) $\sup _{u} T V_{[-\pi, \pi]}(A(u, \cdot))<\infty \operatorname{esup}_{\lambda} T V_{[0,1]}(A(\cdot, \lambda))<\infty$;

(S3) $\sup _{u, \lambda}|A(u, \lambda)|<\infty$;

(S4) $\inf _{u, \lambda}|A(u, \lambda)| \geq k$ para algum $k>0$;

(S5) seja $\hat{A}(u, s)=\frac{1}{2 \pi} \int A(u, \lambda) \exp (i \lambda s) d \lambda, s \in \mathcal{Z}, u \in[0,1]$, então

$$
\sup _{u} \sum_{s}|\hat{A}(u, s)|<\infty
$$

(S6) $\phi(u), \psi(u), \tilde{\phi}(\lambda), \tilde{\psi}(\lambda)$ têm variação total limitada em $[0,1]$ e $[-\pi, \pi]$, respectivamente;

(S7) $\sum_{s}|\hat{\tilde{\phi}}(s)|<\infty$ e $\sum_{\tilde{\phi}}|\hat{\tilde{\psi}}(s)|<\infty$, onde $\hat{\tilde{\phi}}(s)$ e $\hat{\tilde{\psi}}(s)$ são as transformadas de Fourier de $\tilde{\phi}(s)$ e $\tilde{\psi}(s)$, respectivamente.

(S8)

$$
\begin{gathered}
\sup _{1 \leq t_{1} \leq T}\left\{\sum_{t_{2}, \cdots, t_{k}=1}^{T}\left|\operatorname{Cum}\left(X_{t_{1}, T}, \cdots, X_{t_{k}, T}\right)\right|\right\} \leq C^{k}(k !)^{1+\gamma}, \\
\sup _{1 \leq t_{1} \leq T}\left\{\sum_{t_{2}, \cdots, t_{k}=1}^{T}\left|\operatorname{Cum}\left(Y_{t_{1}, T}, \cdots, Y_{t_{k}, T}\right)\right|\right\} \leq C^{k}(k !)^{1+\gamma},
\end{gathered}
$$

para todo $k=2,3, \cdots$, onde $\gamma \geq 0$.

(S9) $f_{x x}(u, \lambda) \neq 0$ e $\hat{f}_{x x}(u, \lambda) \neq 0, \forall u, \lambda$.

Neumann and von Sachs (1997) (Lema 3.2) obtiveram os seguintes resultados para $\hat{\Theta}_{\mathcal{I}}$ :

Lema 5.1: Suponha válidas (S1) a (S8), para todo $\hat{\Theta}_{\mathcal{I}} \operatorname{com} 2^{j_{1}+j_{2}}=o(T)$ e $j_{2}^{-1}=o(1)$. Então,

(a)

$$
E\left\{\hat{\Theta}_{\mathcal{I}}\right\}=\Theta_{\mathcal{I}}+o\left(T^{-1 / 2}\right)
$$

(b)

$$
\begin{aligned}
\operatorname{Var}\left\{\hat{\Theta}_{\mathcal{I}}\right\} & =2 \pi T^{-1} \int_{U \times \Pi}\left\{f(u, \lambda) \psi_{j_{1}, k_{1}}(u)\right\}^{2} d u \tilde{\psi}_{j_{2}, k_{2}}(\lambda)\left[\tilde{\psi}_{j_{2}, k_{2}}(\lambda)\right. \\
& \left.+\tilde{\psi}_{j_{2}, k_{2}}(-\lambda)\right] d \lambda+o\left(T^{-1}\right)+O\left(2^{-j_{2}} T^{-1}\right)
\end{aligned}
$$


(c)

$$
\left|\operatorname{Cum}_{k}\left(\hat{\Theta}_{\mathcal{I}}\right)\right| \leq(k !)^{2+2 \gamma} C^{k} T^{-1}\left(T^{-1} 2^{\left(j_{1}+j_{2}\right) / 2} \log (T)\right)^{k-2}
$$

para $k \geq 3$ e $C>0$ apropriado, uniformemente em $\mathcal{I} \in \mathcal{I}_{T}$.

Assim, de (a) e (b) temos que $\hat{\Theta}_{\mathcal{I}}$ é assintoticamente não viciado e consistente. De (c) temos a normalidade assintótica.

\subsection{Sistemas Lineares Variando no Tempo}

Consideremos um sistema linear

$$
Y_{t, T}=\sum_{u} a_{u}\left(\frac{t}{T}\right) X_{t-u, T}+\epsilon_{t}, \quad t=1, \cdots, T,
$$

onde:

(i) $Y_{t, T}, X_{t, T}$ são processos localmente estacionários com média zero;

(ii)

$$
\sup _{v} \sum_{u}\left|a_{u}(v)\right|<\infty
$$

(iii) $\epsilon_{t}$ é uma série estacionária, média zero, ortogonal a $X_{t, T}$.

Consideremos a expansão

$$
a_{u}\left(\frac{t}{T}\right)=\sum_{j} \sum_{k} \beta_{j, k}^{(u)} \psi_{j, k}\left(\frac{t}{T}\right), \quad j, k \in Z
$$

onde

$$
\beta_{j, k}^{(u)}=\int_{0}^{1} a_{u}(v) \psi_{j, k}(v) d v
$$

A seguir, pretendemos achar um estimador $\hat{\beta}_{j, k}^{(u)}$ para estimar $\beta_{j, k}^{(u)}$ e obter algumas propriedades assintóticas de $\hat{\beta}_{j, k}^{(u)}$. Consequentemente, as propriedades assintóticas dos estimadores de $a_{u}\left(\frac{t}{T}\right)$ seguir-se-ão.

Em primeiro lugar, para o processo $X_{t, T}$, definimos

$$
C_{x x}(u, k)=\frac{1}{2 \pi} \int_{-\pi}^{\pi} f_{x x}(u, \lambda) \exp (i \lambda k) d \lambda,
$$


como a covariância local de lag $k$ no tempo $u$, onde $f_{x x}(u, \lambda)$ é o espectro dependente do tempo de $X_{t, T}$. Então temos (Dahlhaus, 1996):

$$
\begin{aligned}
C_{x x}^{(T)}\left(\frac{t}{T}, k\right) & =\dot{C} \operatorname{Cov}\left\{X_{[t-k / 2], T}, X_{[t+k / 2], T}\right\} \\
& =\int_{-\pi}^{\pi} A\left(\frac{t-k / 2}{T}, \lambda\right) A\left(\frac{t+k / 2}{T}, \lambda\right) \exp (i \lambda k) d \lambda \\
& =C_{x x}\left(\frac{t}{T}, k\right)+C^{\prime}\left(\frac{t}{T}, k\right) O\left(\frac{k}{T}\right)
\end{aligned}
$$

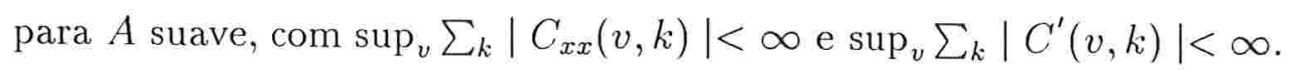

Analogamente, para os processos $X_{t, T}$ e $Y_{t, T}$, definamos

$$
C_{x y}(u, k)=\frac{1}{2 \pi} \int_{-\pi}^{\pi} f_{x y}(u, \lambda) \exp (i \lambda k) d \lambda
$$

como a covariância cruzada local de lag $k$ no tempo $u$, onde $f_{x y}(u, \lambda)$ é o espectro cruzado dependente do tempo de $X_{t, T}$ e $Y_{t, T}$, definido por

$$
f_{x y}(u, \lambda)=\lim _{T \rightarrow \infty} \frac{1}{2 \pi} \sum_{s=-\infty}^{\infty} \operatorname{Cov}\left\{X_{[u T-s / 2], T}, Y_{[u T+s / 2], T}\right\} \exp (-i \lambda s) .
$$

Agora, temos, para cada $m \in \mathcal{Z}$,

$$
\begin{aligned}
\operatorname{Cov}\left\{Y_{t, T}, X_{t-m, T}\right\} & =E\left\{Y_{t, T} X_{t-m, T}\right\} \\
& =E\left\{\left[\sum_{u} a_{u}\left(\frac{t}{T}\right) X_{t-u, T}+\epsilon_{t}\right] X_{t-m, T}\right\} \\
& =E\left\{\sum_{u} a_{u}\left(\frac{t}{T}\right) X_{t-u, T} X_{t-m, T}\right\}+E\left\{\epsilon_{t} X_{t-m, T}\right\} \\
& =\sum_{u} a_{u}\left(\frac{t}{T}\right) E\left\{X_{t-u, T} X_{t-m, T}\right\} \\
& =\sum_{u} a_{u}\left(\frac{t}{T}\right) \operatorname{Cov}\left\{X_{t-u, T}, X_{t-m, T}\right\} \\
& =\sum_{u} a_{u}\left(\frac{t}{T}\right)\left\{C_{x x}\left(\frac{t}{T},(m-u)\right)+O\left(T^{-1}\right)\right\} \\
& =\sum_{u} a_{u}\left(\frac{t}{T}\right) \frac{1}{2 \pi} \int_{-\pi}^{\pi} f_{x x}\left(\frac{t}{T}, \lambda\right) \exp (i \lambda(m-u)) d \lambda+O\left(T^{-1}\right) \\
& =\frac{1}{2 \pi} \int_{-\pi}^{\pi} \sum_{u} a_{u}\left(\frac{t}{T}\right) \exp (-i \lambda u) f_{x x}\left(\frac{t}{T}, \lambda\right) \exp (i \lambda m) d \lambda+O\left(T^{-1}\right)
\end{aligned}
$$




$$
=\frac{1}{2 \pi} \int_{-\pi}^{\pi} B_{a}\left(\frac{t}{T}, \lambda\right) f_{x x}\left(\frac{t}{T}, \lambda\right) \exp (i \lambda m) d \lambda+O\left(T^{-1}\right),
$$

onde

$$
B_{a}\left(\frac{t}{T}, \lambda\right)=\sum_{u} a_{u}\left(\frac{t}{T}\right) \exp (-i \lambda u)
$$

Então,

$$
f_{x y}\left(\frac{t}{T}, \lambda\right)=B_{a}\left(\frac{t}{T}, \lambda\right) f_{x x}\left(\frac{t}{T}, \lambda\right)+O\left(T^{-1}\right) .
$$

Portanto $B_{a}\left(\frac{t}{T}, \lambda\right)$ pode ser estimado por

$$
\hat{B}_{a}\left(\frac{t}{T}, \lambda\right)=\hat{f}_{x y}\left(\frac{t}{T}, \lambda\right)\left\{\hat{f}_{x x}\left(\frac{t}{T}, \lambda\right)\right\}^{-1},
$$

onde

$$
\begin{gathered}
\hat{f}_{x x}\left(\frac{t}{T}, \lambda\right)=\sum_{\mathcal{I} \in \mathcal{I}_{T}} \hat{\Theta}_{\mathcal{I}}^{(x x)} \psi_{j_{1}, k_{1}}\left(\frac{t}{T}\right) \tilde{\psi}_{j_{2}, k_{2}}(\lambda), \\
\hat{\Theta}_{\mathcal{I}}^{(x x)}=\sum_{t=1}^{T} \int_{\frac{t-1}{T}}^{\frac{t}{T}} \psi_{j_{1}, k_{1}}(u) d u \int_{-\pi}^{\pi} \tilde{\psi}_{j_{2}, k_{2}}(\lambda) I_{t, T}^{(x x)}(\lambda) d \lambda, \\
I_{t, T}^{(x x)}(\lambda)=\frac{1}{2 \pi} \sum_{|s| \leq m i n\{t-1, T-t\}} X_{[t-s / 2], T} X_{[t+s / 2], T} \exp (-i \lambda s), \\
\mathcal{I}_{T}=\left\{\mathcal{I}: 2^{j_{1}+j_{2}} \leq T^{1-\delta}\right\}, 0<\delta<1, \quad \mathcal{I}=\left\{j_{1}, j_{2}, k_{1}, k_{2}\right\} .
\end{gathered}
$$

Da mesma maneira,

$$
\begin{gathered}
\hat{f}_{x y}\left(\frac{t}{T}, \lambda\right)=\sum_{\mathcal{I} \in \mathcal{I}_{T}} \hat{\Theta}_{\mathcal{I}}^{(x y)} \psi_{j_{1}, k_{1}}\left(\frac{t}{T}\right) \tilde{\psi}_{j_{2}, k_{2}}(\lambda), \\
\hat{\Theta}_{\mathcal{I}}^{(x y)}=\sum_{t=1}^{T} \int_{\frac{t-1}{T}}^{\frac{t}{T}} \psi_{j_{1}, k_{1}}(u) d u \int_{-\pi}^{\pi} \tilde{\psi}_{j_{2}, k_{2}}(\lambda) I_{t, T}^{(x y)}(\lambda) d \lambda,
\end{gathered}
$$


$\mathrm{e}$

$$
I_{t, T}^{(x y)}(\lambda)=\frac{1}{2 \pi} \sum_{|s| \leq \min \{t-1, T-t\}} X_{[t-s / 2], T} Y_{[t+s / 2], T} \exp (-i \lambda s)
$$

De (5.19) temos

$$
a_{u}\left(\frac{t}{T}\right)=\frac{1}{2 \pi} \int_{-\pi}^{\pi} B_{a}\left(\frac{t}{T}, \lambda\right) \exp (i \lambda u) d \lambda
$$

para $u=0, \pm 1, \cdots$. Portanto, $a_{u}\left(\frac{t}{T}\right)$ pode ser estimado por

$$
\hat{a}_{u}\left(\frac{t}{T}\right)=P_{T}^{-1} \sum_{p=0}^{P_{T}-1} \hat{B}_{a}\left(\frac{t}{T}, \frac{p}{P_{T}}\right) \exp \left(i u \frac{p}{P_{T}}\right)
$$

onde $P_{T}$ é uma sequência de inteiros tendendo para $\infty$ quando $T \rightarrow \infty$ e $\hat{a}_{u}\left(\frac{t}{T}\right)$ é considerado como um estimador preliminar de $a_{u}\left(\frac{t}{T}\right)$.

Finalmente, a partir de (5.14), podemos estimar $\beta_{j, k}^{(u)}$ através de

$$
\begin{aligned}
\hat{\beta}_{j, k}^{(u)}= & \frac{1}{T} \sum_{t=0}^{T-1} \hat{a}_{u}\left(\frac{t}{T}\right) \psi_{j, k}\left(\frac{t}{T}\right) \\
= & \frac{1}{T} \sum_{t=0}^{T-1} \frac{1}{P_{T}} \sum_{p=0}^{P_{T}-1} \hat{B}_{a}\left(\frac{t}{T}, \frac{p}{P_{T}}\right) \exp \left(i u \frac{p}{P_{T}}\right) \psi_{j, k}\left(\frac{t}{T}\right) \\
= & \frac{1}{T} \sum_{t=0}^{T-1} \frac{1}{P_{T}} \sum_{p=0}^{P_{T}-1} \hat{f}_{x y}\left(\frac{t}{T}, \frac{p}{P_{T}}\right)\left\{\hat{f}_{x x}\left(\frac{t}{T}, \frac{p}{P_{T}}\right)\right\}^{-1} \\
& \exp \left(i u \frac{p}{P_{T}}\right) \psi_{j, k}\left(\frac{t}{T}\right) .
\end{aligned}
$$

De (5.13), segue-se que estimamos $a_{u}\left(\frac{t}{T}\right)$ por

$$
\tilde{a}_{u}\left(\frac{s}{T}\right)=\sum_{j=0}^{J_{T}} \sum_{k} \hat{\beta}_{j, k}^{(u)} \psi_{j, k}\left(\frac{s}{T}\right),
$$

para algum valor grande de $J_{T}$. Como $\psi$ tem suporte compacto, o número de $k$ para o qual $\psi_{j, k}(\cdot) \neq 0$ é limitado, assim somente um número finito de termos são envolvidos em (5.24). 
Usando o lema 5.1, obtemos os seguintes resultados para $\hat{\beta}_{j, k}^{(u)}$ e $\tilde{a}_{u}\left(\frac{s}{T}\right)$.

Teorema 5.1: Assumindo as suposições (S1) a (S9), temos

(a)

$$
E\left\{\hat{\beta}_{j, k}^{(u)}\right\}=\beta_{j, k}^{(u)}+O\left(T^{-1}\right)
$$

(b)

$$
\begin{aligned}
& \operatorname{Cov}\left\{\hat{\beta}_{j, k}^{(u)}, \hat{\beta}_{j^{\prime}, k^{\prime}}^{\left(u^{\prime}\right)}\right\}= \\
& \frac{1}{T^{2}} \sum_{t, t^{\prime}=0}^{T-1} \frac{1}{P_{T}^{2}} \sum_{p, p^{\prime}=0}^{P_{T}-1} \frac{\sum_{\mathcal{I}}\left[C_{\mathcal{I}}^{(x y)}+C_{\mathcal{I}}^{(x x)} B_{a}\left(\frac{t}{T}, \frac{p}{P_{T}}\right) B_{a}\left(\frac{t^{\prime}}{T}, \frac{p^{\prime}}{P_{T}}\right)\right] \Psi_{\mathcal{I}}}{f_{x x}\left(\frac{t}{T}, \frac{p}{P_{T}}\right) f_{x x}\left(\frac{t^{\prime}}{T}, \frac{p^{\prime}}{P_{T}}\right)} \\
& \quad \exp \left[i\left(u \frac{p}{P_{T}}+u^{\prime} \frac{p^{\prime}}{P_{T}}\right)\right] \psi_{j, k}\left(\frac{t}{T}\right) \psi_{j^{\prime}, k^{\prime}}\left(\frac{t^{\prime}}{T}\right)+O\left(T^{-2-\delta}\right) ;
\end{aligned}
$$

para algum $\delta>0 . \operatorname{Em}(5.26)$,

$$
\begin{gathered}
C_{\mathcal{I}}^{(x x)}=\frac{2 \pi}{T} \int_{U \times \Pi}\left\{f_{x x}(u, \lambda) \psi_{j_{1}, k_{1}}(u)\right\}^{2} \tilde{\psi}_{j_{2}, k_{2}}(\lambda)\left[\tilde{\psi}_{j_{2}, k_{2}}(\lambda)+\tilde{\psi}_{j_{2}, k_{2}}(-\lambda)\right] d u d \lambda, \\
C_{\mathcal{I}}^{(x y)}=\frac{2 \pi}{T} \int_{U \times \Pi}\left\{f_{x y}(u, \lambda) \psi_{j_{1}, k_{1}}(u)\right\}^{2} \tilde{\psi}_{j_{2}, k_{2}}(\lambda)\left[\tilde{\psi}_{j_{2}, k_{2}}(\lambda)+\tilde{\psi}_{j_{2}, k_{2}}(-\lambda)\right] d u d \lambda, \\
\Psi_{\mathcal{I}}=\psi_{j_{1}, k_{1}}\left(\frac{t}{T}\right) \psi_{j_{1}, k_{1}}\left(\frac{t^{\prime}}{T}\right) \tilde{\psi}_{j_{2}, k_{2}}\left(\frac{p}{P_{T}}\right) \tilde{\psi}_{j_{2}, k_{2}}\left(\frac{p^{\prime}}{P_{T}}\right),
\end{gathered}
$$

e temos $\sum_{\mathcal{I}} C_{\mathcal{I}}^{(x x)} \Psi_{\mathcal{I}}=O\left(T^{-\delta}\right)$ e $\sum_{\mathcal{I}} C_{\mathcal{I}}^{(x y)} \Psi_{\mathcal{I}}=O\left(T^{-\delta}\right)$; os erros em (5.25) e (5.26) são uniformes em $\mathcal{I}$.

(c) $\hat{\beta}_{j, k}^{(u)}$ tem uma distribuição assintoticamente normal, com média $\beta_{j, k}^{(u)}$ e a estrutura de covariância dada por (5.26).

Para demonstrar o teorema 5.1, precisamos dos dois lemas a seguir.

Lema 5.2: (Brillinger, 1975, Teorema P5.2)

Seja uma sequência de vetores aleatórios r-dimensionais, $\sqrt{T}\left(Y_{T}-\mu\right)$, $\mu=\left(\mu_{1}, \mu_{2}, \cdots, \mu_{r}\right), T=1,2, \cdots$, tendendo em distribuição para $N_{r}(0, \Sigma)$. 
Seja $g: R^{r} \rightarrow R^{s}$ uma função s-dimensional diferenciável numa vizinhança de $\mu$ e seja $J$ a matriz Jacobiana $J$ em $\mu$. Então,

$$
\sqrt{T}\left\{g\left(Y_{T}\right)-g(\mu)\right\} \stackrel{\mathcal{D}}{\rightarrow} N_{s}\left(0, J \Sigma J^{\prime}\right)
$$

quando $T \rightarrow \infty$.

Lema 5.3: (Mardia, Kent e Bibby, 1979, Teorema 3.1.1)

Se $X$ tem uma distribuição normal $p$-variada, e se $Y=A X+c$, onde $A$ é qualquer matrix $(q \times p)$ e $c$ qualquer vetor com dimensão $p$, então $Y$ tem uma distribuição normal $q$-variada.

\section{Demonstração:}

(a) A partir de (5.20), temos

$$
\hat{B}_{a}\left(\frac{t}{T}, \lambda\right)=\hat{f}_{x y}\left(\frac{t}{T}, \lambda\right)\left\{\hat{f}_{x x}\left(\frac{t}{T}, \lambda\right)\right\}^{-1}, \quad \hat{f}_{x x}\left(\frac{t}{T}, \lambda\right) \neq 0 .
$$

Agora, usando (5.7) e (5.8),

$$
\begin{aligned}
E\left\{\hat{f}_{x x}\left(\frac{t}{T}, \lambda\right)\right\} & =E\left\{\sum_{\mathcal{I} \in \mathcal{I}_{T}} \hat{\Theta}_{\mathcal{I}}^{(x x)} \psi_{j_{1}, k_{1}}\left(\frac{t}{T}\right) \tilde{\psi}_{j_{2}, k_{2}}(\lambda)\right\} \\
& =\sum_{\mathcal{I} \in \mathcal{I}_{T}} E\left\{\hat{\Theta}_{\mathcal{I}}^{(x x)}\right\} \psi_{j_{1}, k_{1}}\left(\frac{t}{T}\right) \tilde{\psi}_{j_{2}, k_{2}}(\lambda) \\
& =\sum_{\mathcal{I} \in \mathcal{I}_{T}}\left\{\Theta_{\mathcal{I}}^{(x x)}+o\left(T^{-1 / 2}\right)\right\} \psi_{j_{1}, k_{1}}\left(\frac{t}{T}\right) \tilde{\psi}_{j_{2}, k_{2}}(\lambda) \\
& =f_{x x}\left(\frac{t}{T}, \lambda\right)+R_{1},
\end{aligned}
$$

onde

$$
\begin{aligned}
\left|R_{1}\right| & \leq o\left(T^{-1 / 2}\right) \sum_{\mathcal{I}}\left|\psi_{j_{1}, k_{1}}\left(\frac{t}{T}\right) \| \tilde{\psi}_{j_{2}, k_{2}}(\lambda)\right| \\
& \leq o\left(T^{-1 / 2}\right) \sum_{j_{1}=1}^{J_{1}} \sum_{j_{2}=1}^{J_{2}} 2^{j_{1} / 2} A_{1} 2^{j_{2} / 2} A_{2} \\
& \leq o\left(T^{-1 / 2}\right) \sum_{j_{1}, j_{2}} 2^{\left(j_{1}+j_{2}\right) / 2} A_{1} A_{2} .
\end{aligned}
$$

Portanto, $R_{1}=o\left(T^{-1 / 2}\right) O\left(2^{\left(J_{1}+J_{2}\right) / 2}\right)=o\left(T^{-1 / 2} T^{(1-\delta) / 2}\right)=o\left(T^{-\delta / 2}\right)$ com $|\psi| \leq A_{1}$ e $|\tilde{\psi}| \leq A_{2}$. 
Daí,

$$
E\left\{\hat{f}_{x x}\left(\frac{t}{T}, \lambda\right)\right\}=f_{x x}\left(\frac{t}{T}, \lambda\right)+o\left(T^{-\delta / 2}\right), \quad \text { para } \delta>0 .
$$

Observação: Em vários lugares o fato de que $\psi(x)$ tem suporte compacto, para um dado $x$, o número de $k$ para o qual $\psi_{j, k}(x) \neq 0$ é limitado, uniformemente em $j$, por $2^{j / 2} \mid$ support $\psi \mid$, foi usado.

Analogamente, temos

$$
E\left\{\hat{f}_{x y}\left(\frac{t}{T}, \lambda\right)\right\}=f_{x y}\left(\frac{t}{T}, \lambda\right)+o\left(T^{-\delta / 2}\right), \quad \text { para } \delta>0 .
$$

Pelo Teorema 5.4.3 de Fuller (1996),

$$
\begin{aligned}
E\left\{\hat{B}_{a}\left(\frac{t}{T}, \lambda\right)\right\} & =E\left\{\hat{f}_{x y}\left(\frac{t}{T}, \lambda\right)\left[\hat{f}_{x x}\left(\frac{t}{T}, \lambda\right)\right]^{-1}\right\} \\
& =\frac{E\left\{\hat{f}_{x y}\left(\frac{t}{T}, \lambda\right)\right\}}{E\left\{\hat{f}_{x x}\left(\frac{t}{T}, \lambda\right)\right\}}+O\left(a_{n}^{2}\right) \\
& =\frac{f_{x y}\left(\frac{t}{T}, \lambda\right)+o\left(T^{-\delta / 2}\right)}{f_{x x}\left(\frac{t}{T}, \lambda\right)+o\left(T^{-\delta / 2}\right)}+O\left(T^{-\delta}\right) \\
& =\frac{B_{a}\left(\frac{t}{T}, \lambda\right) f_{x x}\left(\frac{t}{T}, \lambda\right)+O\left(T^{-1}\right)+o\left(T^{-\delta / 2}\right)}{f_{x x}\left(\frac{t}{T}, \lambda\right)+o\left(T^{-\delta / 2}\right)}+O\left(T^{-\delta}\right),
\end{aligned}
$$

onde

$$
a_{n}=E\left|\begin{array}{c}
\hat{f}_{x y}\left(\frac{t}{T}, \lambda\right)-E\left[\hat{f}_{x y}\left(\frac{t}{T}, \lambda\right)\right] \\
\hat{f}_{x x}\left(\frac{t}{T}, \lambda\right)-E\left[\hat{f}_{x x}\left(\frac{t}{T}, \lambda\right)\right]
\end{array}\right|^{2}
$$

e $O\left(a_{n}\right)=O\left(T^{-\delta}\right)$.

Assim a partir de (5.23), temos

$$
\begin{aligned}
E\left\{\hat{\beta}_{j, k}^{(u)}\right\}= & \frac{1}{T} \frac{1}{P_{T}} \sum_{t=0}^{T-1} \sum_{p=0}^{P_{T}-1} E\left\{\hat{B}_{a}\left(\frac{t}{T}, \frac{p}{P_{T}}\right)\right\} \exp \left(i u \frac{p}{P_{T}}\right) \psi_{j, k}\left(\frac{t}{T}\right) \\
= & \frac{1}{T} \frac{1}{P_{T}} \sum_{t=0}^{T-1} \sum_{p=0}^{P_{T}-1}\left\{\frac{B_{a}\left(\frac{t}{T}, \frac{p}{P_{T}}\right) f_{x x}\left(\frac{t}{T}, \frac{p}{P_{T}}\right)+O\left(T^{-1}\right)+o\left(T^{-\delta / 2}\right)}{f_{x x}\left(\frac{t}{T}, \frac{p}{P_{T}}\right)+o\left(T^{-\delta / 2}\right)}+O\left(T^{-\delta}\right)\right\} \\
& \cdot \exp \left(i u \frac{p}{P_{T}}\right) \psi_{j, k}\left(\frac{t}{T}\right)
\end{aligned}
$$




$$
\begin{aligned}
= & \frac{1}{T} \frac{1}{P_{T}} \sum_{t=0}^{T-1} \sum_{p=0}^{P_{T}-1} \frac{B_{a}\left(\frac{t}{T}, \frac{p}{P_{T}}\right) f_{x x}\left(\frac{t}{T}, \frac{p}{P_{T}}\right)+O\left(T^{-1}\right)+o\left(T^{-\delta / 2}\right)}{f_{x x}\left(\frac{t}{T}, \frac{p}{P_{T}}\right)+o\left(T^{-\delta / 2}\right)} \exp \left(i u \frac{p}{P_{T}}\right) \psi_{j, k}\left(\frac{t}{T}\right) \\
& +\frac{1}{T} \frac{1}{P_{T}} \sum_{t=0}^{T-1} \sum_{p=0}^{P_{T}-1} O\left(T^{-\delta}\right) \exp \left(i u \frac{p}{P_{T}}\right) \psi_{j, k}\left(\frac{t}{T}\right) \\
= & S_{1}+S_{2} .
\end{aligned}
$$

Agora,

$$
\begin{aligned}
\left|S_{2}\right| & =\left|\frac{1}{T} \frac{1}{P_{T}} \sum_{t=0}^{T-1} \sum_{p=0}^{P_{T}-1} O\left(T^{-\delta}\right) \exp \left(i u \frac{p}{P_{T}}\right) \psi_{j, k}\left(\frac{t}{T}\right)\right| \\
& \leq\left|\frac{1}{T} \sum_{t=0}^{T-1} \psi_{j, k}\left(\frac{t}{T}\right) \| \frac{1}{P_{T}} \sum_{p=0}^{P_{T}-1} \exp \left(i u \frac{p}{P_{T}}\right)\right| O\left(T^{-\delta}\right)
\end{aligned}
$$

Portanto, $S_{2}=O\left(T^{-1-\delta}\right)$.

Quando $T \rightarrow \infty$,

$$
\begin{aligned}
S_{1}= & \frac{1}{T} \sum_{t=0}^{T-1} \frac{1}{P_{T}} \sum_{p=0}^{P_{T}-1} B_{a}\left(\frac{t}{T}, \frac{p}{P_{T}}\right) \exp \left(i u \frac{p}{P_{T}}\right) \psi_{j, k}\left(\frac{t}{T}\right) \\
= & \frac{1}{T} \sum_{t=0}^{T-1} \psi_{j, k}\left(\frac{t}{T}\right) \frac{1}{P_{T}} \sum_{p=0}^{P_{T}-1}\left\{\sum_{u^{\prime}} a_{u^{\prime}}\left(\frac{t}{T}\right) \exp \left(-i u^{\prime} \frac{p}{P_{T}}\right)\right\} \exp \left(i u \frac{p}{P_{T}}\right) \\
= & \frac{1}{T} \sum_{t=0}^{T-1} \psi_{j, k}\left(\frac{t}{T}\right) \frac{1}{P_{T}} \sum_{p=0}^{P_{T}-1} a_{u}\left(\frac{t}{T}\right) \exp \left(-i u \frac{p}{P_{T}}\right) \exp \left(i u \frac{p}{P_{T}}\right) \\
& +\frac{1}{T} \sum_{t=0}^{T-1} \psi_{j, k}\left(\frac{t}{T}\right) \frac{1}{P_{T}} \sum_{p=0}^{P_{T}-1} \sum_{u^{\prime} \neq u} a_{u^{\prime}}\left(\frac{t}{T}\right) \exp \left(-i \frac{p}{P_{T}}\left(u^{\prime}-u\right)\right) \\
= & \frac{1}{T} \sum_{t=0}^{T-1} \psi_{j, k}\left(\frac{t}{T}\right) a_{u}\left(\frac{t}{T}\right)+S_{3} .
\end{aligned}
$$

Por um Lema de Polya e Szegö(1925), obtemos

$$
\left|\frac{1}{T} \sum_{t=0}^{T-1} \psi_{j, k}\left(\frac{t}{T}\right) a_{u}\left(\frac{t}{T}\right)-\int a_{u}\left(\frac{t}{T}\right) \psi_{j, k}\left(\frac{t}{T}\right) d t\right| \leq \frac{V}{T}
$$


onde $V$ é a variação de $a_{u}\left(\frac{t}{T}\right) \psi_{j, k}\left(\frac{t}{T}\right)$. Portanto,

$$
\begin{aligned}
\frac{1}{T} \sum_{t=0}^{T-1} \psi_{j, k}\left(\frac{t}{T}\right) a_{u}\left(\frac{t}{T}\right) & =\int a_{u}\left(\frac{t}{T}\right) \psi_{j, k}\left(\frac{t}{T}\right) d t+O\left(T^{-1}\right) \\
& =\beta_{j, k}^{(u)}+O\left(T^{-1}\right)
\end{aligned}
$$

e

$$
\begin{aligned}
\left|S_{3}\right| & =\left|\frac{1}{T} \sum_{t=0}^{T-1} \psi_{j, k}\left(\frac{t}{T}\right) \frac{1}{P_{T}} \sum_{p=0}^{P_{T}-1} \sum_{u^{\prime} \neq u} a_{u^{\prime}}\left(\frac{t}{T}\right) \exp \left(-i \frac{p}{P_{T}}\left(u^{\prime}-u\right)\right)\right| \\
& \leq\left|\frac{1}{T} \sum_{t=0}^{T-1} \psi_{j, k}\left(\frac{t}{T}\right)\right| \frac{1}{P_{T}} \sum_{p=0}^{P_{T}-1} \sum_{u^{\prime} \neq u}\left|a_{u^{\prime}}\left(\frac{t}{T}\right)\right|\left|\exp \left(-i \frac{p}{P_{T}}\left(u^{\prime}-u\right)\right)\right| .
\end{aligned}
$$

Portanto, $S_{3}=O\left(T^{-1}\right)$.

Daí, obtemos

$$
E\left\{\hat{\beta}_{j, k}^{(u)}\right\}=\beta_{j, k}^{(u)}+O\left(T^{-1}\right)
$$

(b) Agora, queremos obter $\operatorname{Cov}\left\{\hat{\beta}_{j, k}^{(u)}, \hat{\beta}_{j^{\prime}, k^{\prime}}^{\left(u^{\prime}\right)}\right\}$.

Em primeiro lugar, usando (5.7) e (5.9),

$$
\begin{aligned}
& \operatorname{Cov}\left\{\hat{f}_{x x}\left(\frac{t}{T}, \lambda\right), \hat{f}_{x x}\left(\frac{t^{\prime}}{T}, \lambda^{\prime}\right)\right\} \\
& =\operatorname{Cov}\left\{\sum_{\mathcal{I}} \hat{\Theta}_{\mathcal{I}}^{(x x)} \psi_{j_{1}, k_{1}}\left(\frac{t}{T}\right) \tilde{\psi}_{j_{2}, k_{2}}(\lambda), \sum_{\mathcal{I}^{\prime}} \hat{\Theta}_{\mathcal{I}^{\prime}}^{(x x)} \psi_{j_{1}^{\prime}, k_{1}^{\prime}}\left(\frac{t^{\prime}}{T}\right) \tilde{\psi}_{j_{2}^{\prime}, k_{2}^{\prime}}\left(\lambda^{\prime}\right)\right\} \\
& =\sum_{\mathcal{I}} \sum_{\mathcal{I}^{\prime}} \operatorname{Cov}\left\{\hat{\Theta}_{\mathcal{I}}^{(x x)}, \hat{\Theta}_{\mathcal{I}^{\prime}}^{(x x)}\right\} \psi_{j_{1}, k_{1}}\left(\frac{t}{T}\right) \tilde{\psi}_{j_{2}, k_{2}}(\lambda) \psi_{j_{1}^{\prime}, k_{1}^{\prime}}\left(\frac{t^{\prime}}{T}\right) \tilde{\psi}_{j_{2}^{\prime}, k_{2}^{\prime}}\left(\lambda^{\prime}\right) \\
& =\sum_{\mathcal{I}=\mathcal{I}^{\prime}} \operatorname{Var}\left\{\hat{\Theta}_{\mathcal{I}}^{(x x)}\right\} \psi_{j_{1}, k_{1}}\left(\frac{t}{T}\right) \tilde{\psi}_{j_{2}, k_{2}}(\lambda) \psi_{j_{1}, k_{1}}\left(\frac{t^{\prime}}{T}\right) \tilde{\psi}_{j_{2}, k_{2}}\left(\lambda^{\prime}\right) \\
& +\sum_{\mathcal{I} \neq \mathcal{I}^{\prime}} \operatorname{Cov}\left\{\hat{\Theta}_{\mathcal{I}}^{(x x)}, \hat{\Theta}_{\mathcal{I}^{\prime}}^{(x x)}\right\} \psi_{j_{1}, k_{1}}\left(\frac{t}{T}\right) \tilde{\psi}_{j_{2}, k_{2}}(\lambda) \psi_{j_{1}^{\prime}, k_{1}^{\prime}}\left(\frac{t^{\prime}}{T}\right) \tilde{\psi}_{j_{2}^{\prime}, k_{2}^{\prime}}\left(\lambda^{\prime}\right) \\
& =\sum_{\mathcal{I}} C_{\mathcal{I}}^{(x x)} \Psi_{\mathcal{I}}+O\left(T^{-\delta}\right),
\end{aligned}
$$

onde

$$
C_{\mathcal{I}}^{(x x)}=\frac{2 \pi}{T} \int_{U \times \Pi}\left\{f_{x x}(u, \lambda) \psi_{j_{1}, k_{1}}(u)\right\}^{2} d u \tilde{\psi}_{j_{2}, k_{2}}(\lambda)\left[\tilde{\psi}_{j_{2}, k_{2}}(\lambda)+\tilde{\psi}_{j_{2}, k_{2}}(-\lambda)\right] d \lambda
$$


e

$$
\Psi_{\mathcal{I}}=\psi_{j_{1}, k_{1}}\left(\frac{t}{T}\right) \psi_{j_{1}, k_{1}}\left(\frac{t^{\prime}}{T}\right) \tilde{\psi}_{j_{2}, k_{2}}(\lambda) \tilde{\psi}_{j_{2}, k_{2}}\left(\lambda^{\prime}\right)
$$

Daí,

$$
\operatorname{Cov}\left\{\hat{f}_{x x}\left(\frac{t}{T}, \lambda\right), \hat{f}_{x x}\left(\frac{t^{\prime}}{T}, \lambda^{\prime}\right)\right\}=\sum_{\mathcal{I}} C_{\mathcal{I}}^{(x x)} \Psi_{\mathcal{I}}+O\left(T^{-\delta}\right), \quad \text { para } \delta>0 .
$$

Analogamente

$$
\operatorname{Cov}\left\{\hat{f}_{x y}\left(\frac{t}{T}, \lambda\right), \hat{f}_{x y}\left(\frac{t^{\prime}}{T}, \lambda^{\prime}\right)\right\}=\sum_{\mathcal{I}} C_{\mathcal{I}}^{(x y)} \Psi_{\mathcal{I}}+O\left(T^{-\delta}\right), \quad \text { para } \delta>0,
$$

onde

$$
C_{\mathcal{I}}^{(x y)}=\frac{2 \pi}{T} \int_{U \times \Pi}\left\{f_{x y}(u, \lambda) \psi_{j_{1}, k_{1}}(u)\right\}^{2} d u \tilde{\psi}_{j_{2}, k_{2}}(\lambda)\left[\tilde{\psi}_{j_{2}, k_{2}}(\lambda)+\tilde{\psi}_{j_{2}, k_{2}}(-\lambda)\right] d \lambda .
$$

Usando a expansão de Taylor, obtemos

$$
\begin{aligned}
& \operatorname{Cov}\left\{\hat{B}_{a}\left(\frac{t}{T}, \frac{p}{P_{T}}\right), \hat{B}_{a}\left(\frac{t^{\prime}}{T}, \frac{p^{\prime}}{P_{T}}\right)\right\} \\
& =\operatorname{Cov}\left\{\frac{\hat{f}_{x y}\left(\frac{t}{T}, \frac{p}{P_{T}}\right)}{\hat{f}_{x x}\left(\frac{t}{T}, \frac{p}{P_{T}}\right)}, \frac{\hat{f}_{x y}\left(\frac{t^{\prime}}{T}, \frac{p^{\prime}}{P_{T}}\right)}{\hat{f}_{x x}\left(\frac{t^{\prime}}{T}, \frac{p^{\prime}}{P_{T}}\right)}\right\} \\
& =\frac{1}{f_{x x}\left(\frac{t}{T}, \frac{p}{P_{T}}\right) f_{x x}\left(\frac{t^{\prime}}{T}, \frac{p^{\prime}}{P_{T}}\right)}\left\{\operatorname{Cov}\left[\hat{f}_{x y}\left(\frac{t}{T}, \frac{p}{P_{T}}\right), \hat{f}_{x y}\left(\frac{t^{\prime}}{T}, \frac{p^{\prime}}{P_{T}}\right)\right]\right. \\
& -B_{a}\left(\frac{t}{T}, \frac{p}{P_{T}}\right) \operatorname{Cov}\left[\hat{f}_{x y}\left(\frac{t^{\prime}}{T}, \frac{p^{\prime}}{P_{T}}\right), \hat{f}_{x x}\left(\frac{t}{T}, \frac{p}{P_{T}}\right)\right] \\
& -B_{a}\left(\frac{t^{\prime}}{T}, \frac{p^{\prime}}{P_{T}}\right) \operatorname{Cov}\left[\hat{f}_{x y}\left(\frac{t}{T}, \frac{p}{P_{T}}\right), \hat{f}_{x x}\left(\frac{t^{\prime}}{T}, \frac{p^{\prime}}{P_{T}}\right)\right] \\
& \left.+B_{a}\left(\frac{t}{T}, \frac{p}{P_{T}}\right) B_{a}\left(\frac{t^{\prime}}{T}, \frac{p^{\prime}}{P_{T}}\right) C o v\left[\hat{f}_{x x}\left(\frac{t}{T}, \frac{p}{P_{T}}\right), \hat{f}_{x x}\left(\frac{t^{\prime}}{T}, \frac{p^{\prime}}{P_{T}}\right)\right]\right\}+O\left(T^{-\delta}\right) \\
& =\frac{1}{f_{x x}\left(\frac{t}{T}, \frac{p}{P_{T}}\right) f_{x x}\left(\frac{t^{\prime}}{T}, \frac{p^{\prime}}{P_{T}}\right)}\left\{\sum_{\mathcal{I}} C_{\mathcal{I}}^{(x y)} \Psi_{\mathcal{I}}+\sum_{\mathcal{I}} C_{\mathcal{I}}^{(x x)} \Psi_{\mathcal{I}} B_{a}\left(\frac{t}{T}, \frac{p}{P_{T}}\right) B_{a}\left(\frac{t^{\prime}}{T}, \frac{p^{\prime}}{P_{T}}\right)\right\}+O\left(T^{-\delta}\right) .
\end{aligned}
$$


Finalmente,

$$
\begin{aligned}
& \operatorname{Cov}\left\{\hat{\beta}_{j, k}^{(u)}, \hat{\beta}_{j^{\prime}, k^{\prime}}^{\left(u^{\prime}\right)}\right\} \\
& =\frac{1}{T^{2}} \sum_{t, t^{\prime}} \frac{1}{P_{T}^{2}} \sum_{p, p^{\prime}} \exp \left[i\left(u \frac{p}{P_{T}}+u^{\prime} \frac{p^{\prime}}{P_{T}}\right)\right] \operatorname{Cov}\left[\hat{B}_{a}\left(\frac{t}{T}, \frac{p}{P_{T}}\right), \hat{B}_{a}\left(\frac{t^{\prime}}{T}, \frac{p^{\prime}}{P_{T}}\right)\right] \psi_{j, k}\left(\frac{t}{T}\right) \psi_{j^{\prime}, k^{\prime}}\left(\frac{t^{\prime}}{T}\right),
\end{aligned}
$$

de onde obtemos (5.26).

(c) Agora queremos obter a distribuição assintótica de $\hat{\beta}_{j, k}^{(u)}$.

Em primeiro lugar, obtemos

$$
\begin{aligned}
& \left|\operatorname{Cum}_{n}\left(\hat{f}_{x x}(u, \lambda)\right)\right| \\
& =\left|\operatorname{Cum}\left\{\sum_{\mathcal{I}_{1}} \hat{\Theta}_{\mathcal{I}_{1}}^{(x x)} \psi_{j_{11}, k_{11}}\left(u_{1}\right) \tilde{\psi}_{j_{21}, k_{21}}\left(\lambda_{1}\right), \cdots, \sum_{\mathcal{I}_{n}} \hat{\Theta}_{\mathcal{I}_{n}}^{(x x)} \psi_{j_{1 n}, k_{1 n}}\left(u_{n}\right) \tilde{\psi}_{j_{2 n}, k_{2 n}}\left(\lambda_{n}\right)\right\}\right| \\
& =\left|\sum_{\mathcal{I}_{1}} \cdots \sum_{\mathcal{I}_{n}} \operatorname{Cum}\left\{\hat{\Theta}_{\mathcal{I}_{1}}^{(x x)}, \cdots, \hat{\Theta}_{\mathcal{I}_{n}}^{(x x)}\right\} \psi_{j_{11}, k_{11}}\left(u_{1}\right) \tilde{\psi}_{j_{21}, k_{21}}\left(\lambda_{1}\right) \cdots \psi_{j_{1 n}, k_{1 n}}\left(u_{n}\right) \tilde{\psi}_{j_{2 n}, k_{2 n}}\left(\lambda_{n}\right)\right| \\
& \leq\left|\operatorname{Cum}_{n}\left(\hat{\Theta}_{\mathcal{I}}^{(x x)}\right)\right|\left|\sum_{\mathcal{I}} \psi_{j_{1}, k_{1}}(u) \tilde{\psi}_{j_{2}, k_{2}}(\lambda)\right|^{n} \\
& \leq\left|\operatorname{Cum}_{n}\left(\hat{\Theta}_{\mathcal{I}}^{(x x)}\right)\right|\left(\sum_{\mathcal{I}} 2^{j_{1} / 2} A_{1} 2^{j_{2} / 2} A_{2}\right)^{n} \\
& \leq(n !)^{2+2 \gamma} C^{n} T^{-\delta}\left(T^{(-1-\delta) / 2} 2^{\left(j_{1}+j_{2}\right) / 2} \log (T)\right)^{n-2}, \quad \text { para } n \geq 3 .
\end{aligned}
$$

Assim,

$$
\operatorname{Cum}_{n}\left(\hat{f}_{x x}(u, \lambda)\right) \rightarrow 0,
$$

quando $T \rightarrow \infty$, para $n \geq 3$.

Analogamente,

$$
\operatorname{Cum}_{n}\left(\hat{f}_{x y}(u, \lambda)\right) \rightarrow 0
$$

quando $T \rightarrow \infty$, para $n \geq 3$.

Daí, quando $T \rightarrow \infty$, coleções finitas de $\hat{f}_{x x}(u, \lambda), \hat{f}_{x y}(u, \lambda)$ são assintoticamente normais e normais complexas, respectivamente.

Agora, como temos

$$
\hat{\beta}_{j, k}^{(u)}=\frac{1}{T} \frac{1}{P_{T}} \sum_{t=0}^{T-1} \sum_{p=0}^{P_{T}-1} \frac{\hat{f}_{x y}\left(\frac{t}{T}, \frac{p}{P_{T}}\right)}{\hat{f}_{x x}\left(\frac{t}{T}, \frac{p}{P_{T}}\right)} \exp \left(i u \frac{p}{P_{T}}\right) \psi_{j, k}\left(\frac{t}{T}\right),
$$


então, pelos lemas 5.2 e 5.3 e pela demonstração do teorema 8.10.1 de Brillinger (1975), obtemos o resultado (c).

O próximo resultado é imediato.

Teorema 5.2: Assumindo suposições (S1) a (S9), temos

(a)

$$
E\left\{\tilde{a}_{u}\left(\frac{s}{T}\right)\right\}=\sum_{j} \sum_{k} \beta_{j, k}^{(u)} \psi_{j, k}\left(\frac{s}{T}\right)+O\left(2^{J_{T} / 2} T^{-1}\right)
$$

(b)

$$
\begin{aligned}
& \operatorname{Cov}\left\{\tilde{a}_{u}\left(\frac{s}{T}\right), \tilde{a}_{u^{\prime}}\left(\frac{s^{\prime}}{T}\right)\right\}= \\
& \quad \frac{1}{T^{2}} \sum_{t, t^{\prime}=0}^{T-1} \frac{1}{P_{T}^{2}} \sum_{p, p^{\prime}=0}^{P_{T}-1} \frac{\sum_{\mathcal{I}}\left[C_{\mathcal{I}}^{(x y)}+C_{\mathcal{I}}^{(x x)} B_{a}\left(\frac{t}{T}, \frac{p}{P_{T}}\right) B_{a}\left(\frac{t^{\prime}}{T}, \frac{p^{\prime}}{P_{T}}\right)\right] \Psi_{\mathcal{I}}}{f_{x x}\left(\frac{t}{T}, \frac{p}{P_{T}}\right) f_{x x}\left(\frac{t^{\prime}}{T}, \frac{p^{\prime}}{P_{T}}\right)} \exp \left[i\left(u \frac{p}{P_{T}}+u^{\prime} \frac{p^{\prime}}{P_{T}}\right)\right] \\
& \sum_{j, j^{\prime}} \sum_{k, k^{\prime}} \psi_{j, k}\left(\frac{s}{T}\right) \psi_{j^{\prime}, k^{\prime}}\left(\frac{s^{\prime}}{T}\right) \psi_{j, k}\left(\frac{t}{T}\right) \psi_{j^{\prime}, k^{\prime}}\left(\frac{t^{\prime}}{T}\right)+O\left(2^{J_{T}} T^{-2-\delta}\right),
\end{aligned}
$$

para algum $\delta>0$. Em (5.34),

$$
\begin{gathered}
C_{\mathcal{I}}^{(x x)}=\frac{2 \pi}{T} \int_{U \times \Pi}\left\{f_{x x}(u, \lambda) \psi_{j_{1}, k_{1}}(u)\right\}^{2} \tilde{\psi}_{j_{2}, k_{2}}(\lambda)\left[\tilde{\psi}_{j_{2}, k_{2}}(\lambda)+\tilde{\psi}_{j_{2}, k_{2}}(-\lambda)\right] d u d \lambda, \\
C_{\mathcal{I}}^{(x y)}=\frac{2 \pi}{T} \int_{U \times \Pi}\left\{f_{x y}(u, \lambda) \psi_{j_{1}, k_{1}}(u)\right\}^{2} \tilde{\psi}_{j_{2}, k_{2}}(\lambda)\left[\tilde{\psi}_{j_{2}, k_{2}}(\lambda)+\tilde{\psi}_{j_{2}, k_{2}}(-\lambda)\right] d u d \lambda, \\
\Psi_{\mathcal{I}}=\psi_{j_{1}, k_{1}}\left(\frac{t}{T}\right) \psi_{j_{1}, k_{1}}\left(\frac{t^{\prime}}{T}\right) \tilde{\psi}_{j_{2}, k_{2}}\left(\frac{p}{P_{T}}\right) \tilde{\psi}_{j_{2}, k_{2}}\left(\frac{p^{\prime}}{P_{T}}\right),
\end{gathered}
$$

$\operatorname{com} \sum_{\mathcal{I}} C_{\mathcal{I}}^{(x x)} \Psi_{\mathcal{I}}=O\left(T^{-\delta}\right)$ e $\sum_{\mathcal{I}} C_{\mathcal{I}}^{(x y)} \Psi_{\mathcal{I}}=O\left(T^{-\delta}\right)$; os erros em (5.33) e (5.34) são uniformes em $\mathcal{I}$.

(c) $\tilde{a}_{u}\left(\frac{s}{T}\right)$ tem uma distribuição assintoticamente normal, com média e a estrutura de covariância dadas por (5.33) and (5.34), respectivamente.

Observação: Para melhorar o estimador $\tilde{a}_{u}\left(\frac{s}{T}\right)$ definido em (5.24), propomos usar o estimador de encolhimento não-linear

$$
\tilde{a}_{u}^{*}\left(\frac{s}{T}\right)=\sum_{j=0}^{J_{T}} \sum_{k} \delta^{(\cdot)}\left(\hat{\beta}_{j, k}^{(u)}, \lambda_{j}^{(u)}\right) \psi_{j, k}\left(\frac{s}{T}\right),
$$


onde $\lambda_{j}^{(u)}=\sigma_{j} \sqrt{\log T}$ e $\delta^{(\cdot)}$ pode ser uma política dura ou suave. Aqui, $\sigma_{j}$ é o desvio padrão dos coeficientes $\hat{\beta}_{j, k}^{(u)}$ no nível $j$. Propriedades análogas às vistas acima valem para $\tilde{a}_{u}^{*}\left(\frac{s}{T}\right)$.

\subsection{Aplicação}

Como um exemplo investigaremos as relações entre a série, $P(t)$, de produção de carros e a série, $E(t)$, de exportação de carros, na Espanha, de janeiro de 1980 a junho de 1996 (dados cedidos por Miguel A. Arino, Universidade de Navarra, Barcelona). Como essas séries apresentam comportamentos sazonais anuais, antes de fazer análise, dessazonalisamo-as. Se $Y(t)$ denota a série ajustada de $E(t)$, então ela é obtida por

$$
Y(t-12)=E(t)-E(t-12), \quad t=13,14, \cdots
$$

Da mesma maneira, obtemos $X(t)$, a série ajustada de $P(t)$,

$$
X(t-12)=P(t)-P(t-12), \quad t=13,14, \cdots
$$

Essas séries são dadas nas Figuras C.3 e C.4, e as séries originais são dadas nas Figuras C.1 e C.2.

Devido a problemas computacionais, tomamos $T=128$ para nossa análise e determinamos diversas estatísticas apresentadas na seção 5.3. Os resultados são registrados numa série de figuras. Aqui, a ondaleta "Daublet" $d 4$ é usada como base.

A Figura C.5 é o periodograma de $X(t), I_{t, T}^{(x x)}(\lambda)$ e as Figuras C.6 e C.7 são de parte real e parte imaginaria do periodograma cruzado entre $X(t)$ e $Y(t)$, $\operatorname{Re} I_{t, T}^{(x y)}(\lambda), \operatorname{Im} I_{t, T}^{(x y)}(\lambda)$, respectivamente. Os espectros $\hat{f}_{x x}\left(\frac{t}{T}, \lambda\right), \operatorname{Re} \hat{f}_{x y}\left(\frac{t}{T}, \lambda\right)$ e $\operatorname{Im} \hat{f}_{x y}\left(\frac{t}{T}, \lambda\right)$ são apresentados nas Figuras C. 8 , C.9 e C.10, respectivamente, cujas imagens são apresentadas nas Figuras C.11, C.12 e C.13. As estimativas dos coeficientes do filtro $a_{u}\left(\frac{t}{T}\right)$ para $|u| \leq 20$ são dadas na Figura C.14 e as respectivas imagens na Figura C.15. 


\section{Discussões e Conclusões}

O objetivo deste trabalho foi a utilização de ondaletas na análise de alguns aspectos de processos estacionários e localmente estacionários.

Ondaleta é uma técnica nova que foi introduzida na área de estatística recentemente. Para processos estacionários não havia na literatura uma análise espectral (em tempo e escala) via ondaletas. Neste trabalho, o conceito do espectro de ondaleta foi introduzido e as propriedades assintóticas das transformadas de ondaleta foram obtidas. O periodograma de ondaleta foi definido e suas propriedades assintóticas foram estudadas.

A nossa contribuição para processos localmente estacionários foi a apresentação de um método de estimação de sistemas lineares variando no tempo. Nesta parte, utilizarmos uma expansão dos coeficientes do filtro do sistema em ondaletas para estimá-los. A seguir, as propriedades assintóticas destes estimadores foram estudadas.

A literatura para análise de ondaletas em séries temporais ainda é muito limitada apesar da alta velocidade de progresso. Trabalhos futuros envolvem a obtenção das propriedades assintóticas dos estimadores de encolhimento não linear definidos em (4.14) e (5.35) para processos estacionários e localmente estacionários, respectivamente. Além disso, podemos usar ondaletas para tratar de vários modelos, como: modelos de espaços de estados, modelos lineares generalizados (em particular envolvendo efeitos aleatórios), modelos com componentes sazonais, etc. Outro uso seria no estado de processos pontuais (em particular o processo de Poisson). 


\section{Referências}

Arino, M.A. and Vidakovic, B. (1995). On wavelet scalograms and their applications in economic time series. Preprint, ISDS Working Papers, Duke University.

Blackman, R.B. and Tukey, J.W. (1959). The Measurement of Power Spectra. Dover, NY.

Brillinger, D.R. (1975). Time Series: Data Analysis and Theory. Holt, Rinehart and Winston.

Brillinger, D.R. (1994). Some river wavelets. Environmetrics, 5, 211-220.

Brillinger, D.R. (1996). Some uses of cumulant in wavelet analysis. Nonparametric Statistics, 6, 93-114.

Brockwell, P.J. and Davis, R.A. (1991). Time Series: Theory and Methods. Second Edition. New York: Springer-Verlag.

Bruce, A.G. and Gao, H.-Ye (1995). Understanding waveshrink: Variance and bias estimation. A aparecer, Biometrika.

Bruce, A.G. and Gao, H.-Ye (1996). Applied Wavelet Analysis with S-PLUS. New York: Springer.

Buckheit, J.B. and Donoho, D.L. (1995). Improved linear discrimination using time frequency dictionaries. TR, Stanford University.

Chui, C.K. (1992). An Introduction to Wavelets. San Diego: Academic Press.

Cooley, J.W. and Tukey, J.W. (1965). An algorithm for the machine calculation of complex Fourier series. Math. Comp., 19, 297-301.

Dahlhaus, R. (1996). Asymptotic statistical inference for nonstationary processes with evolutionary spectra. In Athens Conference on Applied Probability and Time Series Analysis, Vol II (P.M. Robinson and M. Rosenblatt, eds.). New York: Springer-Verlag.

Dahlhaus, R. (1997). Fitting time series models to nonstationary processes. Ann. Statist., 25, 1-37. 
Daubechies, I. (1992) Ten Lectures on Wavelets. CBMS Lecture Notes Series. Philadelphia: SIAM.

Delyon, B. and Juditsky, A. (1993). Wavelet estimators, global error measures: Revisited. TR 782, IRISA-INRIA.

Donoho, D.L. (1991). Nonlinear solution of linear inverse problems by wavelet-vaguelette decomposition. TR, Department of Statistics, Stanford University.

Donoho, D.L. (1993). Nonlinear wavelet methods for recovery of signals, densities, and spectra from indirect and noisy data. Proceedings of Symposia in Applied Mathematics, 47, 173-205, AMS.

Donoho, D.L. (1995). De-noising by soft thresholding. IEEE Transactions on Information Theory, 41(3), 613-627.

Donoho, D.L. and Johnstone, I.M. (1990). Wavelets and optimal nonlinear function estimates. TR 281, Statistics Department, University of California, Berkeley.

Donoho, D.L. and Johnstone, I.M. (1994). Ideal spatial adaptation by wavelet shrinkage. Biometrika, 81, 425-455.

Donoho, D.L. and Johnstone, I.M. (1995a). Minimax estimation via wavelet shrinkage. Submitted to the Annals of Statistics.

Donoho, D.L. and Jonhstone, I.M. (1995b). Adapting to unknown smoothness via wavelet shrinkage. Journal of the American Statistical Association, 90, 1200-1224.

Donoho, D.L., Jonhstone, I.M., Kerkyacharian, G. and Picard, D. (1995). Wavelet shrinkage: asymptopia?(with discussion). Journal of the Royal Statistical Society, Series B, 57, 301-369.

Donoho, D.L., Jonhstone, I.M., Kerkyacharian, G. and Picard, D. (1996). Universal near minimaxity of wavelet shrinkage. In Festschrift for Lucien Le Cam(D.Pollard and G. Yang, eds.). Springer-Verlag.

Farge, M. (1992). Wavelet transforms and their applications to turbulence. Annu. Rev. Fluid Mech., 24, 395-457. 
Flandrin, P. (1989). Time dependent spectra for nonstationary stochastic processes. In Time and Frequency Representations of Signals and Systems (G.Longo and B.Picinbono, eds.), 69-124. New York: SpringerVerlag.

Fuller, W.A. (1996). Introduction to Statistical Time Series. Second Edition. New York: John Wiley and Sons.

Gao, H.-Y. (1993). Wavelet estimation of spectral densities in time series analysis. Ph.D. thesis, Univ.of California, Berkeley.

Gao, H.-Y. (1997). Choice of thresholds for wavelet shrinkage estimate of the spectrum. A aparecer, Journal of Time Series Analisis.

Grossmann, A. and Morlet, J. (1984). Decomposition of Hardy functions into square integrable wavelets of constant shape. SIAM J. Math. Anal., $15,723-736$.

Hall, P. and Patil, P. (1993a). On wavelet methods for estimating smooth functions. TR 12-93, Center for Mathematics and its Applications, Australian National University.

Hall, P. and Patil, P. (1993b). Formulas for mean integrated squared error of nonlinear wavelet based density estimation. TR 15-93, Center for Mathematics and its Applications, Australian National University.

Hall, P. and Patil, P. (1993c). On the choice of smoothing parameter, thresold and truncation in nonparametric regression by nonlinear wavelet methods. TR 21-93, Center for Mathematics and its Applications, Australian National University.

Hannan, E.J. (1970). Multiple Time Series. New York: Wiley.

Hannan, E.J. (1973). Central limit theorems for time series regression. Zeitshrift fur Wahrscheinlichkeits Theorie and Gebiet, 26, 157-170.

Johnstone, I.M. and Silverman, B. (1997). Wavelet threshold estimators for data with correlated noise. TR, Stanford University.

Johnstome, I.M., Kerkyacharian, G. and Picard, D, (1992). Estimation d'une densité de probabilité par méthode d'ondelettes. Comptes Rendus Acad. Sciences Paris (A), 315, 211-216. 
King, N. (1972). An alternative for the linear regression equation when the predictor variable is uncontrolled and the sample size is small. Journal of the American Statistical Association, 67, 217-219.

Kolaczyk, E. (1994). WVD Solution of Inverse Problems. Phd thesis, Stanford University.

Lina, J.M. and Mayrand, M. (1995). Image enhancement with symmetric Daubechies wavelets. In proceedings of the SPIE 1995 Symposium, Wavelet Applications in Signal and Image processing, San Diego, CA, July.

Mallat, S. (1989). A theory for multiresolution signal decompositions: the wavelet representation. IEEE Trans. on Pattern Anal. Machine Intell., 11, 674-693.

Mardia, K.V., Kent, J.T. and Bibby, J.M. (1979). Multivariate Analysis. London: Academic Press.

Meyer, Y. (1990). Ondelettes et Opérateurs. Tome I. Actualités Mathématiques. Paris: Hermann.

Meyer, Y. (1993). Wavelets: Algorithms and Applications. Philadelphia: SIAM.

Miller, E.L. and Willsky, A.S. (1994). Wavelet transforms and multiscale estimation techniques for the solution of multisensor inverse problems. In SPIE Proceedings, Wavelet Applications, Vol. 2242, Orlando, FL, April.

Morettin, P.A. (1981). Walsh spectral analysis. SIAM Review, 23, 279-291.

Morettin, P.A. (1996). From Fourier to wavelet analysis of time series. In Proceedings in Computational Statistics (A.Prat, editor), 111-122. Physica-Verlag.

Moulin, P. (1992). Wavelets as a regularization technique for spectral density estimation. In Proc. IEEE Signal Processing Symposium on TimeFrequency and Time-Scale Analysis, October, 73-76.

Moulin, P. (1994). Wavelet thresholding techniques for power spectrum estimation. IEEE Trans. on Signal Processing, 42, 3126-3136. 
Nason, G.P. (1995). Wavelet function estimation using cross-validation. In Wavelets and Statistics, 261-280 (Antoniadis, A. and Oppenheim, G., editors). New York, Springer-Verlag. Lecture Notes in Statistics 103.

Nason, G.P. and Silverman, B.W. (1994). The discrete wavelet transform in S. Journal of Computational and Graphical Statistics, 3, 163-191.

Neumann, M.H. (1996). Spectral density estimation via nonlinear wavelet methods for stationary non-Gaussian time series. Journal Time Series Analysis, 17, 601-633.

Neumann, M.H. and von Sachs, R. (1995). Wavelet thresholding: Beyond the gaussian I.I.D. situation. In Wavelets and Statistics (Anestis Antoniadis and Georges Oppenheim, editors), 301-329. New York: SpringerVerlag.

Neumann, M.H. and von Sachs, R. (1997). Wavelet thresholding in anisotropic function classes and application to adaptive estimation of evolutionary spectra. Ann. Statist., 25, 38-76.

Page, C.H. (1952). Instantaneous power spectra. J. Appl. Phys., 23, 103106.

Parzen, E. (1959). Statistical inference on time series by Hilbert space methods I. In Time Series Analysis Papers. Holden-Day, San Francisco.

Percival, D.B. and Walden, A.T. (1993). Spectral Analysis for Physical Application: Multitaper and Conventional Univariate Techniques. Cambridge: Cambridge University Press.

Percival, D.B. (1993). An introduction to spectral analysis and wavelets. In proceedings of the Workshop, Applied Mathematical Tools in Metrology, Torino, Italy, October.

Polya, G. and Szegö, G. (1925). Aufgaben und Lehrsaetze aus der Analysis I. Berlin: Springer.

Priestley, M.B. (1965). Evolutionary spectra and nonstationary processes. J. Roy. Statist. Soc., Series B, 27, 204-237.

Priestley, M.B. (1981). Spectral Analysis and Time Series. London: Academic Press. 
Priestley, M.B. (1996). Wavelets and time dependent spectral analysis. Journal Time Series Analysis, 17, 85-103.

Robinson, P.M. (1979). Distributed lag approximation to linear time-invariant systems. The Annals of Statistics, Vol. 7, 507-515.

Saito, N. and Coifman, R. (1994). Local discriminant bases. In SPIE Proceedings, Wavelet Applications, Vol. 2303, San Diego, CA, July.

Scargle, J.D. (1993). Wavelet methods in astronomical time series. In Applications of Time Series in Astronomy and Meteorology (ed. T.Subba Rao and O.Lessie). London: Chapman and Hall.

Stoffer, D.S., Scher, M.S., Richardson, G.A., Day, N.L. and Coble, P.A. (1988). A Walsh-Fourier analysis of the effects of moderate maternal alcohol consumption on neonatal sleep state cycling. Journal of the American Statistical Association, 83, 954-963.

Stoffer, D.S. (1991). Walsh-Fourier analysis and its statistical applications. Journal of the American Statistical Association, 86, 461-485.

Strang, G. (1989). Wavelets and dilation equations. SIAM Review, 31, 614-627.

Thompson, J.R. (1968). Some shrinkage techniques for estimating the mean. Journal of the American Statistical Association, 63, 113-122.

Tjøstheim, D. (1976). Spectral generating operators for non-stationary processes. Adv. Appl. Prob., 8, 831-846.

Tribouley, K. (1995). Adaptive density estimation. In Wavelets and Statistics (Anestis Antoniadis and Georges Oppenheim, editors), 385-395. New York: Springer-Verlag.

von Sachs, R. (1996). Modelling and estimation of time-varying structure of nonstationary time series. A aparecer, REBRAPE - Brazilian J.Probab. and Statistics.

von Sachs, R. and Schneider, K. (1996). Wavelet smoothing of evolutionary spectra by non-linear thresholding. Appl. Comput. Harmonic Analysis, 3, 268-283. 
von Sachs, R. Nason, G.P. and Kroisandt. G. (1996). Spectral representation and estimation for locally stationary wavelet processes. In Proc. Workshop on Spline Functions and Theory of Wavelets. Montréal, Canada, 1996.

Vannucci, M. and Vidakovic, B. (1995). Preventing the dirac disaster: Wavelet based density estimation. Discussion Paper 27, Duke University.

Walden, A.T., McCoy, E.J. and Percival, D.B. (1995). Spectrum estimation by wavelet thresholding of multitaper estimators. TR, Imperial College of Science, Technology and Medicine, August.

Wang, Y. (1995). Jump and sharp cusp detection by wavelets. Biometrika, 8292, 385-397.

Wickerhauser, M.V. (1994). Wavelet approximations to Jacobians and the inversion of complicated maps. In proceedings of the SPIE, Wavelet Applications, Vol. 2242, Orlando, FL.

Xia, X.-G., Kuo, C.-C.J. and Suter, B.W. (1994). Improved Backus-Gilbert method for signal reconstruction with a wavelet model. In SPIE Proceedings, Wavelet Applications, Vol. 2242, Orlando, FL. April. 


\section{Apêndice A}

\section{Funções de Ondaletas Ortogonais}

Este apêndice apresenta algumas famílias de ondaletas ortogonais. As "Daublets" estão nas Figuras A.1 e A.2, as "Coiflets" nas Figuras A.3 e as "Symmlets" nas Figuras A.4 e A.5. Algumas propriedades sobre essas funções (o tamanho do suporte, o número de momentos nulos, o número de derivadas) são resumidas na Tabela A.1 

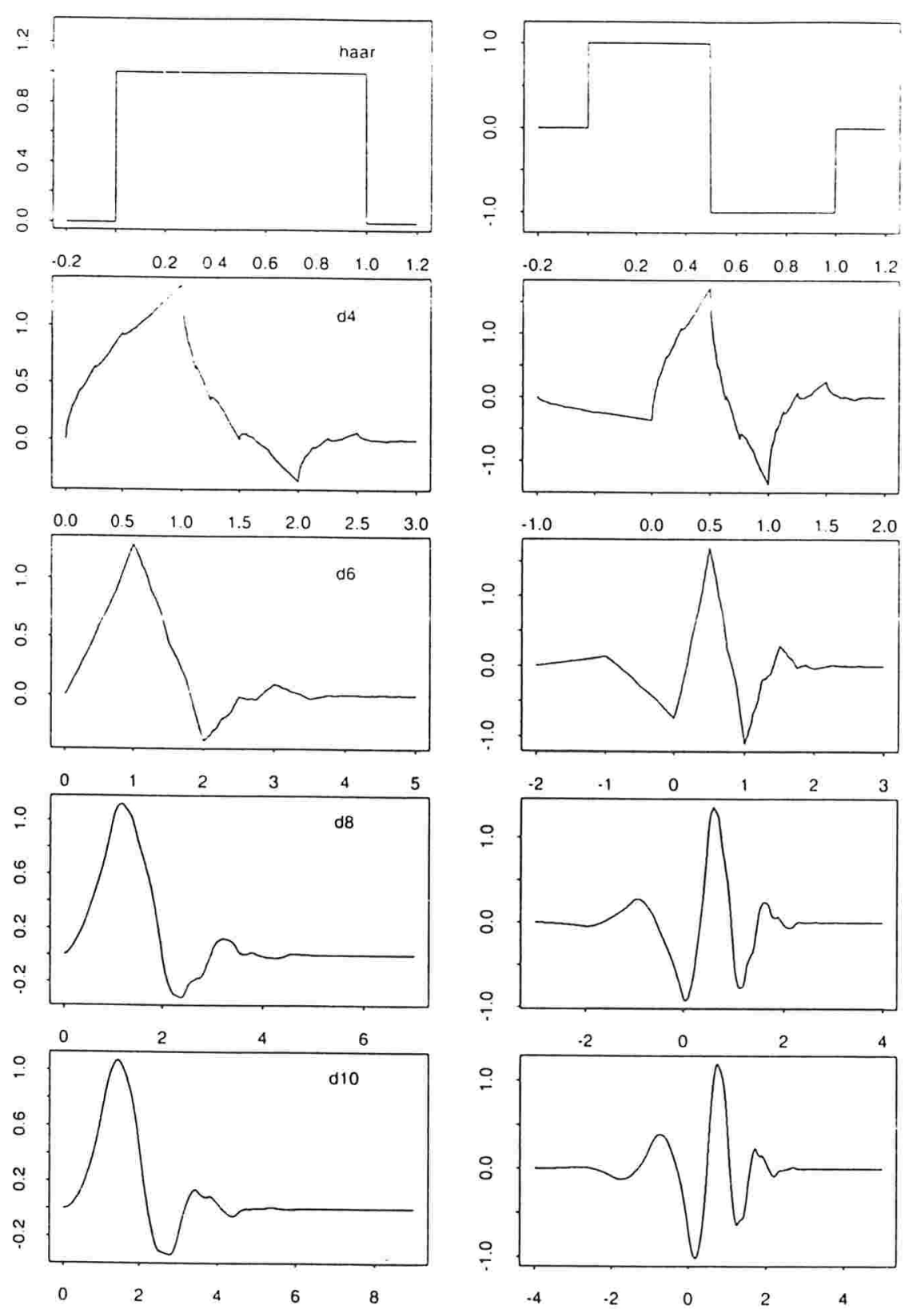

Figura A.1. As ondaletas ortogonais de Haar e "Daublets", haar, d4, d6, d8 e d10. (a) ondaletas "pai". (b) ondaletas "mãe". 

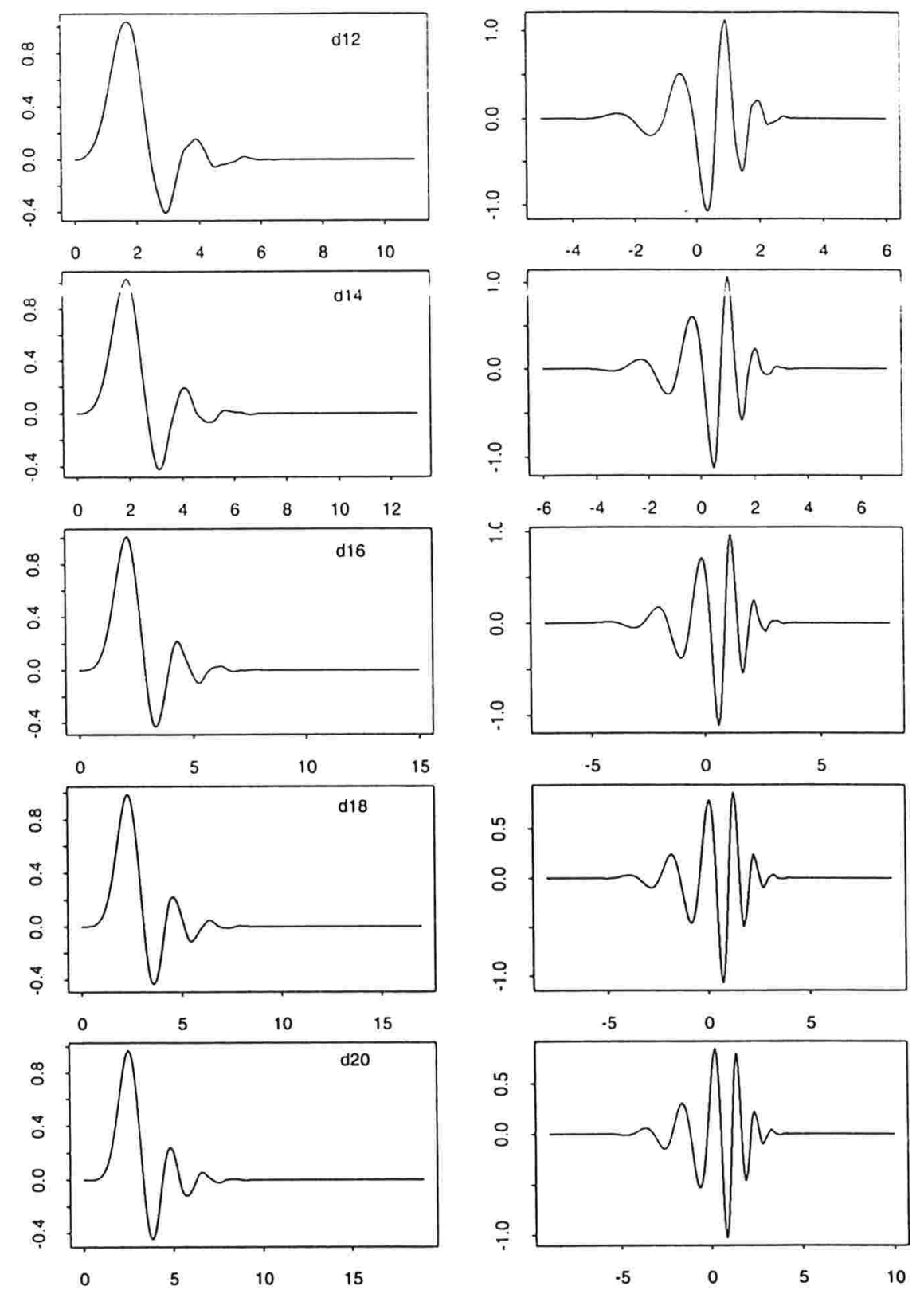

Figura A.2. As ondaletas ortogonais "Daublets" d12, d14, d16, d18 e d20. (a) ondaletas "pai". (b) ondaletas "mãe". 

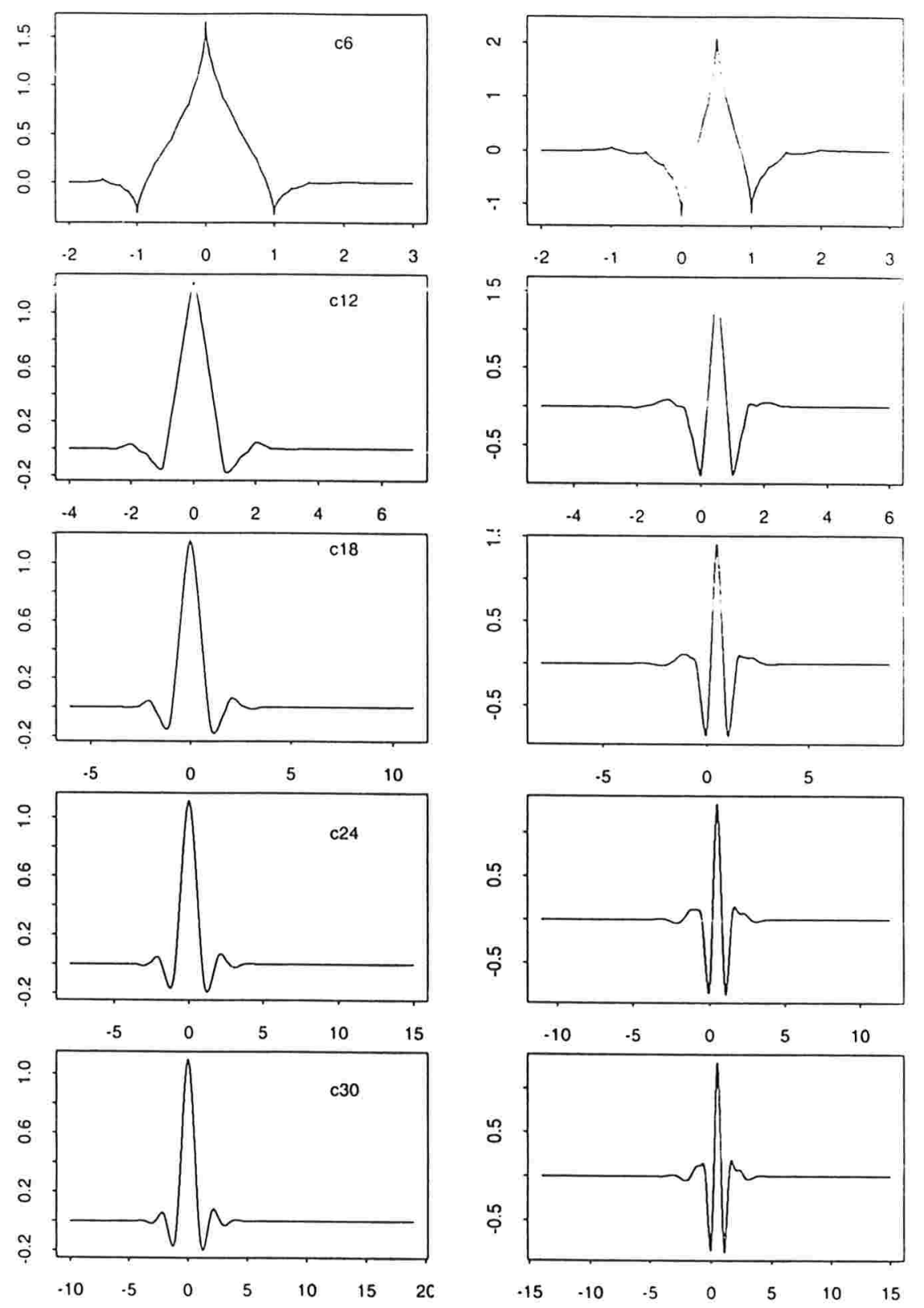

Figura A.3. As ondaletas ortogonais "Coiflets" c6, c12, c18, c24 e c30. (a) ondaletas "pai". (b) ondaletas "mãe". 

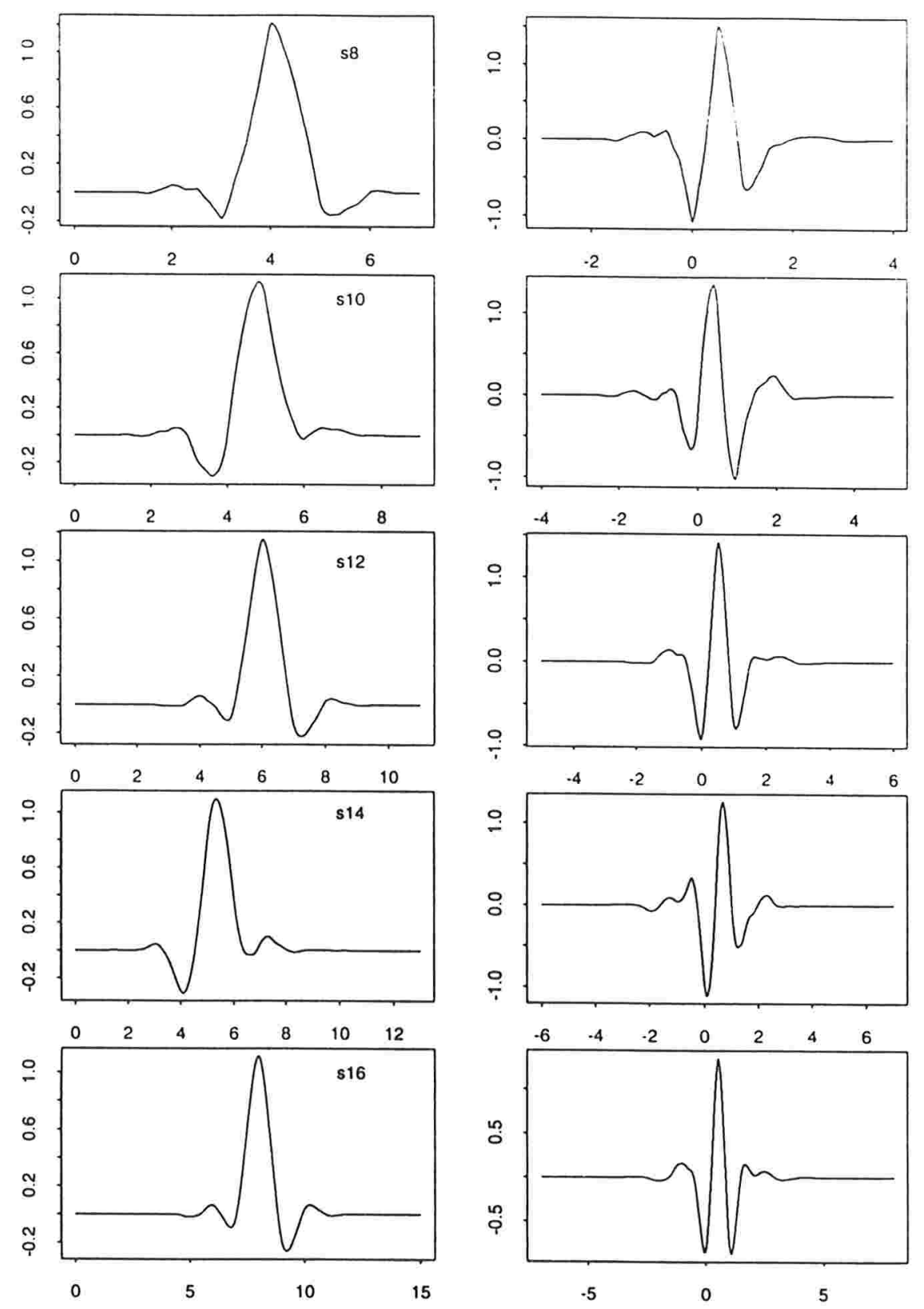

Figura A.4. As ondaletas ortogonais "Symmlets" s8, s10, s12, s14 e s16. (a) ondaletas "pai". (b) ondaletas "mãe". 

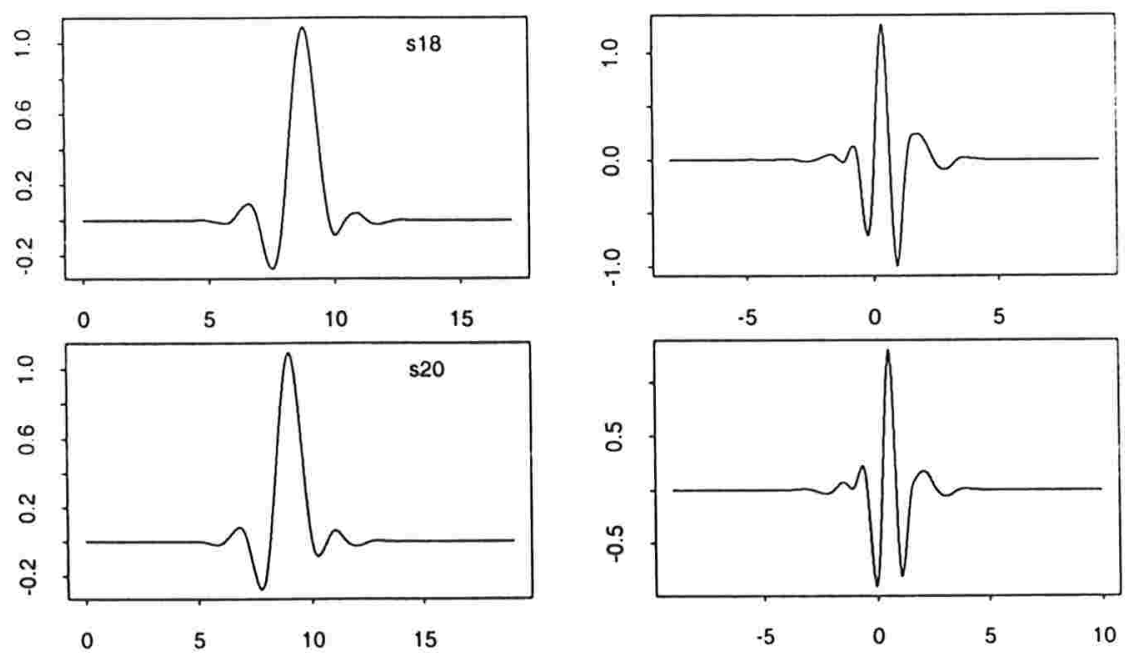

Figura A.5. As ondaletas ortogonais "Symmlets" s18 e s20. (a) ondaletas "pai". (b) ondaletas "mãe". 


\begin{tabular}{|c|c|c|c|c|}
\hline \multirow[b]{2}{*}{ Ondaleta } & $\begin{array}{l}\text { O tamanho } \\
\text { do Suporte }\end{array}$ & \multicolumn{2}{|c|}{$\begin{array}{l}\text { Momentos } \\
\text { Nulos }\end{array}$} & \multirow{2}{*}{$\begin{array}{c}\text { Número de } \\
\text { Derivadas } \\
\psi, \phi\end{array}$} \\
\hline & $\psi, \phi$ & $\psi$ & $\phi$ & \\
\hline haar & 1 & 0 & 0 & 0 \\
\hline $\mathrm{d} 4$ & 3 & 1 & 0 & 0 \\
\hline $\mathrm{d} 6$ & 5 & 2 & 0 & 1 \\
\hline $\mathrm{d} 8, \mathrm{~s} 8$ & 7 & 3 & 0 & 1 \\
\hline $\mathrm{d} 10, \mathrm{~s} 10$ & 9 & 4 & 0 & 1 \\
\hline $\mathrm{d} 12, \mathrm{~s} 12$ & 11 & 5 & 0 & 2 \\
\hline $\mathrm{d} 14, \mathrm{~s} 14$ & 13 & 6 & 0 & 2 \\
\hline $\mathrm{d} 16, \mathrm{~s} 16$ & 15 & 7 & 0 & 2 \\
\hline $\mathrm{d} 18, \mathrm{~s} 18$ & 17 & 8 & 0 & 3 \\
\hline $\mathrm{d} 20, \mathrm{~s} 20$ & 19 & 9 & 0 & 3 \\
\hline c6 & 5 & 1 & 2 & 0 \\
\hline $\mathrm{c} 12$ & 11 & 3 & 4 & 1 \\
\hline c18 & 17 & 5 & 6 & 2 \\
\hline c24 & 23 & 7 & 8 & 2 \\
\hline c30 & 29 & 9 & 10 & 3 \\
\hline
\end{tabular}

Tabela A.1. Ondaletas ortogonais e suas propriedades. 


\section{Apêndice B}

\section{Figuras Citadas no Capítulo 4}

Este apêndice apresenta uma série de figuras citadas no capítulo 4.

\section{Legenda:}

Figura B.1: A função de autocovariância $(\gamma(u))$ e o espectro de ondaleta $\left(\eta_{j, k}^{(H)}\right)$ com respeito a ondaleta de Haar de uma sequência de ruído branco, com média zero e variância $\sigma^{2}=1$.

Figura B.2: A função de autocovariância $(\gamma(u))$ e o espectro de ondaleta $\left(\eta_{j, k}^{(H)}\right)$ com respeito a ondaleta de Haar de um processo MA(1), com $\beta=0,7$ e variância $\sigma^{2}=1$.

Figura B.3: A função de autocovariância $(\gamma(u))$ e o espectro de ondaleta $\left(\eta_{j, k}^{(H)}\right)$ com respeito a ondaleta de Haar de um processo $\mathrm{AR}(1)$, com $\alpha=0,8$ e variância $\sigma^{2}=1$.

Figura B.4: Uma série harmônica $X(t)=\operatorname{sen}\left(\frac{\pi}{12} t\right)+2 \operatorname{sen}\left(\frac{2 \pi}{5} t\right)$.

Figura B.5: $O$ escalograma de $X(t)$ apresentada na Figura B.4 com $T=$ $2^{10}$.

Figura B.6: As transformadas de ondaleta com respeito a ondaleta de Haar de $X(t)$ apresentada na Figura B.4 com $T=2^{10}$.

Figura B.7: O periodograma de ondaleta de $X(t)$ apresentada na Figura B. $4 \operatorname{com} T=2^{10}$.

Figura B.8: Estado de sono de um bebê cuja mãe não consumiu bebidas alcoólicas durande sua gravidez.

Figura B.9: Estado de sono de um bebê cuja mãe consumiu bebidas alcoólicas durante sua gravidez.

Figura B.10: As transformadas de ondaleta com respeito a ondaleta de Haar dos dados unexposed, onde ResolutionLevel $=(M-j)$. 
Figura B.11: As transformadas de ondaleta com respeito a ondaleta de Haar dos dados exposed, onde Resolution Level $=(M-j)$.

Figura B.12: O periodograma de ondaleta dos dados unexposed, onde Resolution Level = $(M-j)$.

Figura B.13: O periodograma de ondaleta dos dados exposed, onde Resolution Level = $(M-j)$.

Figura B.14: O periodograma de ondaleta truncado dos dados unexposed, onde Resolution Level $=(M-j)$.

Figura B.15: O periodograma de ondaleta truncado dos dados exposed, onde ResolutionLevel $=(M-j)$.

Figura B.16: O estado de sono reconstruído dos dados unexposed e exposed.

Figura B.17: O escalograma dos dados unexposed e exposed.

Figura B.18: A imagem do periodograma de ondaleta dos dados unexposed.

Figura B.19: A imagem do periodograma de ondaleta dos dados exposed. 


\section{Apêndice C}

\section{Figuras Citadas no Capítulo 5}

Este apêndice apresenta uma série de figuras citadas no capítulo 5 .

Legenda:

Figura C.1: Produção de carros na Espanha, de janeiro de 1980 a junho de 1996.

Figura C.2: Exportação de carros na Espanha, de janeiro de 1980 a junho de 1996.

Figura C.3: A série ajustada $X(t)$.

Figura C.4: A série ajustada $Y(t)$.

Figura C.5: O periodograma da série $X(t), I_{t, T}^{(x x)}(\lambda)$.

Figura C.6: Parte real do periodograma cruzado entre as séries $X(t)$ e $Y(t)$, $\operatorname{Re} I_{t, T}^{(x y)}(\lambda)$.

Figura C.7: Parte imaginaria do periodograma cruzado entre as séries $X(t)$ e $Y(t), \operatorname{Im} I_{t, T}^{(x y)}(\lambda)$.

Figura C.8: $\mathrm{O}$ espectro estimado da série $X(t), \hat{f}_{x x}(u, \lambda)$.

Figura C.9: Parte real do espectro cruzado estimado entre as séries $X(t)$ e $Y(t), \operatorname{Re} \hat{f}_{x y}(u, \lambda)$.

Figura C.10: Parte imaginária do espectro cruzado estimado entre as séries $X(t)$ e $Y(t), \operatorname{Im} \hat{f}_{x y}(u, \lambda)$.

Figura C.11: A imagem de $\hat{f}_{x x}(u, \lambda)$.

Figura C.12: A imagem de $\operatorname{Re} \hat{f}_{x y}(u, \lambda)$.

Figura C.13: A imagem de $\operatorname{Im} \hat{f}_{x y}(u, \lambda)$.

Figura C.14: Os coeficientes estimados do filtro $a_{u}(v)$. 
Figura C.15: A imagem dos coeficientes estimados do filtro $a_{u}(v)$. 

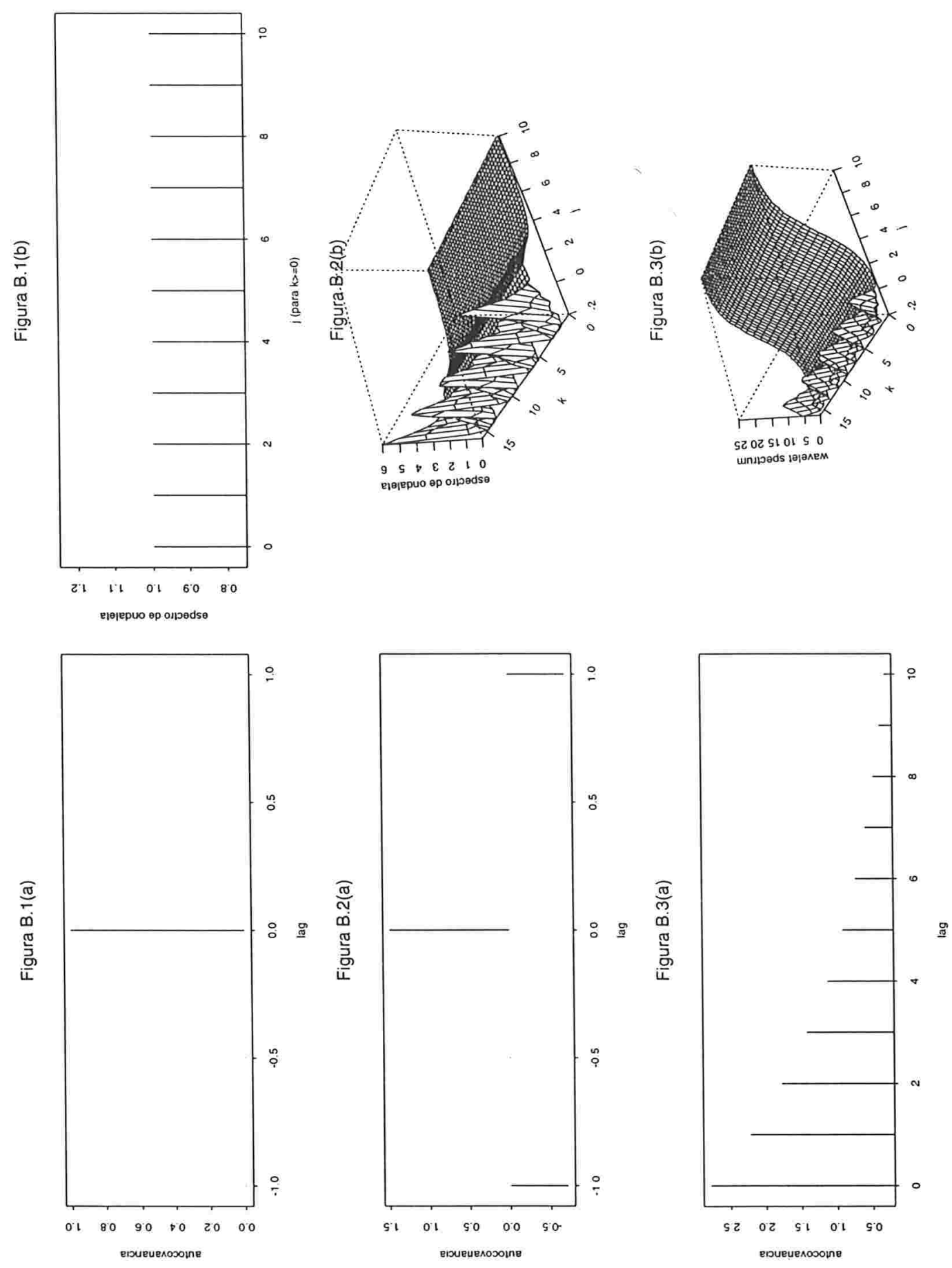
Figura B.4

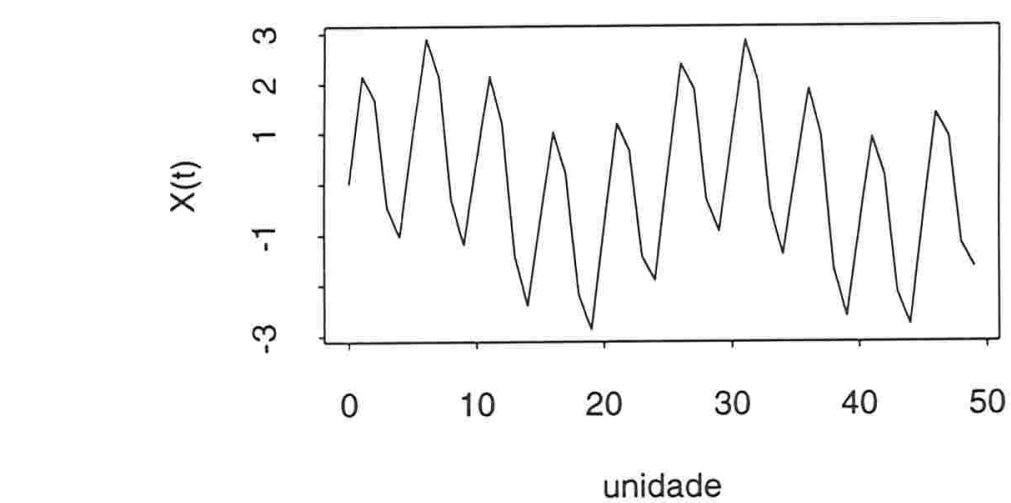

홍

Figura B.6

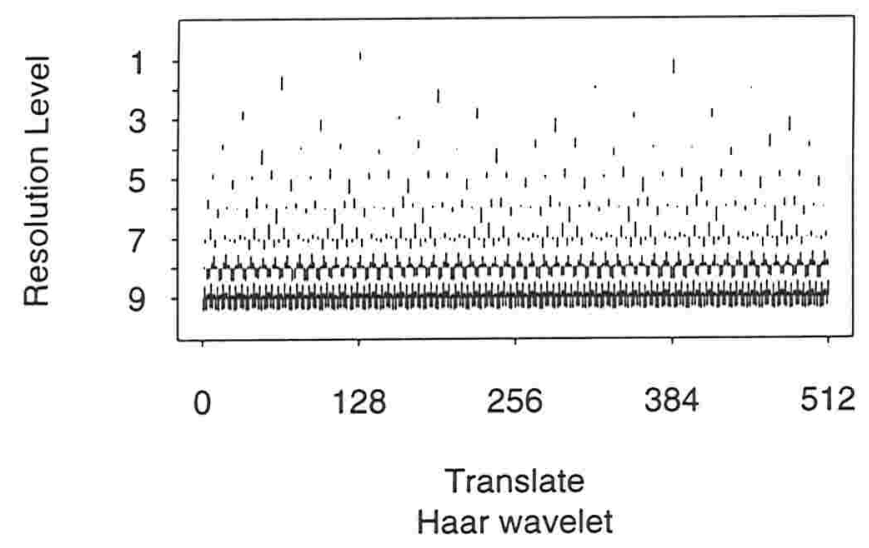

Figura B.5

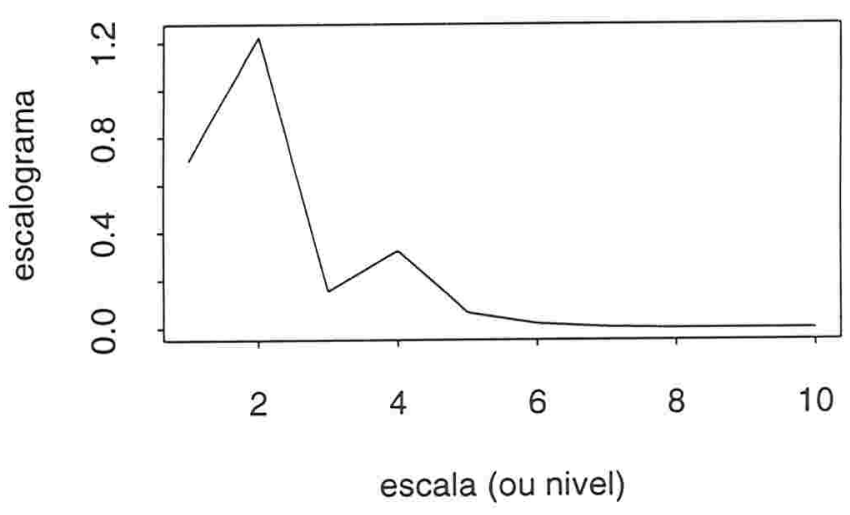

Figura B.7

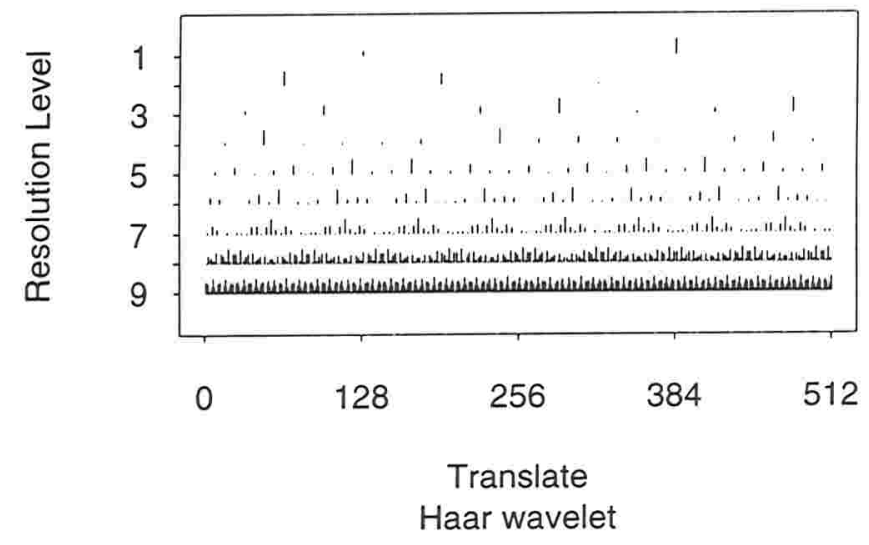


Figura B.8

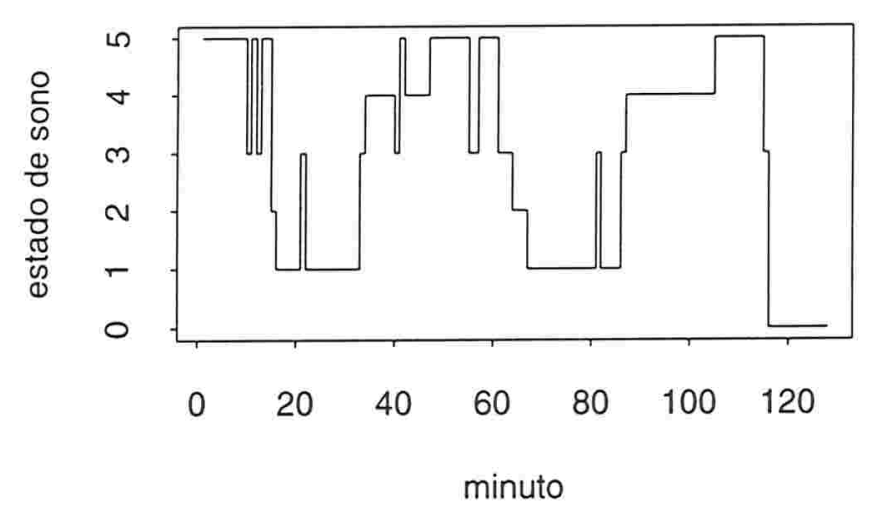

$\underset{0}{-1}$

Figura B.10

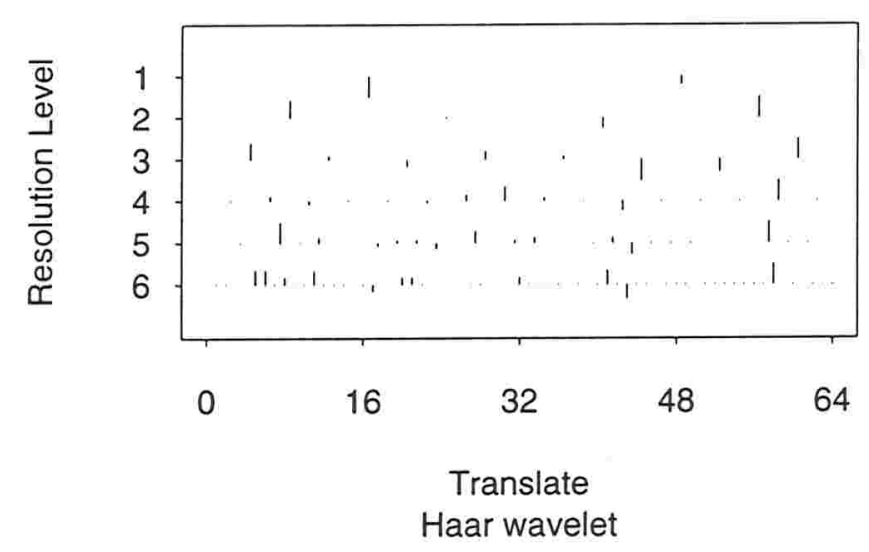

Figura B.9

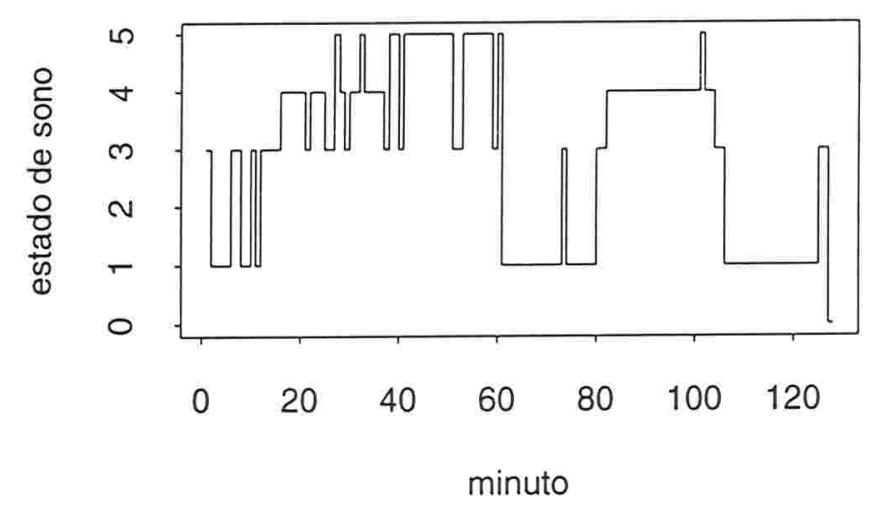

Figura B.11

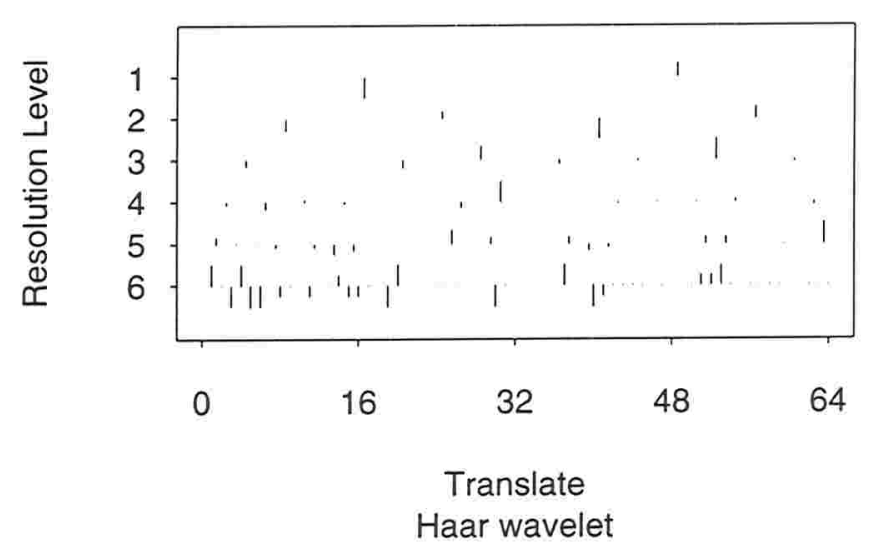


Figura B.12

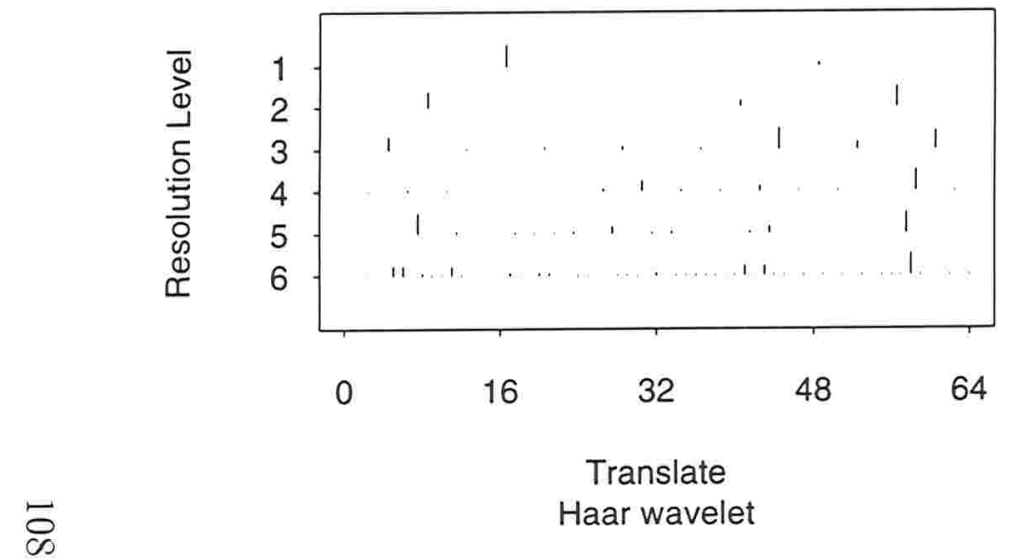

Figura B.14

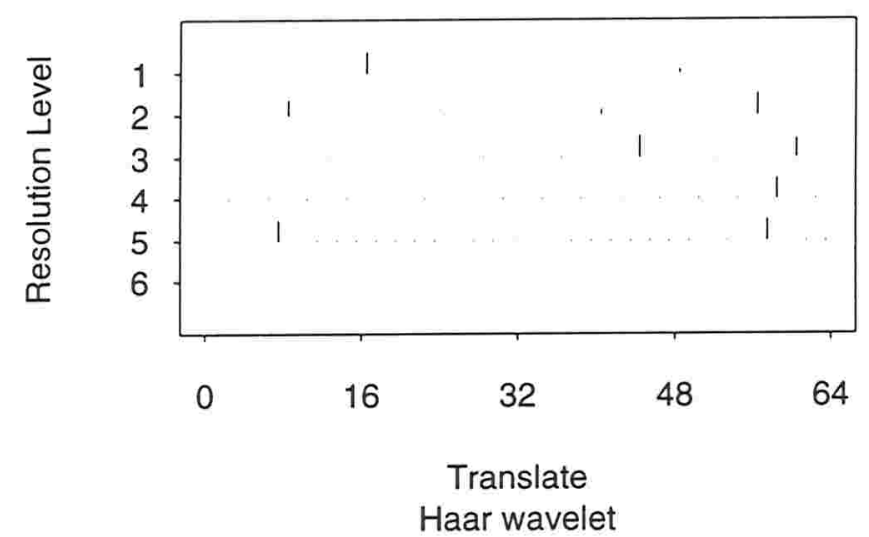

Figura B.13

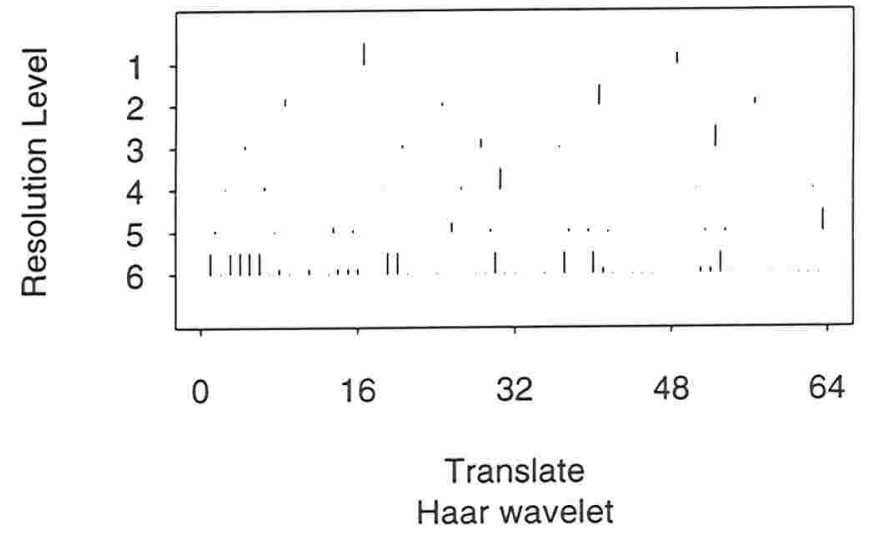

Figura B.15

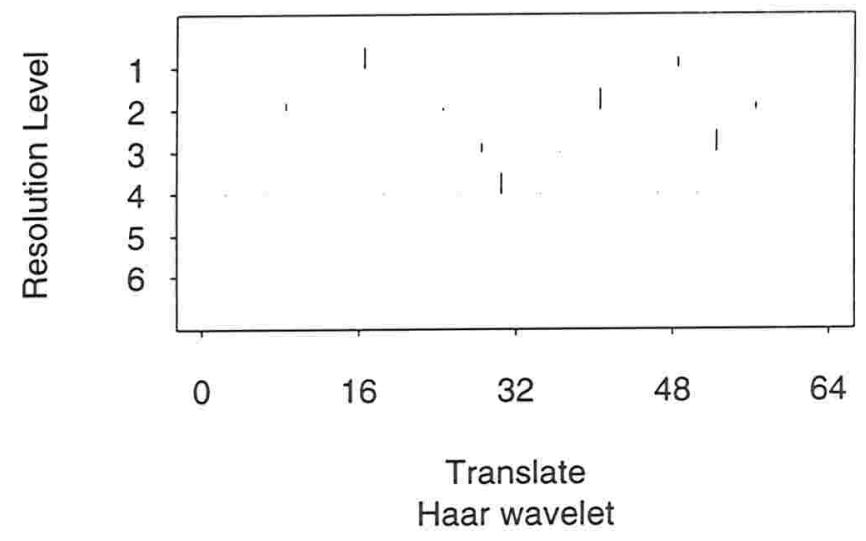



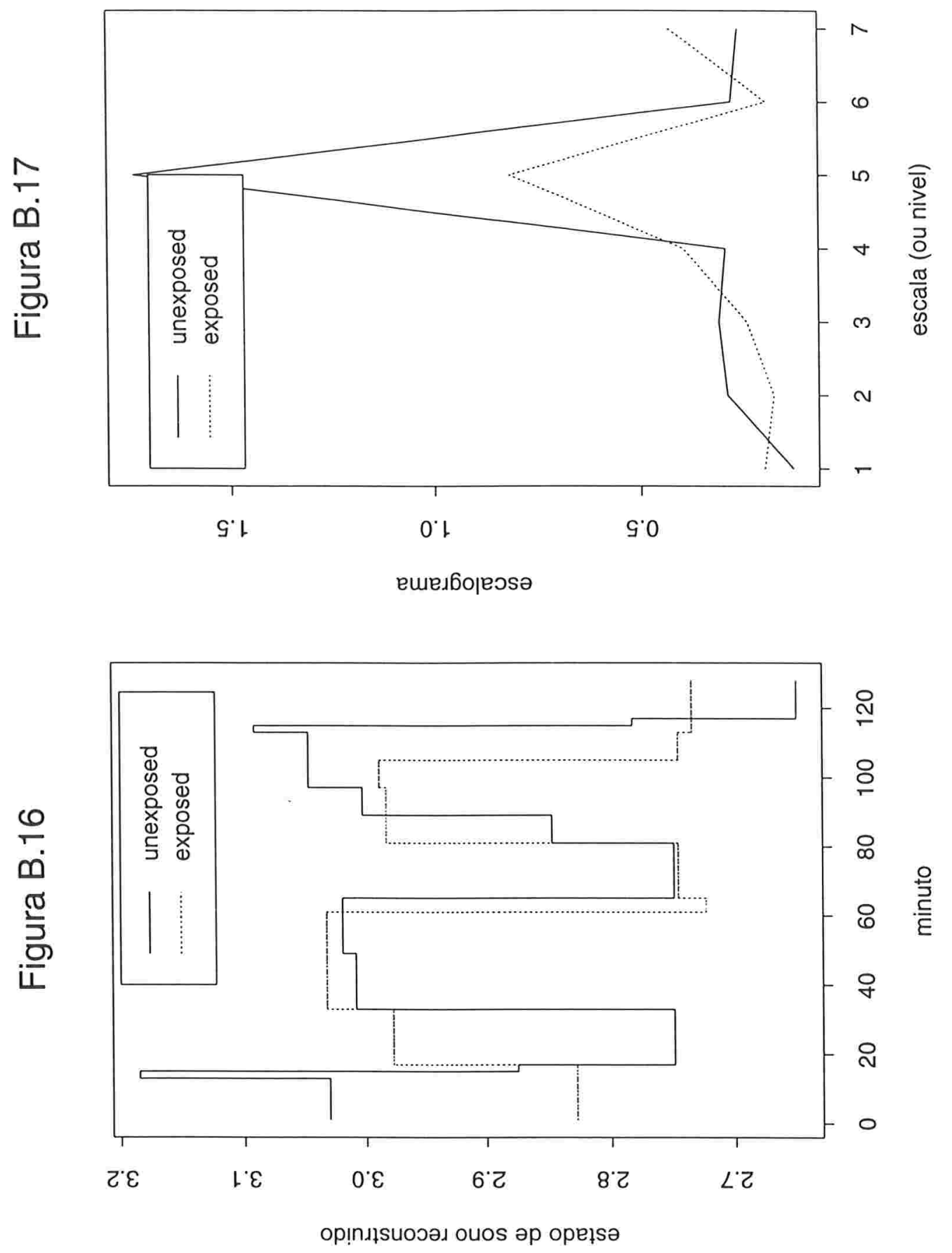

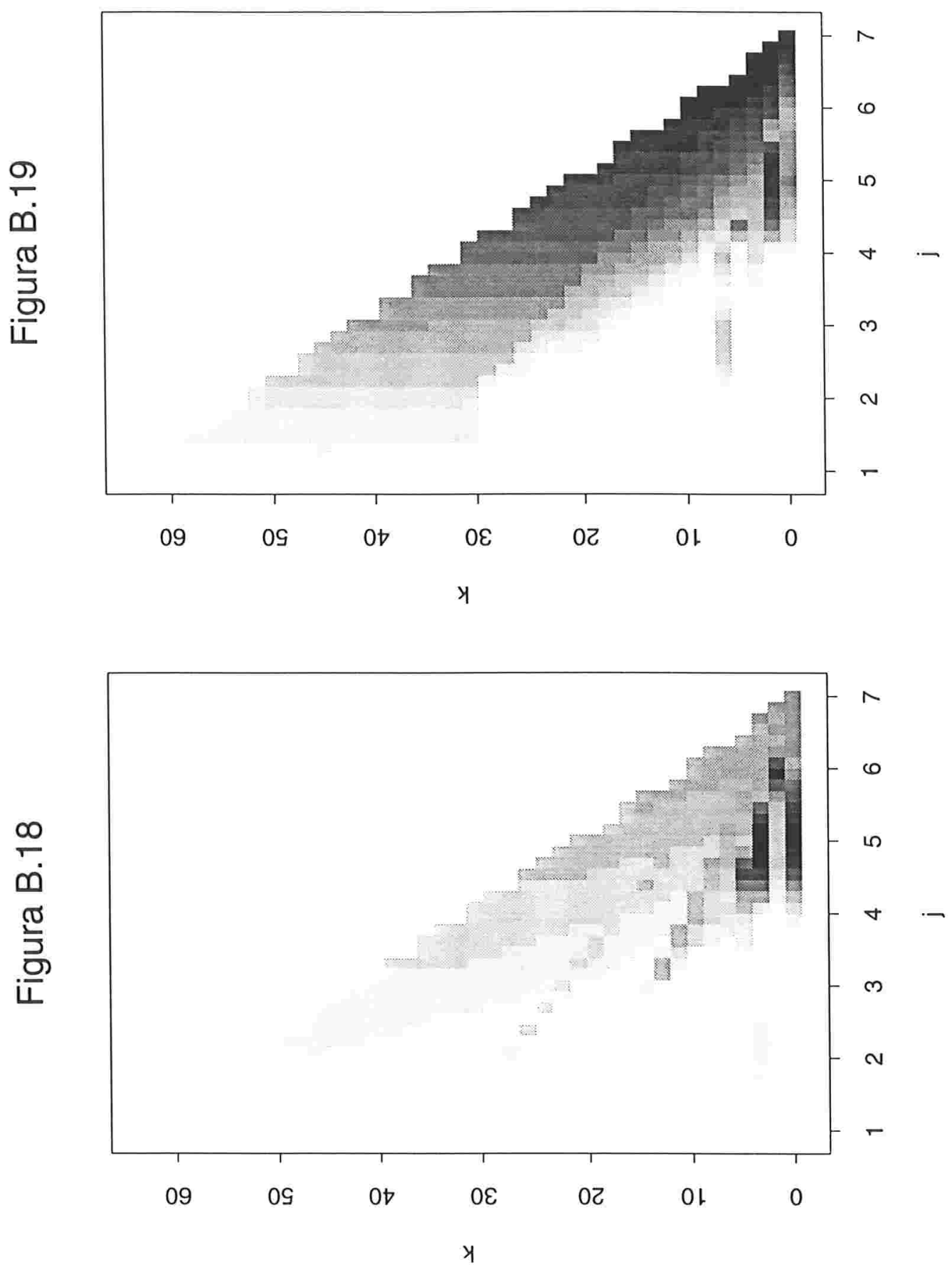


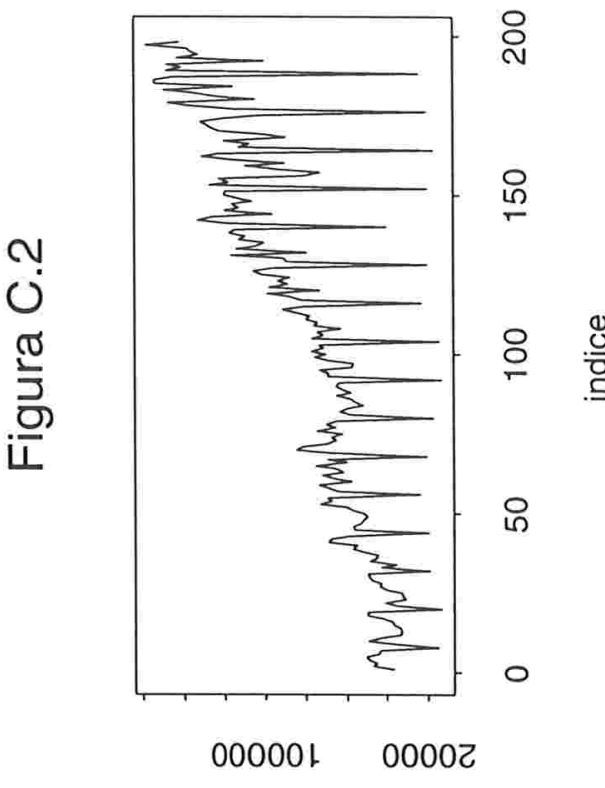

одео әр оеэеноdхә

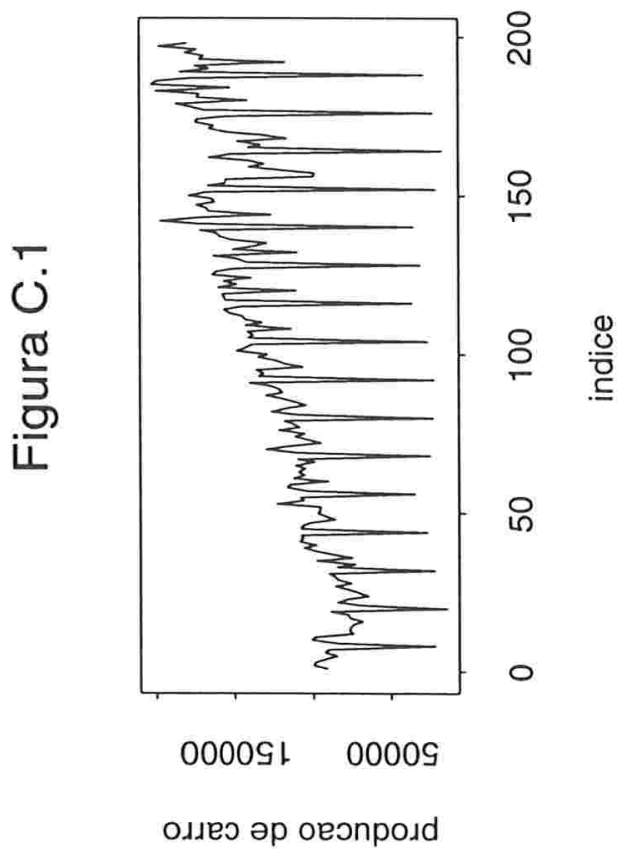

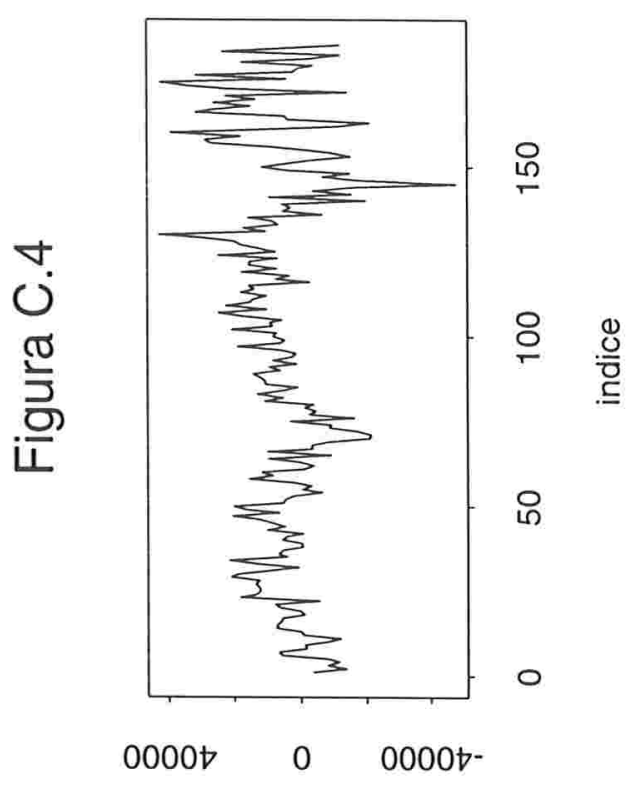

(epeisn!̣) oגеอ әр оеэеноdxә

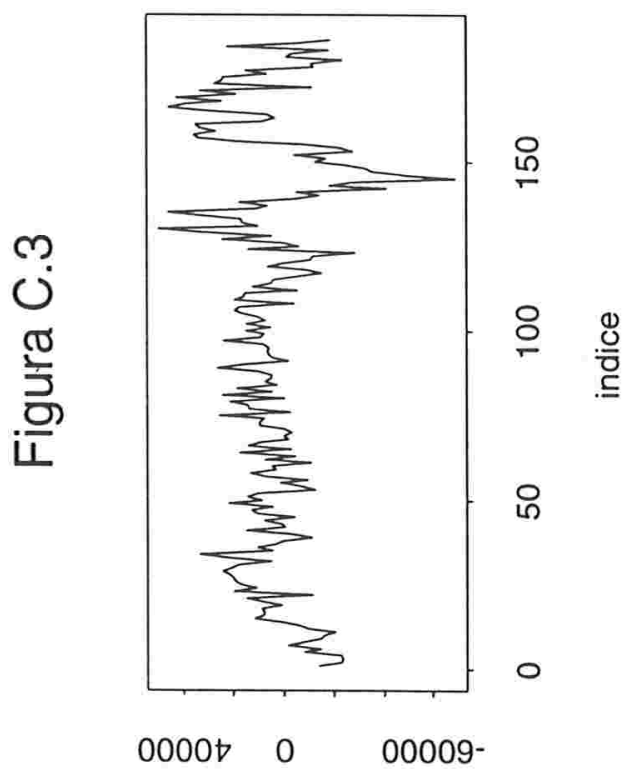

(epełsn(e) oגıeo әp oeonpold 


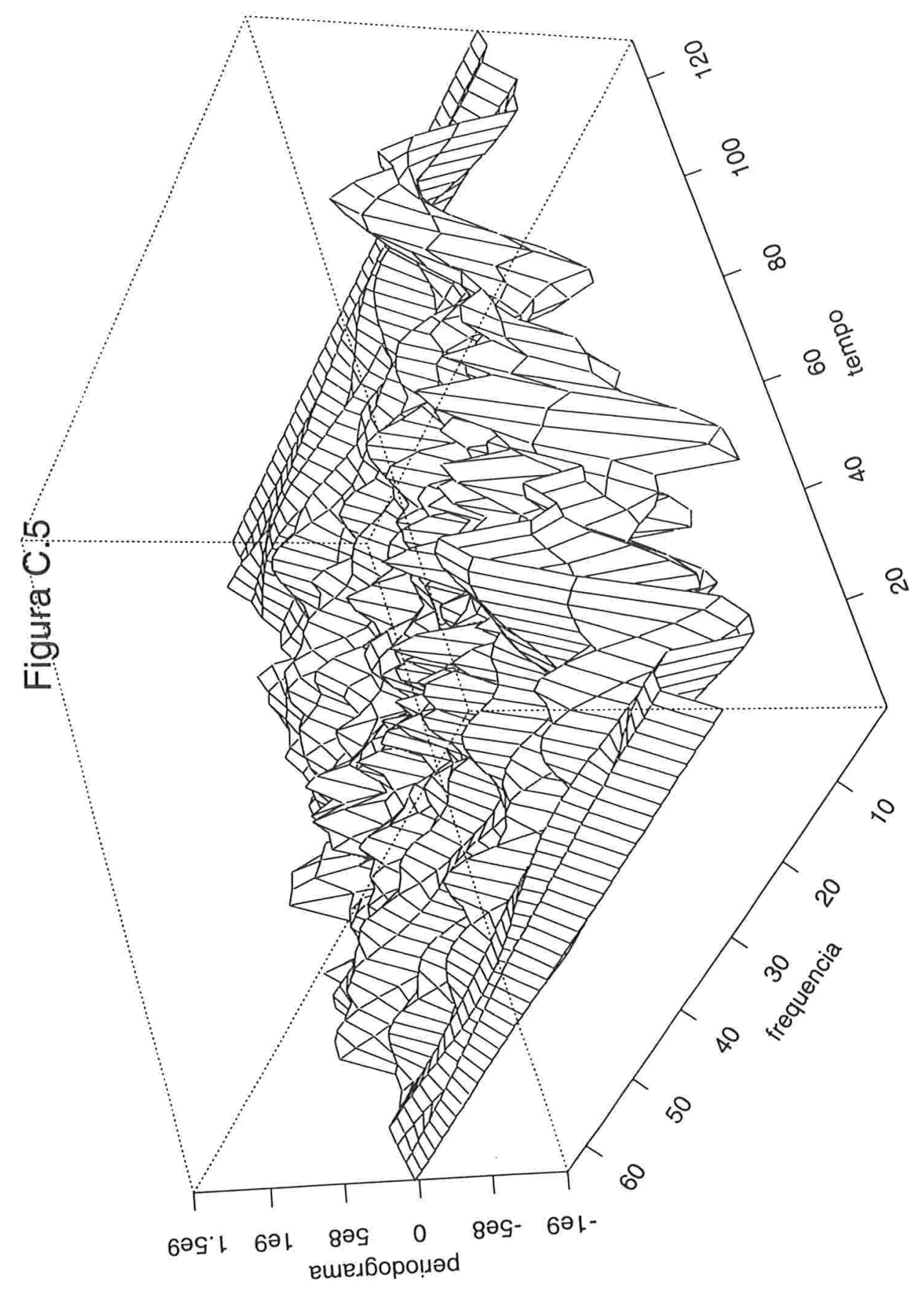




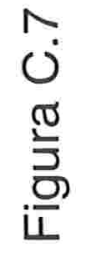

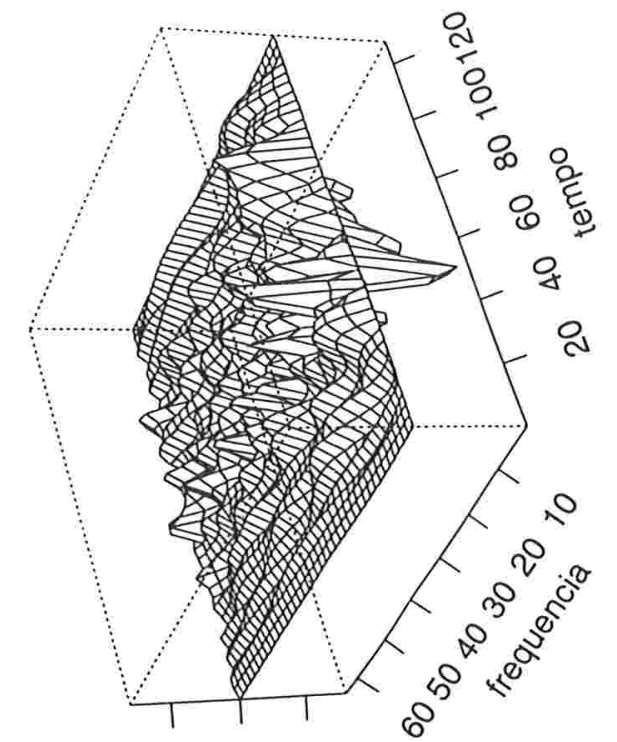

8290 8ə9-

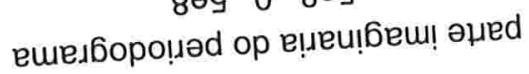

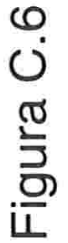

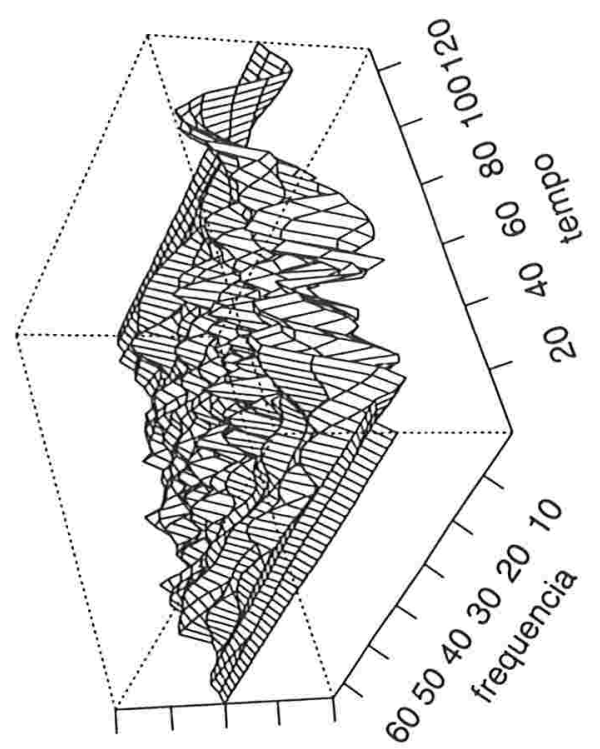

6əเ8ว9 0 8ә96วเ-

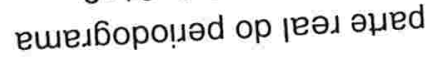




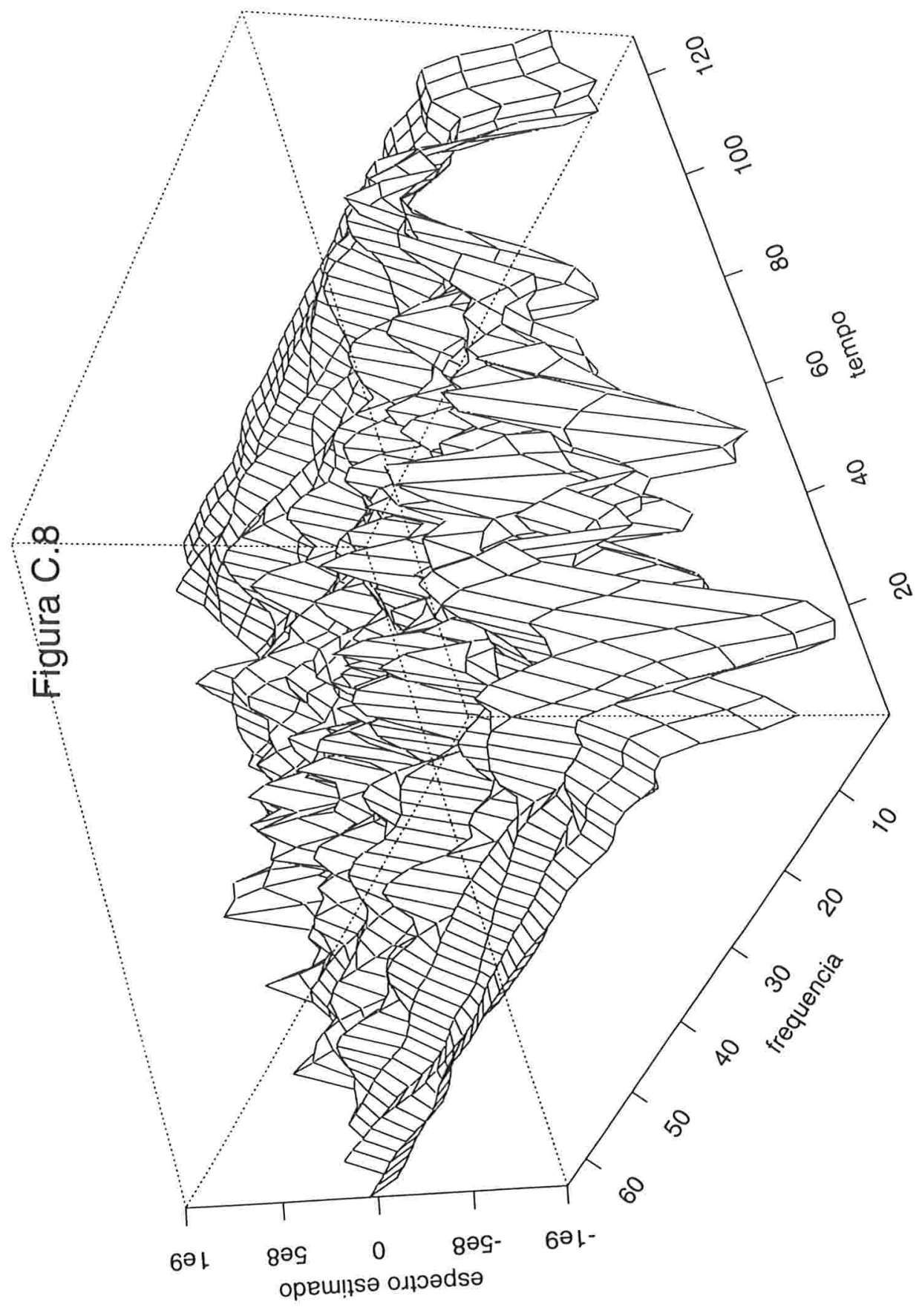




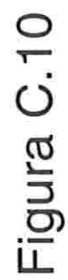

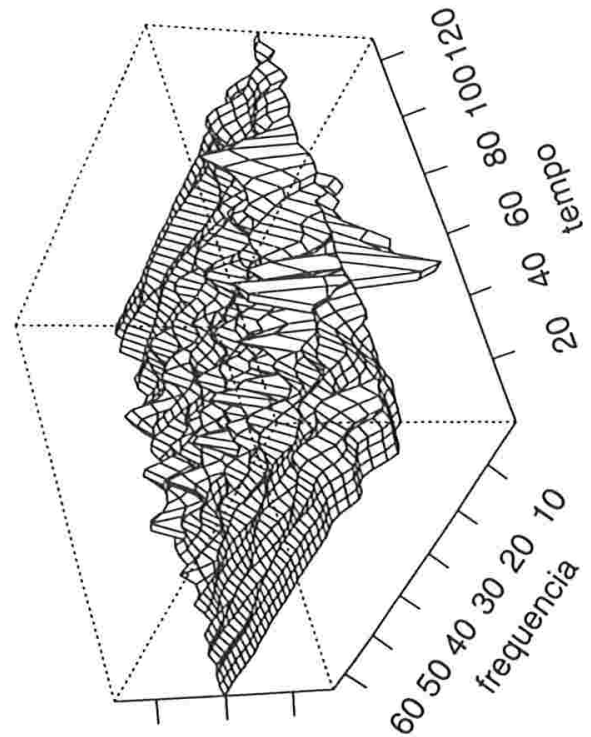

8ə9 0 8ә9-

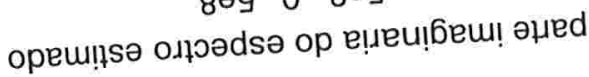

త
뜩
므

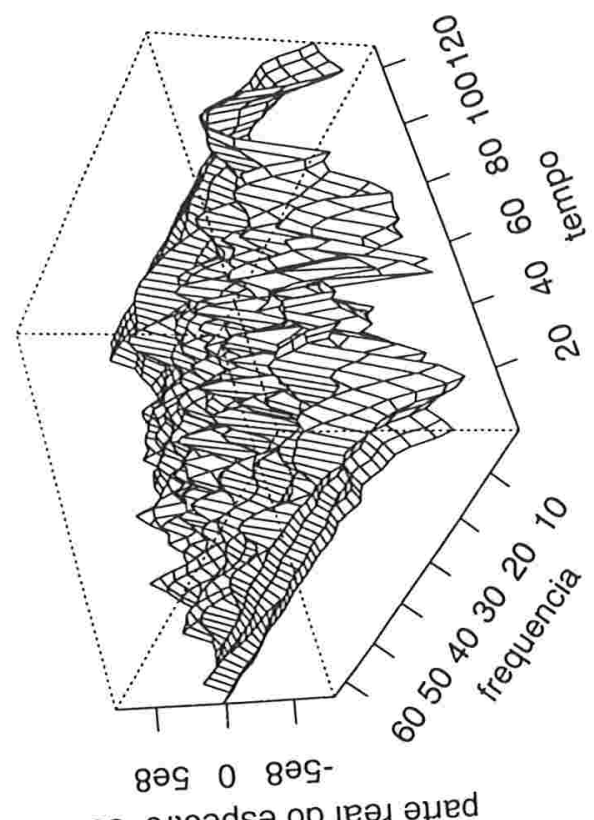

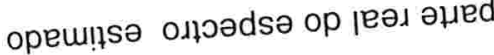



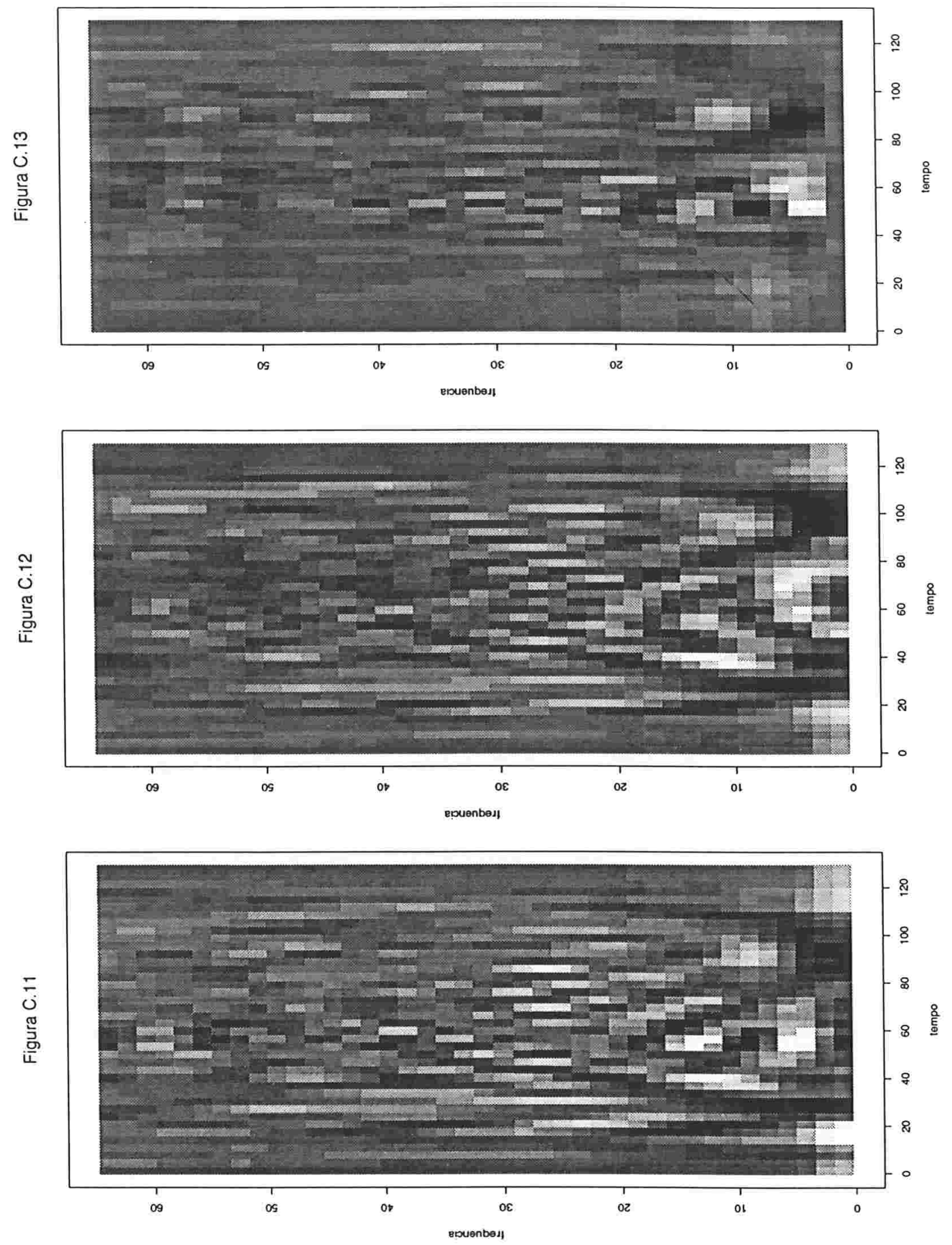

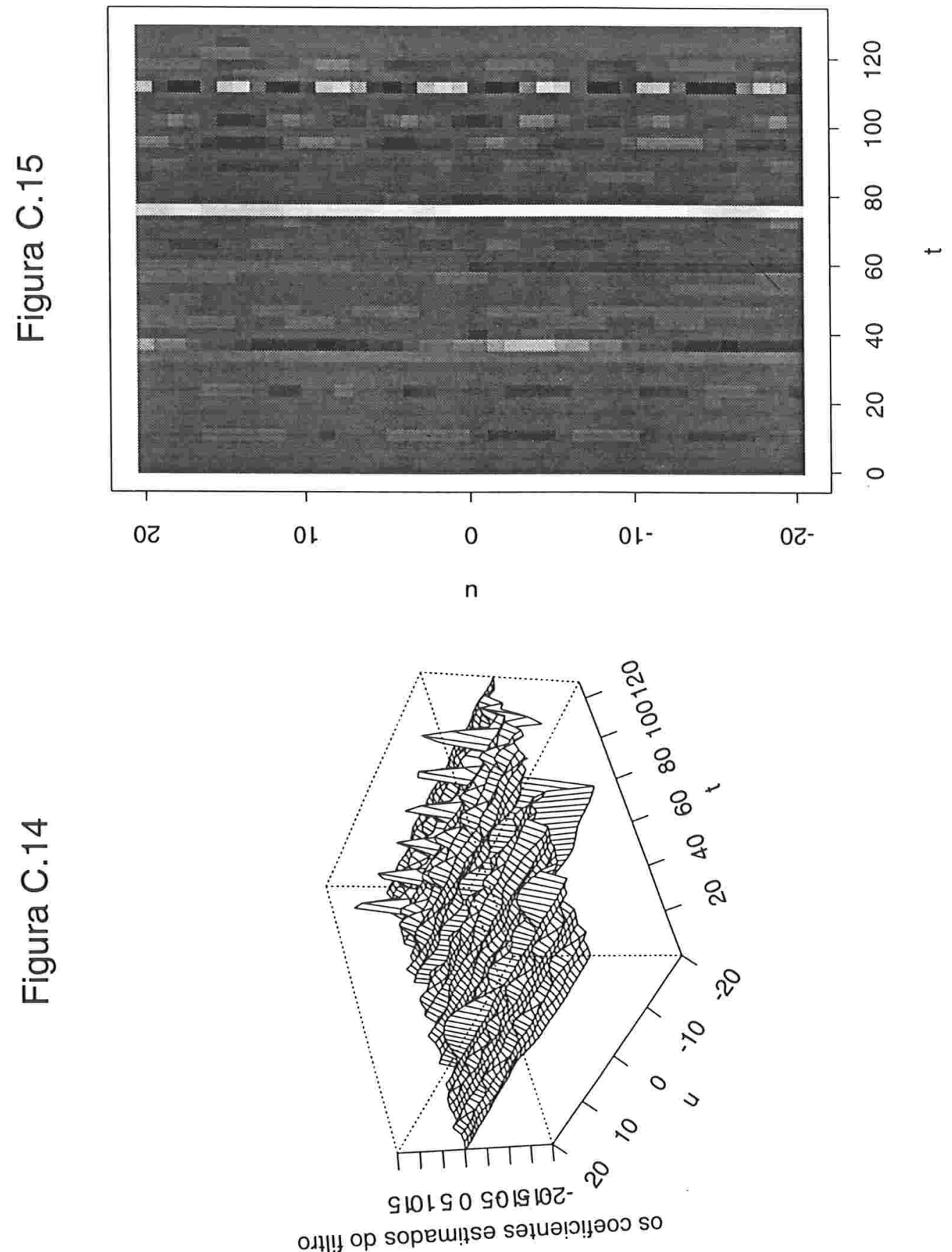